\title{
WestVirginiaUniversity
}

THE RESEARCH REPOSITORY @ WVU

Graduate Theses, Dissertations, and Problem Reports

2003

\section{Evaluation of performance graded asphalt binder equipment and testing protocol}

\author{
Michael E. Pumphrey \\ West Virginia University
}

Follow this and additional works at: https://researchrepository.wvu.edu/etd

\section{Recommended Citation}

Pumphrey, Michael E., "Evaluation of performance graded asphalt binder equipment and testing protocol" (2003). Graduate Theses, Dissertations, and Problem Reports. 1396.

https://researchrepository.wvu.edu/etd/1396

This Thesis is protected by copyright and/or related rights. It has been brought to you by the The Research Repository @ WVU with permission from the rights-holder(s). You are free to use this Thesis in any way that is permitted by the copyright and related rights legislation that applies to your use. For other uses you must obtain permission from the rights-holder(s) directly, unless additional rights are indicated by a Creative Commons license in the record and/ or on the work itself. This Thesis has been accepted for inclusion in WVU Graduate Theses, Dissertations, and Problem Reports collection by an authorized administrator of The Research Repository @ WVU. For more information, please contact researchrepository@mail.wvu.edu. 


\title{
Evaluation of Performance Graded Asphalt Binder Equipment and Testing Protocol
}

\author{
By \\ Michael E. Pumphrey \\ Thesis submitted to the College of Engineering and Mineral Resources \\ at West Virginia University \\ in partial fulfillment of the requirements \\ for the degree of \\ Master of Science in Civil Engineering \\ John P. Zaniewski, Ph.D., Chair \\ Ronald W. Eck, Ph.D. \\ Larry E. Banta, Ph.D. \\ Department of Civil and Environmental Engineering \\ Morgantown, West Virginia \\ 2003
}

Keywords: Asphalt, Binder, Superpave, Performance Graded, SHRP 


\section{Abstract \\ Evaluation of Performance Graded Asphalt Binder Equipment and Testing Protocol}

\section{Michael E. Pumphrey}

The testing and grading of asphalt cement has progressively changed to meet the demanding needs for quality hot mix asphalt pavements. From October 1987 through March 1993, the Strategic Highway Research Program (SHRP) conducted a \$50 million research effort to improve asphalt pavement technology. The research led to the development of the Superpave ${ }^{\mathrm{TM}}$ mix design system (Superior Performing Asphalt Pavements) and the Performance Graded (PG) asphalt binder tests and specifications. The Performance Graded asphalt binder specifications were developed to address the shortcomings of the previous asphalt grading systems.

The objective of this research was to critically evaluate the SHRP performance graded testing equipment purchased by the Asphalt Technology Laboratory at West Virginia University: Rolling Thin Film Oven, Pressure Aging Vessel, Rotational Viscometer, Dynamic Shear Rheometer, and the Bending Beam Rheometer. A testing program was established to evaluate the equipment performance and related test methods and to develop a confidence in the testing protocol and equipment performance such that the new equipment could be used for continued research and industry testing. Sources of equipment and operator error were identified and documented. The underlying theories of the equipment and performance graded specifications were consolidated and documented as well. 


\section{Acknowledgements}

I would like to express my gratitude to my advisor, Dr. John Zaniewski. I would not have been able to continue my pursuit of this Masters of Science degree without his commitment and guidance. Thank you for your friendship and encouragement during this research endeavor.

I would like to extend my thanks to Dr. Ronald Eck and Dr. Larry Banta for their review of my work and willingness to serve on my committee.

Special thanks go to my colleagues and friends at SAI Consulting Engineers, Inc. for their support and patience during this research effort.

I want to thank my wife, Jennifer, for her love, support, prayers, and encouragement. I could not have pursued this degree without you. I love you so much!!

To my daughter, Emily...Daddy is finally finished! Can I have a hug and kiss?

I want to thank my faithful parents for always supporting me. I love you both!

Thanks to my wonderful siblings and in-laws for their prayers and encouragement.

And finally, but most importantly, thank you God for your unfailing love, faithfulness, and strength. 


\section{Table of Contents}

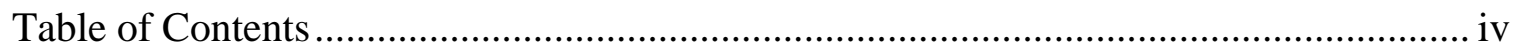

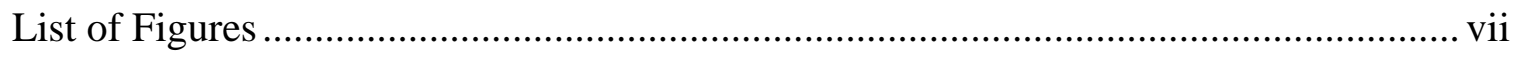

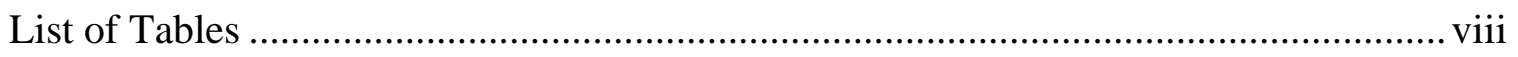

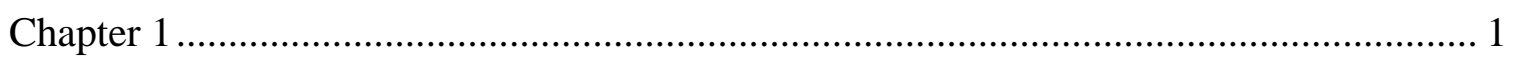

1.1 History of Asphalt Binder Grading Methods ....................................................... 1

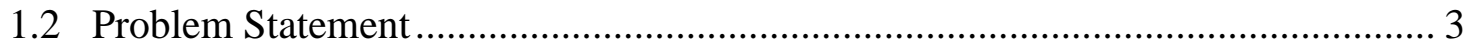

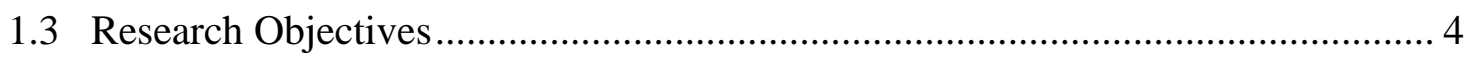

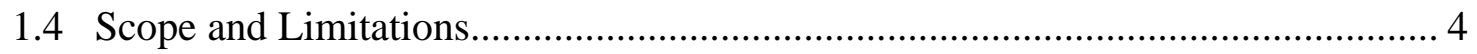

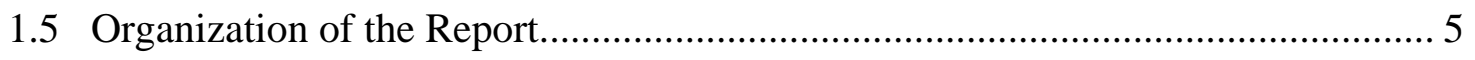

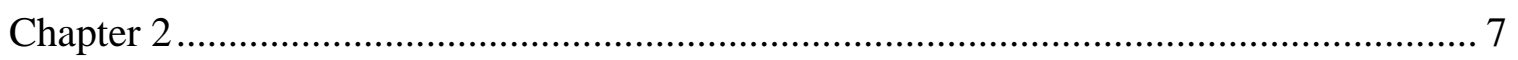

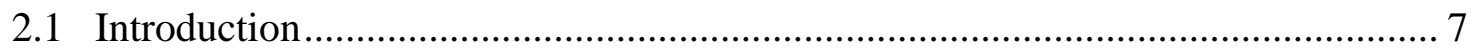

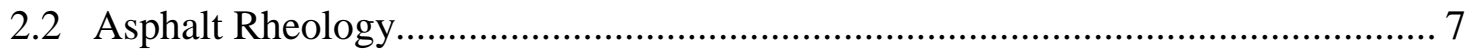

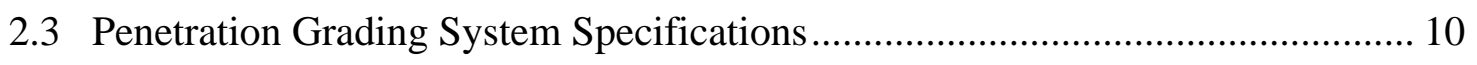

2.4 Viscosity Grading System Specifications ...................................................... 11

2.5 Performance Grading Binder Specifications .............................................. 13

2.6 Rolling Thin Film Oven .......................................................................... 16

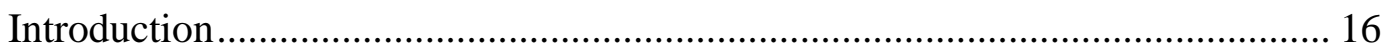

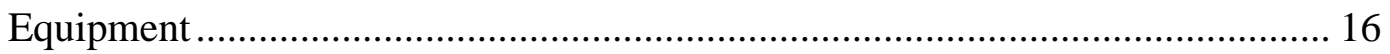

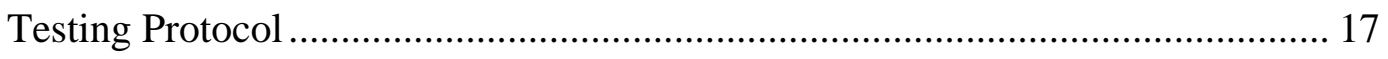

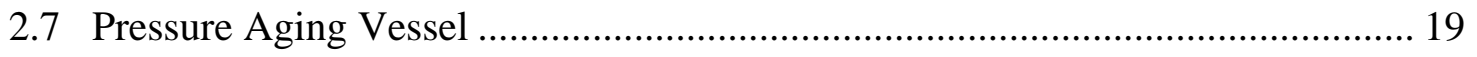

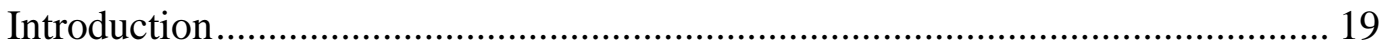

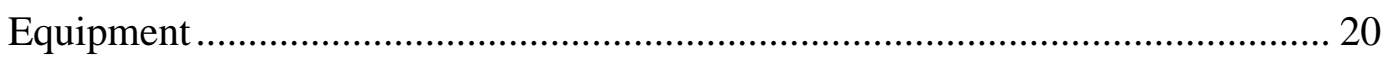

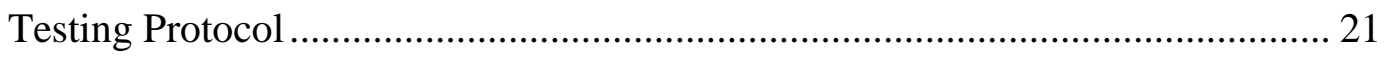

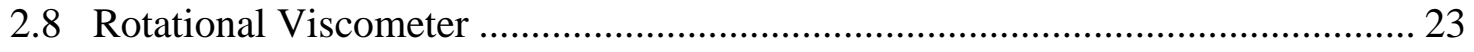

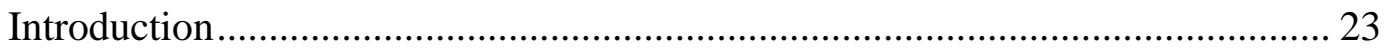

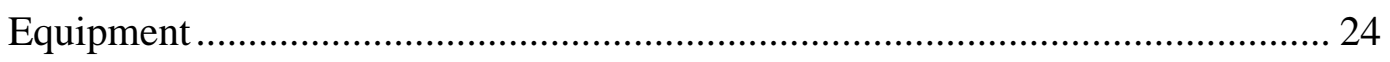

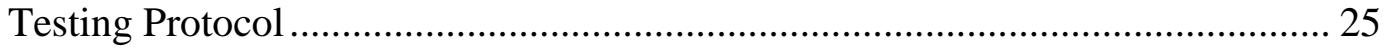

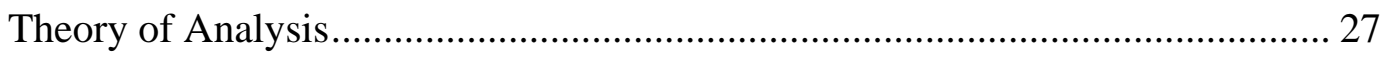

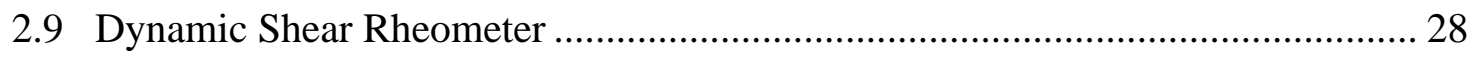




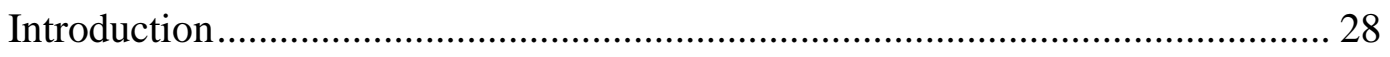

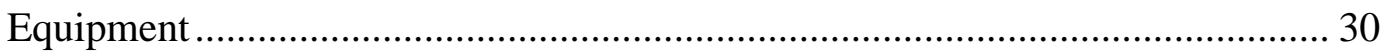

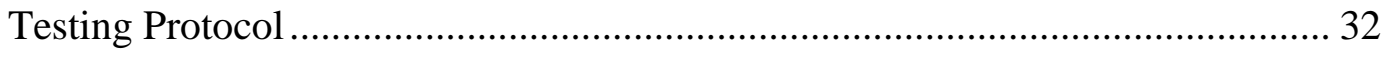

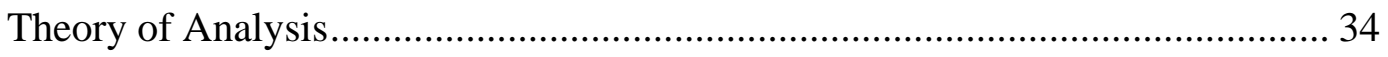

2.10 Bending Beam Rheometer ............................................................................. 46

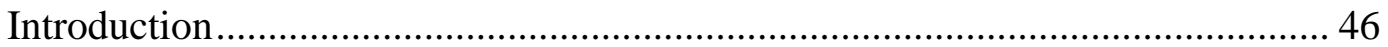

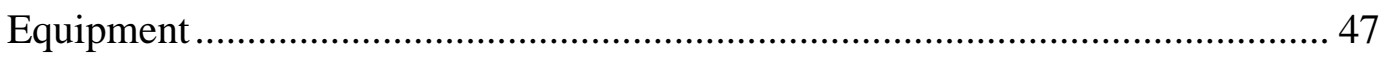

Testing Protocol .......................................................................................... 49

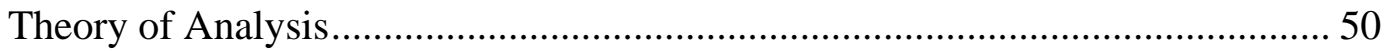

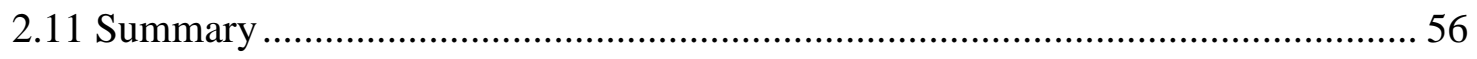

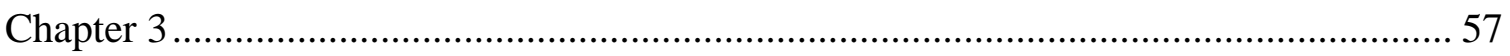

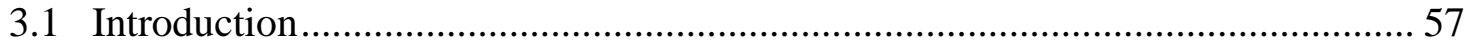

3.2 Testing Program Preparation .................................................................... 57

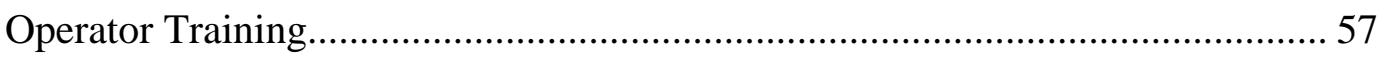

Equipment Preparation.................................................................................... 58

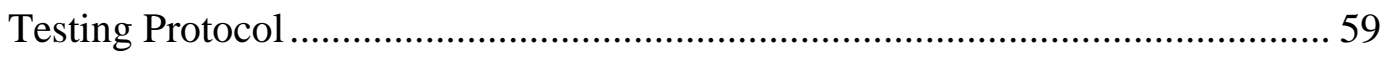

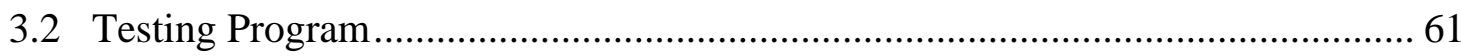

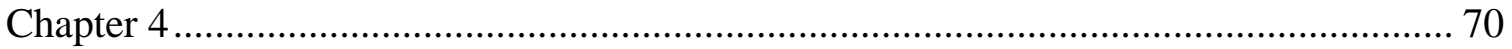

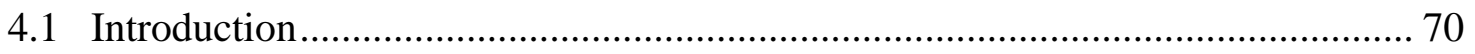

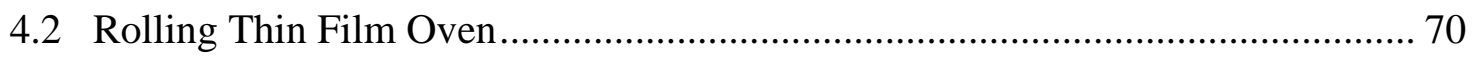

Testing Results Analysis .............................................................................. 70

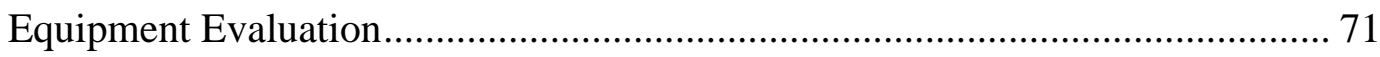

4.3 Pressure Aging Vessel .............................................................................. 72

Testing Results Analysis............................................................................... 72

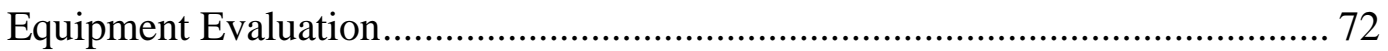

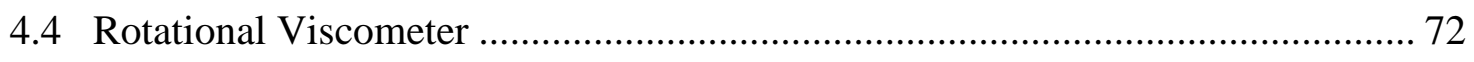

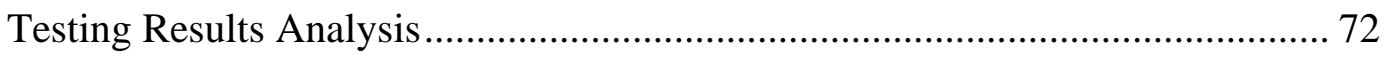

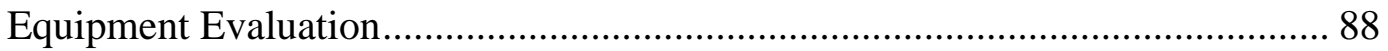

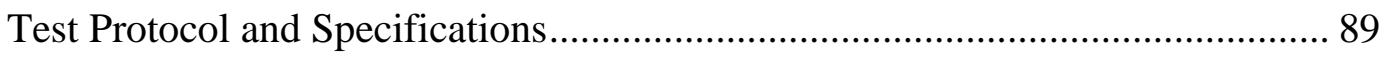

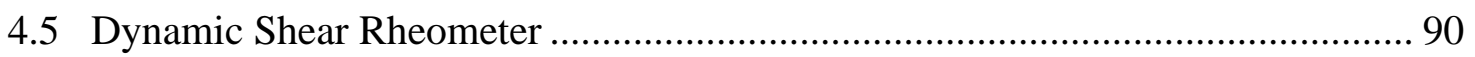

Testing Results Analysis................................................................................. 90 


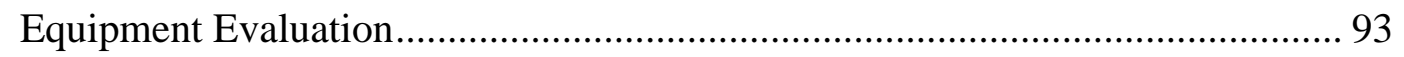

Effect of RTFO Sample Retrieval................................................................. 94

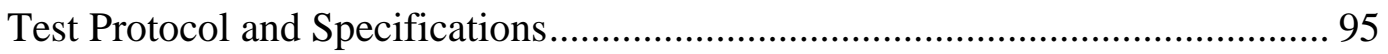

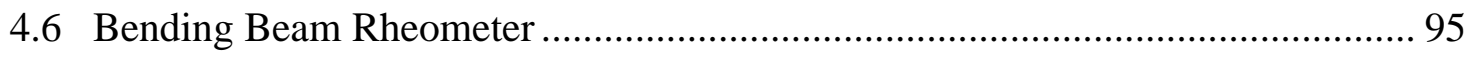

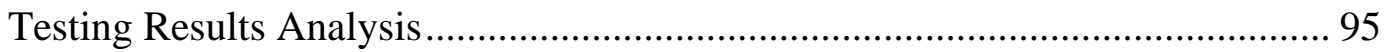

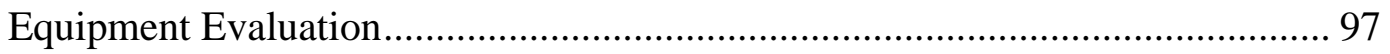

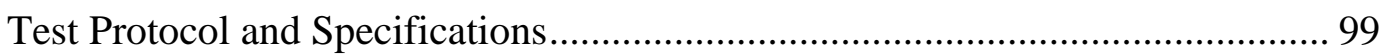

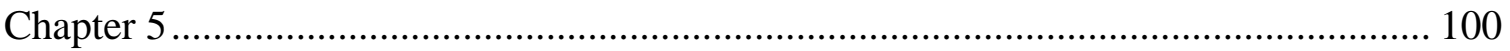

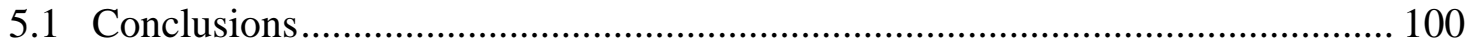

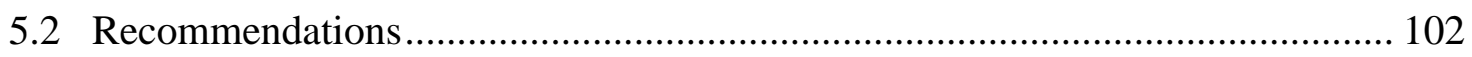

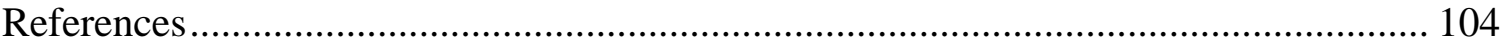

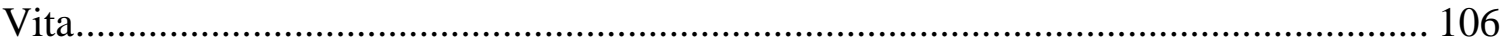




\section{List of Figures}

Figure 2.1 Mechanical Response of Elastic, Viscous, and Viscoelastic Materials........... 8

Figure 2.2 Despatch Rolling Thin Film Oven .......................................................... 17

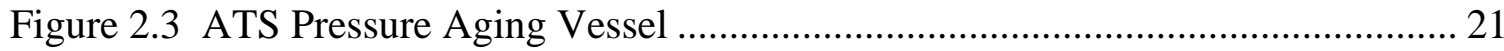

Figure 2.4 Typical Equiviscosity Curve for Asphalt Binder ...................................... 24

Figure 2.5 Brookfield DV-III Rotational Viscometer................................................ 25

Figure 2.6 Bohlin Instruments DSR II Mechanical Dynamic Shear Rheometer ............ 31

Figure 2.7 Configuration and Load Cycle of Dynamic Shear Rheometer..................... 35

Figure 2.8 Phase Angles for Elastic and Viscous Materials ....................................... 38

Figure 2.9 Relationship Between Complex Modulus and Phase Angle ........................ 40

Figure 2.10 Graphical Description of Linear Viscoelastic Region.............................. 41

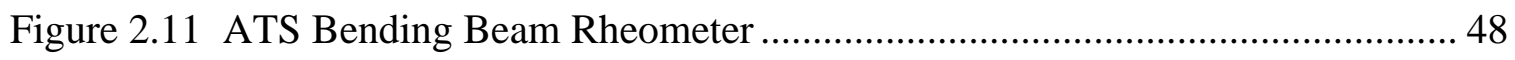

Figure 2.12 Graphical Definition of Creep Rate, m-value........................................... 53

Figure 2.13 Development of Time-Temperature Shift Factors ................................... 54

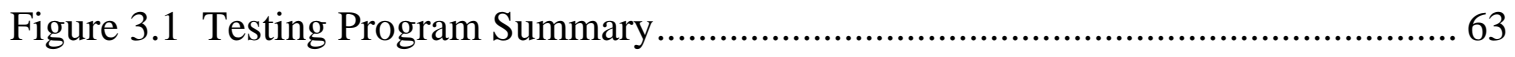

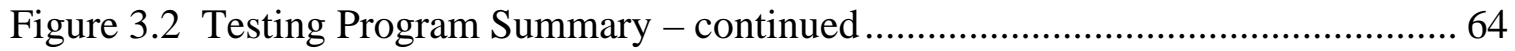

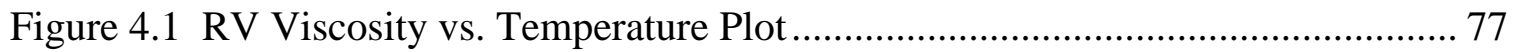

Figure 4.2 RV Test 17 Log-Log Viscosity vs. Log Temperature Plot .......................... 78

Figure 4.3 Viscosity Measured by SHRP Researchers with Capillary and Brookfield

Viscometers, SC4-27 Spindle at 20 RPM............................................... 86

Figure 4.4 RV Temperature Sweep Test 17 Equiviscosity Curve ................................. 87 


\section{List of Tables}

Table 2.1 ASTM D946 Requirements for Penetration Graded Asphalt Cements ........... 10

Table 2.2 ASTM D3381 Requirements for Viscosity Graded Asphalt Cements ............ 12

Table 2.3 Performance Graded Asphalt Cement Grades ............................................ 14

Table 2.4 Summary of AASHTO MP1 Requirements ................................................ 14

Table 2.5 Target Shear Stress and Strain Values ........................................................ 32

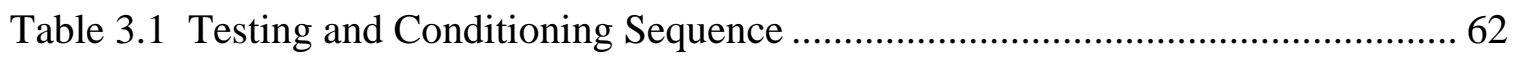

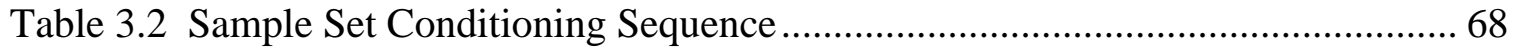

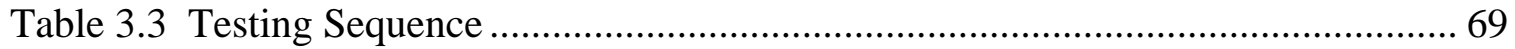

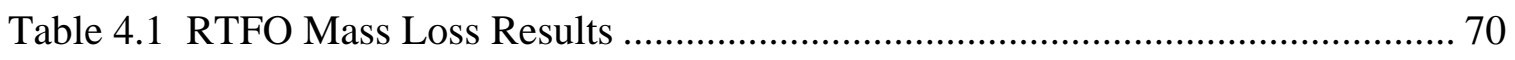

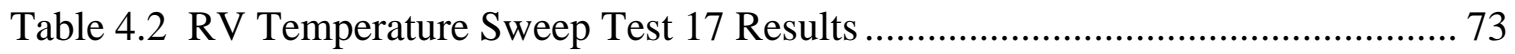

Table 4.3 RV Temperature Sweep Test 18 Results .................................................. 73

Table 4.4 RV Single Temperature Tests 19 through 32 Results ................................... 74

Table 4.5 RV Temperature Sweep Tests Precision Results......................................... 79

Table 4.6 RV Single Temperature Tests Precision Results ........................................ 80

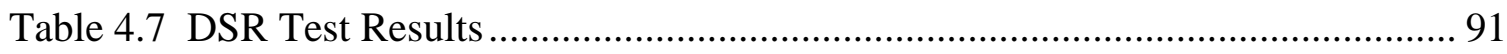

Table 4.8 DSR Tests Precision Results.................................................................... 92

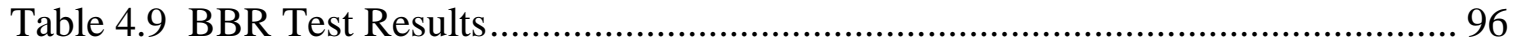

Table 4.10 BBR Tests Precision Results .................................................................... 97 


\section{Chapter 1}

\section{Introduction}

Hot mix asphalt (HMA) pavements are an extensive part of the roadway system in the United States. Building and maintaining HMA highways is an expensive task, therefore, it is vital that a quality product be consistently produced. Efforts are continually being made to increase the quality and performance of the asphalt concrete pavements. Asphalt concrete consists of aggregate and asphalt cement binder. The overall performance of the mixture is largely dependent on the type of asphalt cement binder and its quality. The testing and grading of asphalt cement binders has progressively changed to meet the demanding needs for quality HMA pavements.

\subsection{History of Asphalt Binder Grading Methods}

Roberts, et al. (1996) present a review of asphalt binder grading methods used in the HMA pavement industry, which served as the basis for the following discussion. Prior to 1987, asphalt binders were tested and graded by two primary methods: penetration grading and viscosity grading. The American Association of State Highway Officials (AASHO) published the standard specifications for penetration graded asphalt cements in 1931. The penetration grading system was created to establish different asphalt grades for varying climates and applications. The primary test for penetration grading is the penetration test. The penetration test is an empirical consistency test that measures the penetration of a standard needle into a sample of original asphalt cement at $25{ }^{\circ} \mathrm{C}$ under a standard load and duration. A retained penetration test is also performed on the asphalt binder following short-term aging (hardening) in a thin-film oven. Additional tests are conducted to evaluate the asphalt binder's flash point, purity, and ductility.

The penetration test is purely empirical. It fails to measure the consistency of the binder in fundamental scientific units. In addition, the performance of the binder during testing at $25{ }^{\circ} \mathrm{C}$, which is close to the average pavement service temperature, may not be applicable to the performance of the binder at lower or higher service temperatures. 
The American Association of State and Highway Transportation Officials (AASHTO), American Society for Testing and Materials (ASTM), Federal Highway Association (FHWA), asphalt industry, and several state highway departments sought to replace the empirical tests of the penetration grading system with scientifically-based viscosity tests. The new specification system would measure the consistency of the asphalt at temperatures approximating the maximum pavement surface temperature. The resulting asphalt cement viscosity grading system was initiated in the early 1960's and soon became the most widely used grading system in the United States. The asphalt cement viscosity grading system, also referred to as AC viscosity grading system, characterizes asphalt consistency based on viscosity at $60{ }^{\circ} \mathrm{C}$. Asphalt consistency at near mixing/compacting temperatures is evaluated by conducting viscosity tests at $135{ }^{\circ} \mathrm{C}$. Penetration tests at $25{ }^{\circ} \mathrm{C}$ are performed to evaluate the asphalt's consistency at average service temperatures. Viscosity and ductility tests are performed on thin-film oven aged asphalt samples as well. Additional tests are also conducted to evaluate the asphalt binder's flash point, ductility, and solubility.

A variation of the $\mathrm{AC}$ viscosity grading system is the aged residue viscosity grading system, or AR viscosity grading system. The AR viscosity grading system characterizes asphalt using aged residue from the rolling thin film oven, RTFO. Like the AC viscosity grading system, the consistency of the RTFO-aged asphalt is characterized based on viscosity at $60{ }^{\circ} \mathrm{C}$. Additional specifications include a minimum penetration and minimum viscosity at $25{ }^{\circ} \mathrm{C}$ and $135{ }^{\circ} \mathrm{C}$, respectively. The $\mathrm{AR}$ viscosity grading system has been used primarily by western states.

While the viscosity grading system was an improvement to the penetration grading system, there were still shortcomings. The system still failed to provide a means for evaluating the low temperature performance of asphalt binders. In addition, the system neither evaluated modified asphalt binders nor considered long-term aging of asphalt binders in service. 
From October 1987 through March 1993, the Strategic Highway Research Program (SHRP) conducted a \$50 million research effort to develop performance-based tests and specifications for both asphalt binders and HMA mixtures. The research led to the development of the Superpave ${ }^{\mathrm{TM}}$ mixture design system, which stands for Superior Performing Asphalt Pavements. The Superpave system provides an improved means of asphalt mixture design and analysis.

In addition to the Superpave system, Performance Graded (PG) binder tests and specifications for evaluating asphalt binder properties and performance were also developed during the SHRP research. The new SHRP binder specifications were developed to address the shortcomings of the previous asphalt grading systems. The PG binder tests include four physical tests and two conditioning methods. The conditioning methods are the Rolling Thin Film Oven (RTFO) and the Pressure Aging Vessel (PAV). The physical tests are the Rotational Viscometer (RV), Dynamic Shear Rheometer (DSR), Direct Tension Tester (DTT) and the Bending Beam Rheometer (BBR). The PG binder tests are described in detail in Chapter 2.

\subsection{Problem Statement}

The Asphalt Technology Laboratory at West Virginia University purchased the following SHRP performance graded testing units:

- Despatch Rolling Thin Film Oven

- Applied Test Systems, Inc. (ATS) Pressure Aging Vessel

- NAPCO Model 5831 Vacuum Oven

- Brookfield Model DV-III Rotational Viscometer (RV Series)

- Bohlin Instruments DSR II Mechanical Dynamic Shear Rheometer

- $\quad$ ATS Bending Beam Rheometer

The equipment was new to the Asphalt Technology Laboratory and untried; therefore, an evaluation of the equipment was needed. As with any new testing method and protocol, there was also a learning curve and an adjustment period and that needed to 
be satisfied to develop confidence in the use of the equipment and application of the testing protocol. A confidence in the testing protocol and equipment performance was necessary before the equipment could be used for continued research and industry testing.

\subsection{Research Objectives}

The objective of this research was to critically evaluate the SHRP binder testing equipment in the Asphalt Technology Laboratory at West Virginia University. The research effort was intended to evaluate the equipment calibration, operation and testing procedures. A testing program was established to evaluate the equipment precision and repeatability, as per the applicable AASHTO standard specification precision and bias statements. Equipment problems were to be identified and corrected as necessary. Potential sources of equipment and operator error, if any, were to be identified and documented.

One of the stated advantages of the Performance Graded system is that the tests measure fundamental material characteristics. This implies the test methods are controlled by mechanistic theories. However, the testing methodologies were developed under several research contracts within the Strategic Highway Research Program. Therefore, part of the objective of this research was to consolidate and document the underlying theories of the rotational viscometer, the dynamic shear rheometer, and the bending beam rheometer.

\subsection{Scope and Limitations}

This research was limited to an evaluation of the asphalt binder conditioning and testing equipment available in the Asphalt Technology Laboratory at West Virginia University. The equipment required for the performance grading of asphalt binder is complete except for a direct tension tester, DTT. The final specifications for the direct tension tester were not yet available at the time the West Virginia University Asphalt Technology Laboratory was purchasing the other SHRP testing units. It was not considered prudent to purchase the direct tension tester when the laboratory was established. Therefore, the DTT equipment was not evaluated as part of this research. 
This research was limited to evaluating existing equipment and test methods. There was no effort to develop new equipment or test procedures. The AASHTO Standard Specifications for Transportation Materials and Methods of Sampling and Testing were the sole testing specification used. The AASHTO MP1-97 standard specification was used to evaluate the test results of each unit. The dated AASHTO standard specifications used to conduct the conditioning and testing procedures are as follows:

$$
\begin{aligned}
& \text { Rolling Thin Film Oven - AASHTO T240-97 } \\
& \text { Pressure Aging Vessel - AASHTO PP1-97 } \\
& \text { Rotational Viscometer - AASHTO TP48-97 } \\
& \text { Dynamic Shear Rheometer - AASHTO TP5-98 } \\
& \text { Bending Beam Rheometer - AASHTO TP1-98 }
\end{aligned}
$$

The testing program was conducted using a single operator. All tests were performed with a single grade of asphalt binder, PG 70-22. This is the asphalt grade predominately used for Superpave mixes in West Virginia. The Marathon Ashland Petroleum LLC of Findlay, Ohio was the sole source for the asphalt binder.

Silicone molds were used to prepare the asphalt test specimens for all DSR tests. Aluminum molds were used to prepare the asphalt beam specimens for all the BBR tests. Concern was expressed in reports from the asphalt industry that the BBR silicone molds were producing irregular shaped asphalt beam specimens, thus resulting in the poor test results. Therefore, the decision was made to use the BBR aluminum molds.

\subsection{Organization of the Report}

This thesis is organized into five chapters. Following this introductory chapter is a background literature review. The literature review of Chapter 2 provides background information for each performance grading test and related specifications. The research approach is presented in Chapter 3. The chapter includes a detailed outline of the testing program and methods. The analysis of the test results and the evaluation of the 
equipment performance and testing protocol are provided in Chapter 4. Concluding remarks and recommendations on the research are provided in Chapter 5. 


\section{Chapter 2}

\section{Literature Review and Background}

\section{$2.1 \quad$ Introduction}

Asphalt binders play a critical role in the quality and performance of HMA pavements. Therefore, pavement engineers must have a thorough understanding of asphalt binder behavior. Proven testing equipment and test specifications are necessary to accurately evaluate and select the appropriate asphalt binder for a pavement structure.

This chapter provides a summary of key asphalt rheology concepts that characterize the behavior of asphalt binders. A brief discussion of the penetration grading system and the viscosity grading specifications is presented followed by an overview of the development of the performance grading binder tests and the notable features of the test specifications. The main focus of this chapter is the detailed description of each PG binder test. The description includes the purpose and scope of each test, a summary of method, equipment hardware, test sample and equipment preparation, test procedure summary, unit calibration, and theory of analysis discussion. Some of background information documented in this chapter was developed while setting up and using the equipment and test procedures at the West Virginia University Asphalt Technology Laboratory.

\subsection{Asphalt Rheology}

Asphalt binders deform when subjected to loads. The properties of asphalt also change with varying temperatures. The deformation is a combination of elastic response and viscous flow. The magnitude of deformation, or mechanical response, is dependent on load magnitude, duration, and rate of application and the temperature state of the material. Since asphalt binders display both elastic and viscous response properties, they are classified as viscoelastic materials. Anderson, et al. (1994) present a review of asphalt rheology, which served as the basis for the following discussion. 
The typical elastic, viscous, and viscoelastic responses to an applied stress is shown in Figure 2.1. An elastic material experiences recoverable deformation when subjected to a constant (or creep) load, Figure 2.1a. An elastic material will immediately deform and maintain a constant strain when loaded, Figure 2.1b. The material will immediately return to its initial shape when the creep load is removed. A viscous Newtonian material, when subjected to a constant load, will deform at a constant rate until the load is removed, Figure 2.1c. The deformation of the viscous material, however, will remain after the load is removed; hence, a viscous material experiences nonrecoverable deformation.

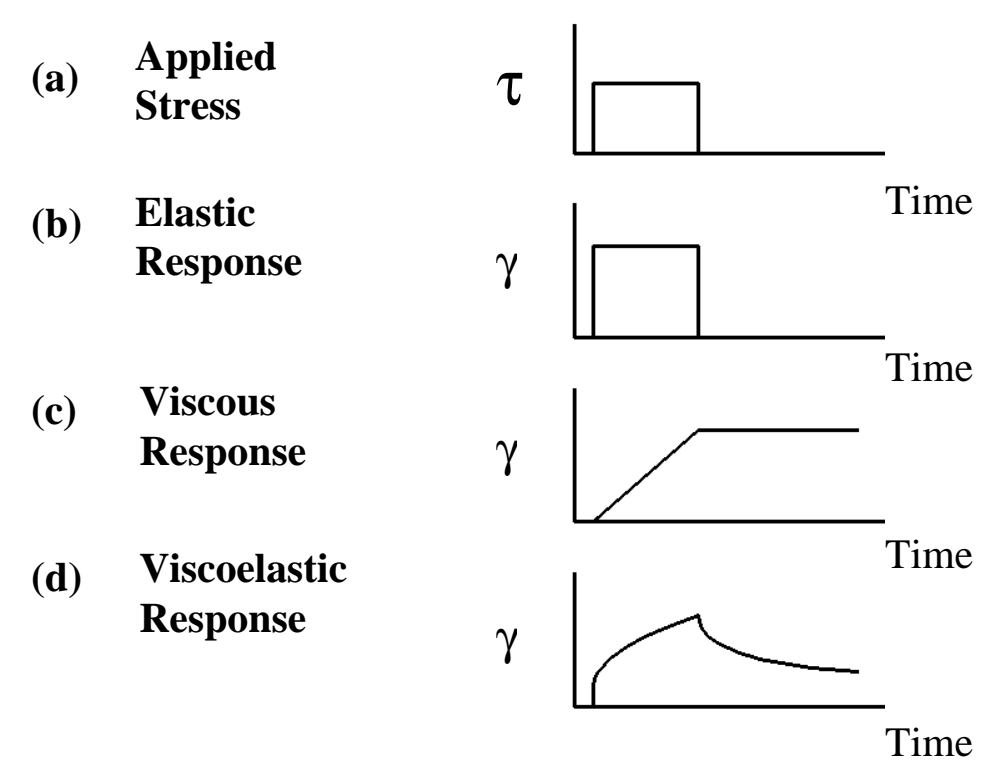

Figure 2.1 Mechanical Response of Elastic, Viscous, and Viscoelastic Materials

A viscoelastic material, when subjected to a creep load, experiences an immediate deformation followed by a continued time-dependent deformation, as shown in Figure 2.1d. The immediate deformation corresponds to the material's elastic response and the time-dependent deformation corresponds to the material's viscous response. Once the load is removed, the viscous deformation component immediately ceases, but this deformation is not recovered. The delayed elastic deformation component is slowly recovered at a decreasing rate. Thus, a viscoelastic material experiences only a partial 
recovery of the deformation resulting from creep loading. The viscoelastic behavior of asphalt can be characterized by its deformation resistance and the relative distribution of that resistance between the elastic component and the viscous component within the linear range. The relative distribution of the resistance between the elastic component and the viscous component is dependent on the asphalt cement characteristics and temperature and loading rate.

The previous loading-response descriptions are for responses within the linear range, which is characterized by the deformation being directly proportional to the applied load at any time and temperature. Nonlinear loading responses are difficult to model for viscoelastic materials such as asphalt. Linear response models, however, are sufficient for the engineering analysis of asphalt binder response to the loading conditions and environmental stresses encountered in the field.

Roberts, et al. (1996) and Bahia and Anderson (1995c) describe the mechanisms that change the rheological properties of asphalt over time. The most familiar of these is age hardening, also referred to as oxidative aging. Asphalt binders consist of hydrocarbons that tend to oxidize when exposed to oxygen. The binder also undergoes volatilization when exposed to the environment. Oxidation and volatilization increases the binder's stiffness (hardness) and viscosity. The time of exposure to the environment (i.e. aging), as well as the service temperatures, directly affect the rate and extent of age hardening.

The greatest extent of age hardening occurs during the production of hot mix asphalt. During the mixing process, the thin film of asphalt binder covering the aggregate is subjected to air temperatures up to $163{ }^{\circ} \mathrm{C}$. The extreme production environment accelerates asphalt binder oxidation and volatilization. Age hardening continues, at a slower rate, during the transportation to the project site and during construction. Thereafter, the rate of age hardening significantly reduces such that extended periods are required to identify changes in asphalt rheological properties. 


\subsection{Penetration Grading System Specifications}

There are five standard penetration grades of asphalt cement used for paving operations, as per ASTM D946. These grades include 40-50, 60-70, 85-100, 120-150, and 200-300. The grades are based on penetration units, where one penetration unit equals one tenth of a millimeter penetration. The lower the penetration grade, the "harder" the asphalt. Therefore, 40-50 grade is the hardest asphalt and 200-300 is the softest. The grades 60-70 and 85-100 are commonly used in the United States. The penetration graded binder specifications are provided in Table 2.1.

\begin{tabular}{|c|c|c|c|c|c|c|c|c|c|c|}
\hline & \multicolumn{10}{|c|}{ Penetration Grade } \\
\hline & 40 & 50 & 60 & 70 & 85 & 100 & 120 & 150 & 200 & 300 \\
\hline & Min & Max & Min & Max & Min & Max & Min & Max & Min & Max \\
\hline $\begin{array}{l}\text { Penetration at } 77^{\circ} \mathrm{F}\left(25^{\circ} \mathrm{C}\right) \text {, } \\
100 \mathrm{~g}, 5 \mathrm{~s}\end{array}$ & 40 & 50 & 60 & 70 & 85 & 100 & 120 & 150 & 200 & 300 \\
\hline $\begin{array}{l}\text { Flash point, }{ }^{\circ} \mathrm{F} \\
\text { (Cleveland open cup) }\end{array}$ & 450 & - & 450 & - & 450 & - & 425 & - & 350 & - \\
\hline $\begin{array}{l}\text { Ductility at } 77^{\circ} \mathrm{F} \\
\left(25^{\circ} \mathrm{C}\right), 5 \mathrm{~cm} / \mathrm{min}, \mathrm{cm}\end{array}$ & 100 & - & 100 & - & 100 & - & 100 & - & 100 & - \\
\hline $\begin{array}{l}\text { Solubility in } \\
\text { trichloroethylene, \% }\end{array}$ & 99.0 & - & 99.0 & - & 99.0 & - & 99.0 & - & 99.0 & - \\
\hline $\begin{array}{l}\text { Retained penetration after } \\
\text { thin-film oven test, } \%\end{array}$ & $55+$ & - & $52+$ & - & $47+$ & - & $42+$ & - & $37+$ & - \\
\hline $\begin{array}{l}\text { Ductility at } 77^{\circ} \mathrm{F}\left(25^{\circ} \mathrm{C}\right) \text {, } \\
5 \mathrm{~cm} / \mathrm{min}, \text { after thin-film } \\
\text { oven test, } \mathrm{cm}\end{array}$ & - & - & 50 & - & 75 & - & 100 & - & $100_{+}$ & - \\
\hline
\end{tabular}

Table 2.1 ASTM D946 Requirements for Penetration Graded Asphalt Cements 


\subsection{Viscosity Grading System Specifications}

There are six AC viscosity grades for asphalt binders specified in ASTM D3381:

AC-2.5, AC-5, AC-10, AC-20, AC-30, and AC-40. Poise is the standard unit of viscosity measurement used by the viscosity grading system. Asphalt graded as AC-2.5 has a viscosity of 250 poises at $60{ }^{\circ} \mathrm{C}$. The lower the asphalt grade, the "softer" the asphalt. Thus, AC-20 is "softer" than AC-30. There are five AR viscosity grades for asphalt cement: AR-1000, AR-2000, AR-4000, AR-8000, and AR-16000. An AR-1000 grade represents an RTFO-aged asphalt with a viscosity of 1000 poises at $60{ }^{\circ} \mathrm{C}$.

The ASTM viscosity graded binder specifications are provided in Table 2.2. As presented in ASTM D3381, Table 2.2 actually includes three tables of requirements: Tables 1 and 2 for AC grades and Table 3 for AR grades. Agencies specifying AC graded asphalt cements could implement either Table 1 or 2 into their specifications. 
Table 1 Requirements for Asphalt Cement, Viscosity Graded at $140^{\circ} \mathrm{F}\left(60^{\circ} \mathrm{C}\right)$

Note - Grading based on original asphalt

\begin{tabular}{llllll}
\hline \multicolumn{1}{c}{ Test } & \multicolumn{5}{c}{ Viscosity Grade } \\
\cline { 2 - 6 } & AC-2.5 & \multicolumn{1}{c}{ AC-5 } & \multicolumn{1}{c}{ AC-10 } & \multicolumn{1}{c}{ AC-20 } & \multicolumn{1}{c}{ AC-40 } \\
\hline Viscosity, $140^{\circ} \mathrm{F}\left(60^{\circ} \mathrm{C}\right), \mathrm{P}$ & $250 \pm 50$ & $500 \pm 100$ & $1000 \pm 200$ & $2000 \pm 400$ & $4000 \pm 800$ \\
Viscosity, $275^{\circ} \mathrm{F}\left(135^{\circ} \mathrm{C}\right)$, min, cSt & 80 & 110 & 150 & 210 & 300 \\
Penetration, $77^{\circ} \mathrm{F}\left(25^{\circ} \mathrm{C}\right), 100 \mathrm{~g}, 5 \mathrm{~s}$, min & 200 & 120 & 70 & 40 & 20 \\
Flash point, Cleveland open cup, min, ${ }^{\circ} \mathrm{F}\left({ }^{\circ} \mathrm{C}\right)$ & $325(163)$ & $350(177)$ & $425(219)$ & $450(232)$ & $450(232)$ \\
Solubility in trichloroethylene, min, $\%$ & 99.0 & 99.0 & 99.0 & 99.0 & 99.0 \\
Tests on residue from thin-film oven test: & & & & \\
Viscosity, $140^{\circ} \mathrm{F}\left(60^{\circ} \mathrm{C}\right)$, max, $\mathrm{P}$ & 1250 & 2500 & 5000 & 10000 & 20000 \\
Ductility, $77^{\circ} \mathrm{F}\left(25^{\circ} \mathrm{C}\right), 5 \mathrm{~cm} / \mathrm{min}, \mathrm{min}, \mathrm{cm}$ & $100^{\mathrm{A}}$ & 100 & 50 & 20 & 10 \\
\hline
\end{tabular}

${ }^{A}$ If ductility is less than 100 , material will be accepted if ductility at $60^{\circ} \mathrm{F}\left(15.5^{\circ} \mathrm{C}\right)$ is 100 minimum at a pull rate of $5 \mathrm{~cm} / \mathrm{min}$.

Table 2 Requirements for Asphalt Cement, Viscosity Graded at $140^{\circ} \mathrm{F}\left(60^{\circ} \mathrm{C}\right)$

Note - Grading based on original asphalt

\begin{tabular}{lllllll}
\hline \multicolumn{1}{c}{ Test } & \multicolumn{5}{c}{ Viscosity Grade } \\
\cline { 2 - 7 } & AC-2.5 & \multicolumn{1}{c}{ AC-5 } & \multicolumn{1}{c}{ AC-10 } & \multicolumn{1}{c}{ AC-20 } & AC-30 & AC-40 \\
\hline Viscosity, $140^{\circ} \mathrm{F}\left(60^{\circ} \mathrm{C}\right), \mathrm{P}$ & $250 \pm 50$ & $500 \pm 100$ & $1000 \pm 200$ & $2000 \pm 400$ & $3000 \pm 600$ & $4000 \pm 800$ \\
Viscosity, $275^{\circ} \mathrm{F}\left(135^{\circ} \mathrm{C}\right)$, min, cSt & 125 & 175 & 250 & 300 & 350 & 400 \\
Penetration, $77^{\circ} \mathrm{F}\left(25^{\circ} \mathrm{C}\right), 100 \mathrm{~g}, 5 \mathrm{~s}$, min & 220 & 140 & 80 & 60 & 50 & 40 \\
Flash point, Cleveland open cup, min, ${ }^{\circ} \mathrm{F}\left({ }^{\circ} \mathrm{C}\right)$ & $325(163)$ & $350(177)$ & $425(219)$ & $450(232)$ & $450(232)$ & $450(232)$ \\
Solubility in trichloroethylene, min, $\%$ & 99.0 & 99.0 & 99.0 & 99.0 & 99.0 & 99.0 \\
Tests on residue from thin-film oven test: & & & & & & \\
$\quad$ Viscosity, $140^{\circ} \mathrm{F}\left(60^{\circ} \mathrm{C}\right)$, max, $\mathrm{P}$ & 1250 & 2500 & 5000 & 10000 & 15000 & 20000 \\
Ductility, $77^{\circ} \mathrm{F}\left(25^{\circ} \mathrm{C}\right), 5 \mathrm{~cm} / \mathrm{min}, \mathrm{min}, \mathrm{cm}$ & $100^{\mathrm{A}}$ & 100 & 75 & 50 & 40 & 25 \\
\hline
\end{tabular}

${ }^{A}$ If ductility is less than 100 , material will be accepted if ductility at $60^{\circ} \mathrm{F}\left(15.5^{\circ} \mathrm{C}\right)$ is 100 minimum at a pull rate of $5 \mathrm{~cm} / \mathrm{min}$.

Table 3 Requirements for Asphalt Cement, Viscosity Graded at $140^{\circ} \mathrm{F}\left(60^{\circ} \mathrm{C}\right)$

Note - Grading based on residue from rolling thin-film oven test.

\begin{tabular}{llllll}
\hline \multirow{2}{*}{ Test on Residue from Rolling Thin-Film Oven Test: } & \multicolumn{5}{c}{ Viscosity Grade } \\
\cline { 2 - 6 } & AR-1000 & AR-2000 & AR-4000 & \multicolumn{1}{c}{ AR-8000 } & AR-16000 \\
\hline Viscosity, $140^{\circ} \mathrm{F}\left(60^{\circ} \mathrm{C}\right), \mathrm{P}$ & $1000 \pm 250$ & $2000 \pm 500$ & $4000 \pm 1000$ & $8000 \pm 2000$ & $16000 \pm 4000$ \\
Viscosity, $275^{\circ} \mathrm{F}\left(135^{\circ} \mathrm{C}\right)$, min, cSt & 140 & 200 & 275 & 400 & 550 \\
Penetration, $77^{\circ} \mathrm{F}\left(25^{\circ} \mathrm{C}\right), 100 \mathrm{~g}, 5 \mathrm{~s}$, min & 65 & 40 & 25 & 20 & 20 \\
\% of original penetration, $77^{\circ} \mathrm{F}\left(25^{\circ} \mathrm{C}\right)$, min & & 40 & 45 & 50 & 52 \\
Ductility, $77^{\circ} \mathrm{F}\left(25^{\circ} \mathrm{C}\right), 5 \mathrm{~cm} / \mathrm{min}, \mathrm{min}, \mathrm{cm}$ & $100^{\mathrm{B}}$ & $100^{\mathrm{B}}$ & 75 & 75 & 75 \\
Tests on original asphalt: & & & & & \\
$\quad$ Flash point, Cleveland open cup, $\mathrm{min},{ }^{\circ} \mathrm{F}\left({ }^{\circ} \mathrm{C}\right)$ & $400(205)$ & $425(219)$ & $440(227)$ & $450(232)$ & $460(238)$ \\
$\quad$ Solubility in trichloroethylene, min, $\%$ & 99.0 & 99.0 & 99.0 & 99.0 & 99.0 \\
\hline
\end{tabular}

A Thin-film oven may be used but the rolling thin-film oven test shall be the referee method.

${ }^{B}$ If ductility is less than 100 , material will be accepted if ductility at $60^{\circ} \mathrm{F}\left(15.5^{\circ} \mathrm{C}\right)$ is 100 minimum at a pull rate of $5 \mathrm{~cm} / \mathrm{min}$.

Table 2.2 ASTM D3381 Requirements for Viscosity Graded Asphalt Cements 


\subsection{Performance Graded Binder Specifications}

The Performance Grading specifications and associated laboratory procedures were designed to relate the asphalt binder rheological characteristics to pavement performance. The Performance Graded binder tests and specifications possess the following notable features (Roberts, et al., 1996):

1. Engineering principles are used to directly relate measured physical properties to field performance.

2. Asphalt is evaluated at three critical stages of asphalt life via special conditioning procedures. The first stage represents the transporting and handling of original asphalt binder before mixing. The second stage represents the short-term aging following HMA production and construction. The third stage considers the continued long-term aging during the pavement service life.

3. The physical properties remain constant for all PG grades, but the temperature at which the properties must be achieved changes in consideration of the asphalt grade required for the project site climate, traffic loading, and speeds.

4. The complete range of service temperatures at the project site is considered.

5. Tests and specifications are established to reduce high temperature rutting, intermediate temperature fatigue cracking, and low temperature thermal cracking.

6. Tests and specifications are established for modified asphalt binders.

7. The specifications maintain solubility criteria.

A summary of the Performance Graded asphalt binder grades and specifications from AASHTO MP1 are provided in Table 2.3 and Table 2.4, respectively. Each grade is represented by two numeric values representing the temperature rating of the binder in degrees Celsius. For example, a PG 70-22 is rated for a climate having an average 7-day maximum design temperature of $70{ }^{\circ} \mathrm{C}$ and a minimum pavement design temperature of $-22{ }^{\circ} \mathrm{C}$. The upper temperature rating is based upon the highest 7-day running average temperature of the pavement, $5 \mathrm{~cm}$ below the pavement surface. The lower temperature rating is based on the coldest estimated pavement temperature $5 \mathrm{~cm}$ below the pavement surface. 


\begin{tabular}{|c|c|c|}
\hline & $\begin{array}{c}\text { High Temp. } \\
{ }^{\circ} \mathbf{C}\end{array}$ & $\begin{array}{c}\text { Low Temp. } \\
{ }^{2} \mathbf{C}\end{array}$ \\
\hline PG & 46 & $-34,-40,-46$ \\
\hline PG & 52 & $-10,-16,-22,-28,-34,-40,-46$ \\
\hline PG & 58 & $-16,-22,-28,-34,-40$ \\
\hline PG & 64 & $-10,-16,-22,-28,-34,-40$ \\
\hline PG & 70 & $-10,-16,-22,-28,-34,-40$ \\
\hline PG & 76 & $-10,-16,-22,-28,-34,-40$ \\
\hline PG & 82 & $-10,-16,-22,-28,-34,-40$ \\
\hline
\end{tabular}

Notes:

${ }^{1}$ Average 7-day maximum pavement design temperature

${ }^{2}$ Minimum pavement design temperature

\section{Table 2.3 Performance Graded Asphalt Cement Grades}

\begin{tabular}{|c|c|c|c|c|c|}
\hline Test & Performance Parameter & $\begin{array}{l}\text { Asphalt Binder } \\
\text { Condition State }\end{array}$ & Specification & $\begin{array}{c}\text { Specification } \\
\text { Limit }\end{array}$ & $\begin{array}{c}\text { Test Temp. }{ }^{1} \\
{ }^{\circ} \mathrm{C}\end{array}$ \\
\hline $\mathrm{RV}$ & Flowability & Neat & Viscosity & 3 Pa.s (Max.) & 135 \\
\hline DSR & Rutting resistance & Neat & $\mathrm{G}^{*} / \mathrm{sin} \mathrm{d} @ 10 \mathrm{rad} / \mathrm{sec}$. & $1.0 \mathrm{kPa}$ (Min.) & High \\
\hline DSR & Rutting resistance & RTFO-aged & $\mathrm{G}^{*} / \mathrm{sin} \mathrm{d} @ 10 \mathrm{rad} / \mathrm{sec}$. & $2.2 \mathrm{kPa}$ (Min.) & High \\
\hline DSR & $\begin{array}{l}\text { Fatigue cracking } \\
\text { resistance }\end{array}$ & PAV-aged ${ }^{2}$ & $\mathrm{G}^{*} \sin \mathrm{d} @ 10 \mathrm{rad} / \mathrm{sec}$ & $5000 \mathrm{kPa}$ (Max.) & Intermediate \\
\hline \multirow{2}{*}{ BBR } & \multirow{2}{*}{$\begin{array}{l}\text { Thermal cracking } \\
\text { resistance }\end{array}$} & \multirow{2}{*}{ PAV-aged ${ }^{2}$} & $\begin{array}{c}\text { Creep Stiffness, S } \\
\text { @ } 60 \text { sec. }\end{array}$ & $300 \mathrm{MPa}$ & Low $+10^{\circ} \mathrm{C}$ \\
\hline & & & m-value @ 60 sec. & 0.300 & Low $+10^{\circ} \mathrm{C}$ \\
\hline DTT & $\begin{array}{c}\text { Thermal cracking } \\
\text { resistance }\end{array}$ & PAV-aged ${ }^{2}$ & $\begin{array}{c}\text { Failure Strain } \\
@ 1.0 \mathrm{~mm} / \mathrm{min} . \\
\end{array}$ & $1.0 \%$ & Low $+10^{\circ} \mathrm{C}$ \\
\hline
\end{tabular}

Additional Specifications:

Flash Point Temperature $=230{ }^{\circ} \mathrm{C}$ (Min.)

RTFO Mass Loss $=1.0 \%$ (Max.)

Notes:

\footnotetext{
${ }^{1}$ High temperature - see Table 2.3

Low temperature - see Table 2.3

Intermediate temperature $=($ High temp + Low temp. $) / 2+4{ }^{\circ} \mathrm{C}$

${ }^{2}$ Pressure Aging Vessel Conditioning Temperatures per PG Grade:

PG $46-90^{\circ} \mathrm{C} \quad \mathrm{PG} 70-100^{\circ} \mathrm{C}\left(110^{\circ} \mathrm{C}\right.$ for desert climates $)$

PG $52-90^{\circ} \mathrm{C} \quad \mathrm{PG} 76-100^{\circ} \mathrm{C}\left(110^{\circ} \mathrm{C}\right.$ for desert climates $)$

PG $58-100^{\circ} \mathrm{C} \quad \mathrm{PG} 82-100^{\circ} \mathrm{C}\left(110^{\circ} \mathrm{C}\right.$ for desert climates $)$
}

$\mathrm{PG} 64-100^{\circ} \mathrm{C}$

Table 2.4 Summary of AASHTO MP1 Requirements 
The rotational viscometer and dynamic shear rheometer have been used for rheological evaluation in many industries for years (Petersen, et al., 1994a). The rolling thin film oven, which is an improved variation of the thin film oven used in the viscosity grading method, was developed prior to the SHRP study. A comprehensive study of the existing rolling thin film oven was considered as part of the SHRP project. However, since the evaluation of the rolling thin film oven would have consumed too much of the project resources and no long-term field aging specifications existed, the SHRP researchers never initiated the study.

Instead of evaluating the rolling thin film oven, focus was placed on developing a long-term aging procedure. The pressure aging methods used for years in the asphalt research field and the rubber products industries were evaluated for use with the PG binder specifications. The study led to the adoption of the pressure aging vessel test, a modified form of the pressure aging methods from other industries.

Several attempts have been made in the past, with very little success, to develop an inexpensive device for evaluating the low temperature performance of asphalt binders (Bahia and Anderson, 1995b). Prior to the SHRP research, Pennsylvania State University introduced a bending beam rheometer for evaluating low temperature performance. The bending beam rheometer was refined during the SHRP research project and was incorporated into the PG binder specification.

No test methods existed for characterizing the fatigue or the fracture properties of asphalt binders prior to the SHRP research (Petersen, et al., 1994b). The direct tension test was therefore developed to evaluate the tensile failure properties of asphalt binders at low temperatures. The final test specifications for the direct tension tester were not yet available at the time the West Virginia University Asphalt Technology Laboratory was purchasing the other SHRP performance graded testing units. 


\subsection{Rolling Thin Film Oven}

\section{Introduction}

\section{Purpose and Scope}

Significant age hardening occurs during the production and construction of hot mix asphalt (Roberts, et al., 1996). The rolling thin film oven test (RTFO) is a conditioning procedure that simulates the age hardening asphalt undergoes during the production and construction of HMA. The conditioning procedure is used to determine the effect of heat and air on a moving film of asphalt and to evaluate the resistance to aging during the production and construction of hot mix asphalt structures.

\section{Summary of Method}

The AASHTO T240 standard test method requires that the RTFO continuously expose asphalt specimens to both heat $\left(163{ }^{\circ} \mathrm{C}\right)$ and airflow $(4000 \mathrm{ml} / \mathrm{min})$ for 85 minutes to achieve the accelerated age hardening of the asphalt. Eight asphalt specimens (35 grams each) can be conditioned during each RTFO procedure. The RTFO conditions the asphalt specimen for further conditioning and physical testing. The PG specifications of AASHTO MP1 limit the mass loss due to RTFO conditioning to one percent or less.

\section{Equipment}

\section{Hardware}

The Despatch RTFO evaluated during this research is shown in Figure 2.2. A RTFO consists of an oven chamber that houses a vertical circular carriage. The carriage, which holds eight RTFO specimen bottles, rotates about its center. A single air jet is located in the oven. Hot air is blown into the center of each RTFO bottle as it passes in front of the jet. A fan continually circulates the air within the oven chamber. The system requires a clean, dry, and pressurized air supply.

The AASHTO T240 specifications require that the RTFO specimen bottles are $139.7 \mathrm{~mm}$ long and $64.0 \mathrm{~mm}$ in outside diameter. The opening at the end of each bottle is $31.8 \mathrm{~mm}$ in diameter. The bottles are made of heat resistant glass with a $2.4 \mathrm{~mm}$ wall thickness. 


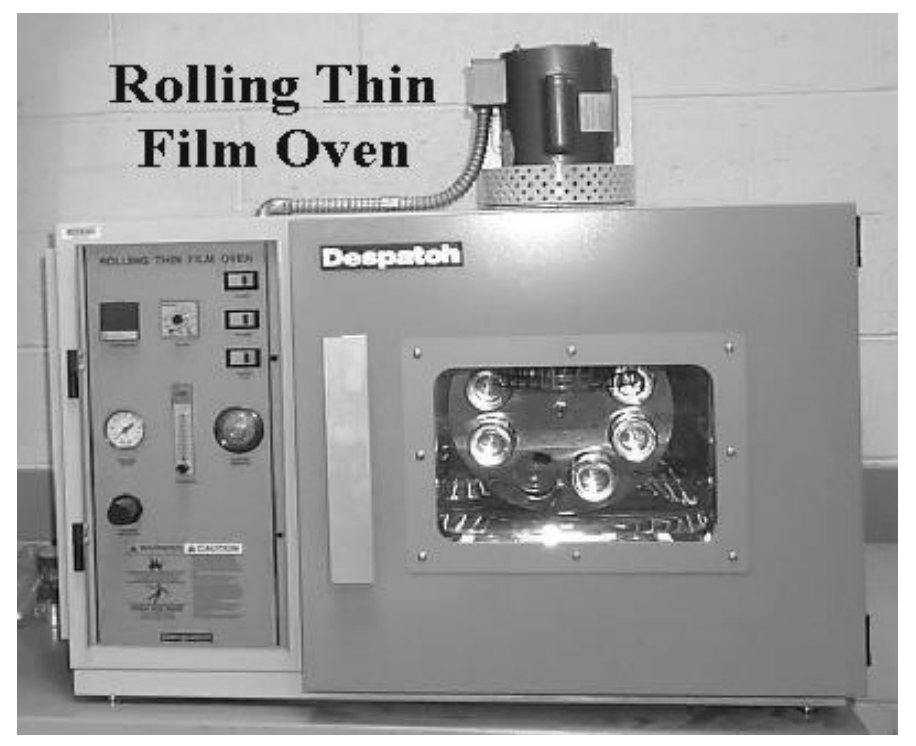

Figure 2.2 Despatch Rolling Thin Film Oven

\section{Data Collection}

No data is collected during the RTFO conditioning procedure. The average asphalt mass loss, however, is recorded at the conclusion of the procedure. The average asphalt mass loss is determined by weighing the asphalt samples from two RTFO bottles before and after the conditioning procedure and computing the difference as follows:

$$
\text { Percent Change in Mass }=\frac{(\text { Initial Mass }- \text { Final Mass })}{\text { Initial Mass }} \times 100 \%
$$

\section{Testing Protocol}

Test Specification

AASHTO T240-98 Effect of Heat and Air on Rolling Film of Asphalt defines the procedures followed during RTFO conditioning.

\section{Test Sample and Equipment Preparation Summary}

The RTFO oven is preheated to the $163{ }^{\circ} \mathrm{C}$ test temperature 16 hours prior to the test. The carriage rotation is set at $15 \mathrm{rpm}$ and the air jet flowrate is set at $4000 \mathrm{ml} / \mathrm{min}$. At least 350 grams of neat asphalt is heated until fluid to pour. Upon reaching the desired consistency, 35 grams of the heated asphalt is poured into each RTFO specimen bottle 
and then allowed to cool to room temperature. If change in mass determination is desired, two RTFO bottles first must be weighed empty. The bottles are then filled with a 35 grams asphalt sample and placed in a desiccator while cooling to room temperature. The filled RTFO bottles are weighed again upon cooling.

\section{Test Procedure Summary}

Once the 16-hour preheating requirement is complete and the asphalt samples have cooled to room temperature, the RTFO bottles are placed in the RTFO carriage and the test is initiated. The sample bottles remain in the oven for 85 minutes. The rotating carriage continuously exposes fresh asphalt to the heat and circulating air. The hot air is blown into the bottle with each pass in front of the air jet. The constant rolling action of the binder in the sample bottles prohibits the formation of an age inhibiting skin, unlike the previous thin film oven tests (Roberts, et al., 1996). The rolling action also benefits in the conditioning of modified asphalt since the modifiers remain dispersed in the binder.

At the conclusion of the 85-minute conditioning procedure, the two specimen bottles designated for mass loss determination are removed from the RTFO and immediately placed in a desiccator while they cool to room temperature. The six remaining specimen bottles are removed from the RTFO and their asphalt residue is collected in a container. The AASHTO procedure requires that the final RTFO bottle be removed from the RTFO within five minutes of the removal of the first bottle. Once the asphalt sample from the final bottle is collected in the container, the container is covered and stored for further conditioning and/or testing.

The two bottles designated for mass loss determination are weighed once they have cooled to room temperature and the average mass loss from the two bottles is calculated. The asphalt from these bottles is then discarded. 


\section{Unit Calibration}

The rolling thin film oven requires a periodic verification of the RTFO flow meter. The required $4000 \mathrm{ml} / \mathrm{min}$ airflow is verified by using wet-test meter or other displacement methods. The oven temperature controller must be periodically verified. The oven temperature RTFO is measured by a proportional control thermostat-sensing element. An ASTM Loss on Heat Thermometer 13C is located within the oven as well. The thermostat-sensing element is initially calibrated via a temperature offset to the ASTM thermometer. The temperature offset is programmed into the temperature controller. Agreement between the ASTM thermometer temperature and the temperature controller display should be verified prior to each RTFO conditioning process.

\subsection{Pressure Aging Vessel}

\section{Introduction}

\section{Purpose and Scope}

The pressure aging vessel (PAV) simulates the age hardening of asphalt during the first 5-10 years of pavement service life (Roberts, et al., 1996). The pressure aging vessel conditioning allows further testing by the dynamic shear rheometer and bending beam rheometer to evaluate the binder's performance following aging.

\section{Summary of Method}

While the Pressure Aging Vessel procedure is often referred to as a test, it is only a conditioning procedure. The AASHTO PP1 standard test method requires that the PAV expose RTFO-aged residue to high pressure, 2.1 MPa, and high temperature over a 20hour period to achieve accelerated hardening of the asphalt. The conditioning temperature, which is dependant upon the asphalt grade being conditioned, ranges from $90{ }^{\circ} \mathrm{C}$ to $110{ }^{\circ} \mathrm{C}$. Ten asphalt specimens, 50 grams each, can be conditioned with each PAV run. The residue from the PAV is degassed in a vacuum oven immediately following conditioning. 


\section{Equipment}

\section{Hardware}

The primary components of a pressure aging vessel include a pressure vessel and a heating unit. Two different types of pressure aging vessel units are specified in the AASHTO test method. One type of pressure aging vessel unit is an integral system in which the pressure vessel is permanently enclosed in an oven. The other available type is set up such that the pressure vessel is a separate unit placed in a forced draft oven. Both types of units are equipped with a pressure regulator and transducer, temperature controller, thermocouple or resistance thermal detector (RTD) and a data acquisition system. The ATS pressure aging vessel unit evaluated by this research is shown in Figure 2.3. The pressure vessel houses a sample rack that holds 10 asphalt specimen pans. The pressure aging vessel unit requires a separate compressed air cylinder to supply clean, dry air to the pressure vessel. A vacuum oven is also required for degassing the PAV-aged residue following the pressure aging vessel conditioning procedure.

\section{Data Collection}

The pressure aging vessel conditioning procedure requires no data collection. AASHTO PP1 does require the monitoring of the pressure and temperature, however, at regular intervals throughout the duration of the conditioning procedure. The aging temperature must be reached within 2 hours of the initiation of the procedure, or the asphalt samples must be discarded. The pressure must remain at $2.1 \pm 0.1 \mathrm{MPa}$ and the temperature within $\pm 0.5^{\circ} \mathrm{C}$ of the test temperature during the conditioning procedure. The pressure must be reduced to atmospheric pressure within $9 \pm 1$ minutes at the completion of the 20-hour conditioning procedure. The data acquisition system automatically monitors the pressure and temperature. The pressure and temperature data log can be reviewed on the ATS 504D pressure aging vessel; however, a hardcopy of the data cannot be printed. 


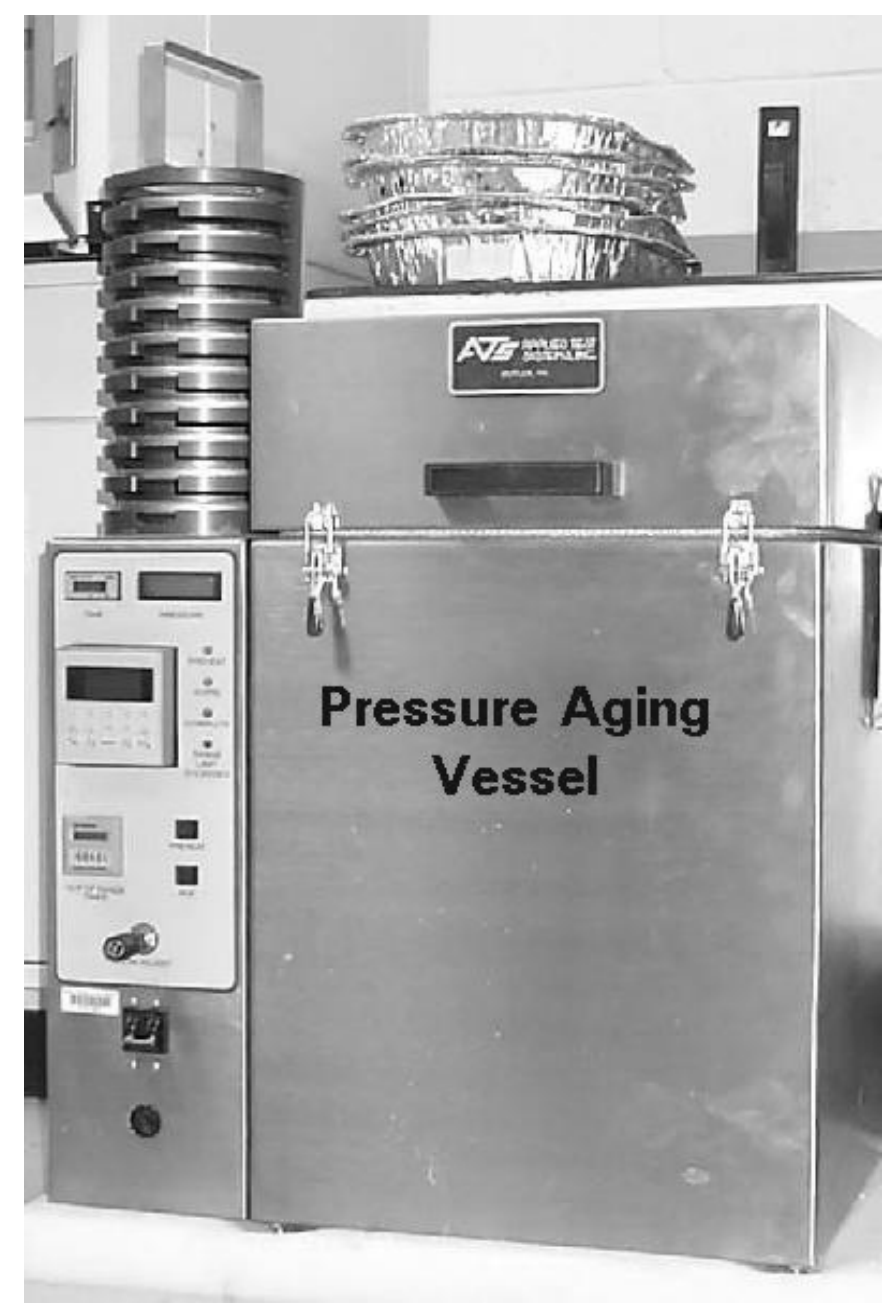

Figure 2.3 ATS Pressure Aging Vessel

\section{Testing Protocol}

Test Specification

AASHTO PP1-98 Standard Practice for Accelerated Aging of Asphalt Binder Using a Pressurized Aging Vessel (PAV) defines the protocol required for PAV conditioning of asphalt cement.

\section{Test Sample and Equipment Preparation Summary}

The pressure aging vessel unit is preheated to the required conditioning temperature. The test temperatures specified in AASHTO MP1 include $90{ }^{\circ} \mathrm{C}, 100{ }^{\circ} \mathrm{C}$, or $110{ }^{\circ} \mathrm{C}$, depending on the asphalt grade. Asphalt grades PG 46 and PG 52 are 
conditioned at $90{ }^{\circ} \mathrm{C}$. Asphalt grades PG 58, PG 64, PG 70, PG 76, and PG 82 are conditioned at $100{ }^{\circ} \mathrm{C}$. Asphalt grades PG 70, PG 76, and PG 82 are conditioned at $110{ }^{\circ} \mathrm{C}$ for desert climates (see Table 2.4). The pressure controller is preset to the 2.1 MPa test pressure. A covered container of RTFO-aged residue is heated in an oven until fluid to pour. A 50-g sample of the heated RTFO residue is poured into each specimen pan.

\section{Test Procedure Summary}

The PAV specimen pans are loaded in the sample rack and placed into the pressure vessel/oven unit. The conditioning test is initiated and the pressure vessel automatically pressurizes. The 20 -hour conditioning period begins once the temperature stabilizes following the vessel pressurization. The test temperature and pressure are maintained throughout the 20-hour conditioning process. The pressure vessel automatically depressurizes at the conclusion of the procedure.

The specimen pans are removed from the pressure vessel and placed in a preheated to $163{ }^{\circ} \mathrm{C}$ oven for 15 minutes. The specimen pans are removed from the oven and the PAV-aged residue is collected in a container. The container of PAV-aged asphalt is immediately placed in a vacuum oven, preheated to $170{ }^{\circ} \mathrm{C}$, without the vacuum applied. Following a 10-minute equilibration period, the vacuum valve is opened to reduce the oven pressure to $15 \mathrm{kPa}$ absolute. The vacuum is maintained for 30 minutes. At the end of 30 minutes, the vacuum is released and the container of PAV-aged asphalt is removed. The container is inspected for air bubbles on the surface of the asphalt residue. Any air bubbles present are removed with a hot knife or flashed with a torch. The container is then covered and the sample is stored for subsequent physical testing.

\section{Unit Calibration}

The pressure aging vessel must have the resistance thermal detector (RTD) within the pressure vessel and the pressure gauge calibrated every six months. These calibration procedures are typically performed by a commercial calibration service. 


\subsection{Rotational Viscometer}

\section{Introduction}

\section{Purpose and Scope}

Asphalt binders must remain sufficiently fluid, or workable, at the high temperatures necessary during the plant mixing, field placement and compaction of hot mix asphalt (Roberts, et al., 1996). The binder reaches temperatures exceeding $135^{\circ} \mathrm{C}$ during these procedures. The rotational viscometer (RV) is used to evaluate binder in an unconditioned or neat state. This represents the state of material in the tank at the asphalt plant. The rotational viscometer measures the rheological properties of asphalt binders to evaluate their pumpability during delivery and plant operations.

The rotational viscometer is also used to establish equiviscous temperature ranges for selecting HMA mixing and compaction temperatures (Asphalt Institute, 2001). Mixing and compaction temperatures are determined from viscosity-temperature graphs. A typical log-log viscosity vs. log temperature graph is shown in Figure 2.4 (Asphalt Institute, 2003). Viscosities at two temperatures are plotted on the log-log viscosity vs. $\log$ temperature graph. The equiviscous temperature range for mixing and compaction correspond to where the plotted line passes through the respective viscosity range.

\section{Summary of Method}

The rotational viscometer determines the asphalt viscosity by measuring the torque necessary to maintain a constant rotational speed of a cylindrical spindle submerged in an asphalt specimen held at a constant temperature, as per the AASHTO TP48 standard test method. Unlike the capillary viscometers used with the viscositygraded method, the rotational viscometer can evaluate modified asphalt binders (Anderson, et al., 1994). The viscosity of asphalt binders can be measured within the range of $0.01 \mathrm{~Pa} \cdot \mathrm{s}$ (0.1 poise) to $200 \mathrm{~Pa} \cdot \mathrm{s}$ (2000 poise) (Petersen, et al., 1994b). 


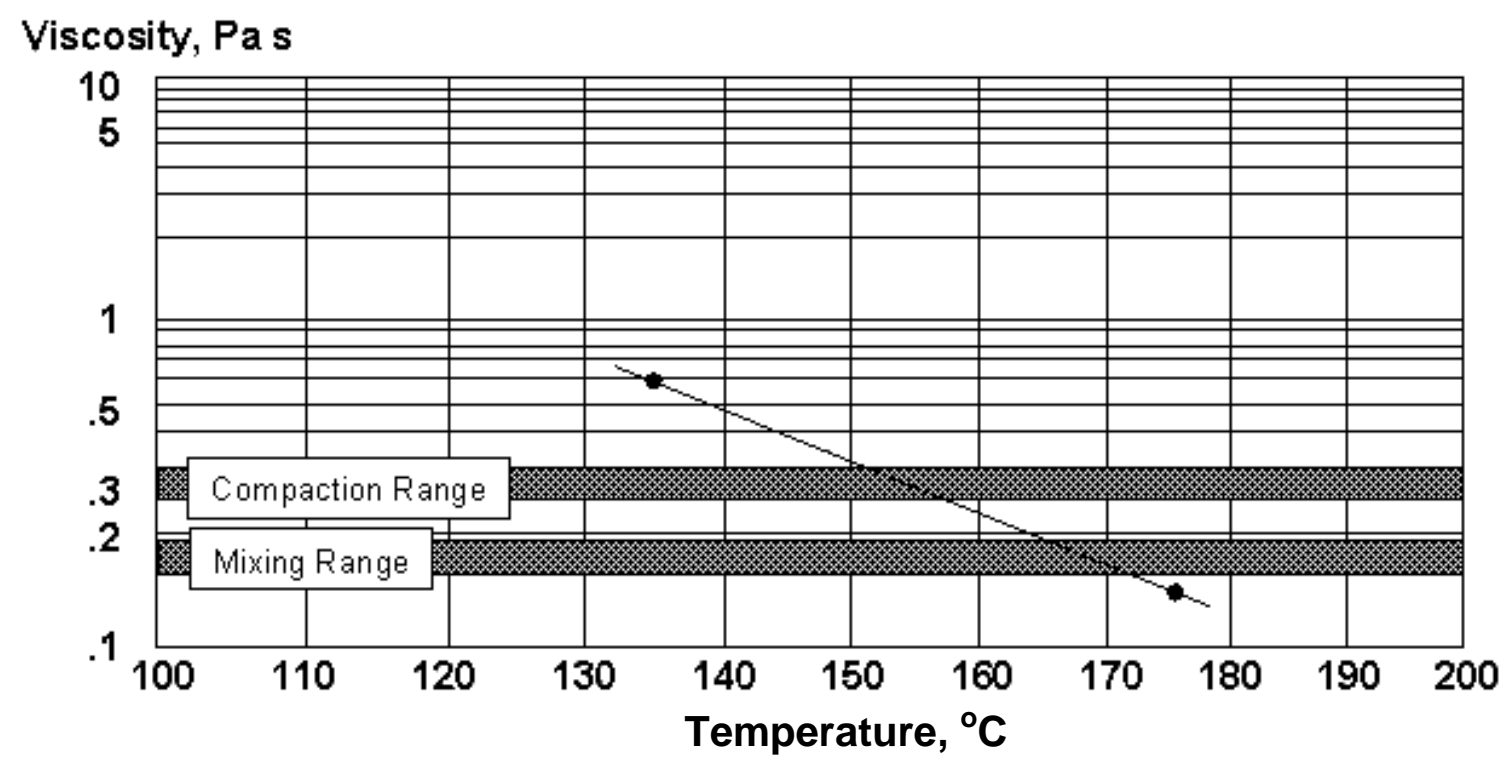

Figure 2.4 Typical Equiviscosity Curve for Asphalt Binder

\section{Equipment}

\section{Hardware}

The major components of a rotational viscometer system consist of a rotational viscometer, temperature controller, thermo-chamber and a personal computer (not required with some rotational viscometers). The rotational viscometer is a rotating spindle-type viscometer. It is comprised of a rheometer head and base unit. The rheometer head unit consists of a motor, rotational transducer, spindle, digital readout display and a control panel. The base unit acts as interface between the rheometer head and the personal computer. The thermo-chamber is a heating chamber that houses the asphalt sample holder during the test. The temperature within the thermo-chamber is monitored by a resistance thermal detector (RTD) and controlled by the temperature controller unit. The Brookfield DV-III rotational viscometer evaluated during this research is shown in Figure 2.5. 


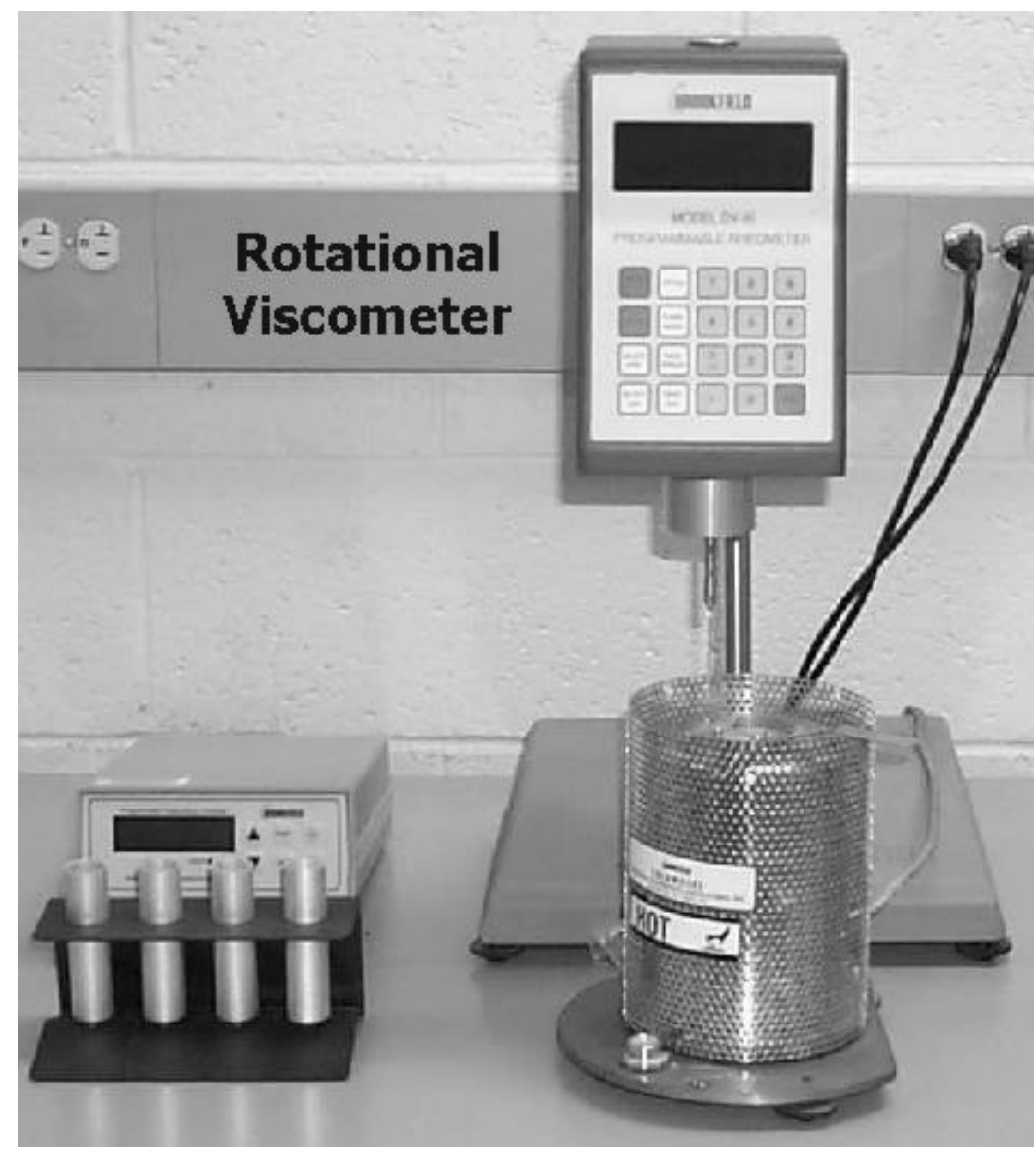

Figure 2.5 Brookfield DV-III Rotational Viscometer

\section{Data Collection}

A rotational transducer within the rheometer head measures the torque required to turn the spindle. The electronic circuitry within the base unit automatically converts the measured torque to viscosity and sends the test results to the computer. Viscosity readings are taken at one-minute intervals for three readings.

\section{Testing Protocol}

Test Specification

AASHTO TP48-97 Standard Test Method for Viscosity Determination of Asphalt Binder Using Rotational Viscometer defines the protocol for measuring the viscosity of asphalt binders at high temperatures. This test method does not cover procedures for measuring the temperature-viscosity relationship for asphalt binders. 


\section{Test Sample and Equipment Preparation Summary}

The test operating speed of the rotational viscometer is set. The temperature controller of the thermo-chamber is set at the test temperature and allowed to preheat. The sample holder and spindle are preheated in an oven heated to the test temperature as well. A test temperature of $135{ }^{\circ} \mathrm{C}$ is specified for evaluating pumpability in AASHTO MP1. Rotational viscosity tests at two temperatures are needed when creating viscositytemperature graphs for establishing equiviscous temperature ranges. The Asphalt

Institute recommends taking the first viscosity measurement at $135{ }^{\circ} \mathrm{C}$, and the second at $165{ }^{\circ} \mathrm{C}$ (Asphalt Institute, 2001). The lower temperature measurement is taken first as it is much quicker to raise the temperature in the thermo-chamber than to cool the sample for the second viscosity measurement. Thus, prior to conducting tests for equiviscous temperature ranges, the thermo-chamber should be preheated at the lower temperature. Likewise, the sample holder and spindle are preheated in an oven heated to the lower temperature.

A container of neat asphalt is placed in the oven and heated until fluid to pour. The heated asphalt is poured into the sample holder to a level that will just cover the upper conical portion of the spindle when it is lowered into the sample holder.

\section{Test Procedure Summary}

The sample holder is placed in the preheated thermo-chamber. The spindle is removed from the oven, attached to the viscometer, and lowered into the asphalt sample. The system is allowed to equilibrate for 30 minutes and the test is initiated.

Three viscosity measurements are taken at each test temperature. Thus, when evaluating asphalt pumpability, three viscosity measurements are taken at $135{ }^{\circ} \mathrm{C}$. When establishing equiviscous temperature ranges, three measurements are taken at the lower temperature and at the higher temperature.

If the displayed torque is out of the allowable viscosity range for the selected spindle and operating speed, the spindle or speed is changed, as per the manufacturer's 
recommendations, and the test is restarted with a new asphalt specimen. The test is terminated at the conclusion of the viscosity readings. The sample holder is removed from the thermo-chamber and the asphalt specimen is discarded.

\section{Unit Calibration}

The test method requires the verification of the RV temperature controller. An asphalt sample is placed in the testing chamber and heated to the test temperature. The indicated temperature of the sample is verified via an ASTM thermometer. In addition to the temperature controller verification, the accuracy of the rotational transducer is checked by performing a viscosity test on a standard viscosity reference fluid.

\section{Theory of Analysis}

Most asphalt binders behave like Newtonian fluids when heated to temperatures exceeding $100{ }^{\circ} \mathrm{C}$ (Bahia and Anderson, 1995a). A Newtonian fluid is characterized by a totally viscous response, in which the viscosity is independent of the rate of shear strain. Since asphalt has a totally viscous response at high temperatures, it is sufficient to characterize the workability of the asphalt during mixing and construction with a measure of its viscosity.

The working equations relating shear stress to torque and shear rate to angular velocity are as follows (Petersen, et al., 1994b and Whorlow, 1992):

$$
\begin{aligned}
& \text { Shear Rate }\left(\mathrm{s}^{-1}\right) \quad \dot{\gamma}=\omega\left[\frac{2 R_{c}^{2}}{\left(R_{c}^{2}-R_{s}^{2}\right)}\right] \\
& \text { Shear Stress (dynes } \left./ \mathrm{cm}^{2}\right) \quad \tau=\frac{M}{2 \pi R_{s}^{2} L}
\end{aligned}
$$

where: $\omega=$ angular velocity of spindle $(\mathrm{rad} / \mathrm{s})$

$\mathrm{R}_{\mathrm{c}}=$ radius of sample holder $(\mathrm{cm})$

$\mathrm{R}_{\mathrm{s}}=$ radius of spindle $(\mathrm{cm})$

$\mathrm{M}=$ applied torque (dyne-cm)

$\mathrm{L}=$ effective length of spindle $(\mathrm{cm})$ 
The viscosity is calculated by the following relationship:

$$
\text { Viscosity (poise) } \quad \mu=\frac{\tau}{\dot{\gamma}}
$$

The unit of viscosity may be converted to pascal-seconds $(\mathrm{Pa} \cdot \mathrm{s})$ using the conversion 1 centipoise $=0.001 \mathrm{~Pa} \cdot \mathrm{S}$.

\section{Parameter Specifications}

AASHTO MP1 requires a maximum viscosity limit of $3 \mathrm{~Pa} \cdot \mathrm{s}$ when tested at $135{ }^{\circ} \mathrm{C}$. The maximum limit ensures that the asphalt binder is sufficiently fluid for pumping during delivery and plant operations.

New mixing and compaction viscosity specifications were not developed during the SHRP research (Asphalt Institute, 2001). The industry standard for the past twenty years has been the viscosity ranges of $170 \pm 20$ centistokes for mixing temperatures and $280 \pm 30$ centistokes for compaction temperatures. The same viscosity ranges are still recommended for Superpave; however, the units have been converted the Pascal-second metric equivalent; $0.17 \pm 0.02 \mathrm{~Pa} \cdot \mathrm{s}$ for mixing and $0.28 \pm 0.02 \mathrm{~Pa} \cdot \mathrm{s}$ for compaction.

\subsection{Dynamic Shear Rheometer}

\section{Introduction}

\section{Purpose and Scope}

Roberts, et al. (1996) and Bahia and Anderson (1995a) present a description of the purpose and scope of the dynamic shear rheometer test, which served as the basis for the following section. Asphalt concrete pavements can be prone to wheel path rutting in the early stages of their life. Repeated traffic loads increase the consolidation of the pavement structure after construction, thus causing depressions in the traffic wheel paths. Traffic induced rutting can also result from the lateral flow of the asphalt concrete materials in the wheel paths. Although the rutting potential of asphalt concrete pavements is influenced primarily by the quality of construction (compaction), mix design, and aggregate angularity and texture, the consistency of the binder must still be 
considered. Rutting is more prevalent at high service temperatures due to the increased fluidity of the asphalt. The rutting potential of HMA pavements decreases with time since the asphalt binder stiffens with age hardening.

Asphalt concrete pavements also develop the potential for fatigue cracking later in their service life. Repeated load-associated stresses are the primary cause of fatigue cracking. Asphalt binder properties play a major role in the fatigue life of thin HMA pavements. Excessive asphalt binder hardening can significantly decrease the fatigue life of thin HMA pavements.

The dynamic shear rheometer (DSR) was adopted to characterize the viscoelastic behavior of asphalt binders at intermediate and high service temperatures. The DSR provides an indication of the rutting resistance of asphalt cement immediately following construction. Resistance to rutting at high service temperatures in the early stages of pavement life is also evaluated. The DSR also provides an indication of the resistance to fatigue cracking at immediate service temperatures in the later stages of service life.

\section{Summary of Method}

The viscoelastic properties of asphalt are determined by evaluating the behavior of an asphalt specimen when subjected to oscillatory (sinusoidal) stresses. The AASHTO TP5 standard test method requires that a thin asphalt specimen be sandwiched between two parallel metal plates held in a constant temperature medium. One plate remains fixed while the other oscillates, at an angular frequency of 10 radians per second for 10 cycles, with respect to the other. The dynamic shear rheometer evaluates the specimen's response to the sinusoidal stresses and calculates the asphalt's complex shear modulus and phase angle. The complex shear modulus and phase angle of a binder, which are indicators of an asphalt's resistance to shear deformation in the viscoelastic region, help predict the rutting potential and fatigue life of hot mix asphalt pavements (Bahia and Anderson, 1995a). 


\section{Equipment}

\section{Hardware}

A dynamic shear rheometer system includes the rheometer, temperature controller, data acquisition unit and a personal computer. The rheometer itself consists of a loading assembly, motor, load and strain transducer, parallel plates (an upper oscillatory spindle plate and a lower fixed plate) and an environmental control chamber with a resistance thermal detector (RTD) mounted inside. The Bohlin Instruments DSR II dynamic shear rheometer evaluated during this research is shown in Figure 2.6.

There are two types of dynamic shear rheometers: controlled stress and controlled strain. Controlled stress rheometers apply a sinusoidally varying stress and determine the resulting strain. Controlled strain rheometers apply a sinusoidally varying strain and determine the resulting stress.

The DSR uses two different sized parallel plate sets for evaluating asphalt binder: 8-mm diameter plates and 25-mm diameter plates. The $25-\mathrm{mm}$ plates are used with RTFO-aged asphalt specimens when evaluating the rutting resistance parameter. The 8mm plates are used with PAV-aged asphalt specimens when evaluating the fatigue cracking resistance parameter. Silicone molds are available for making asphalt test specimens for use with both the $25-\mathrm{mm}$ plates and the 8 -mm plates.

The environmental chamber houses the parallel plates, a platinum resistance thermometer, the test specimen and the medium used for heating or cooling the asphalt specimen to a constant test temperature. Two types of environmental chambers are available for controlling the asphalt specimen temperature. One type uses a circulating gas medium (nitrogen or dried air) to surround the test specimen. The other type of environmental chamber uses a water bath to surround the test specimen. A circulating bath unit is required if a fluid medium is used. 


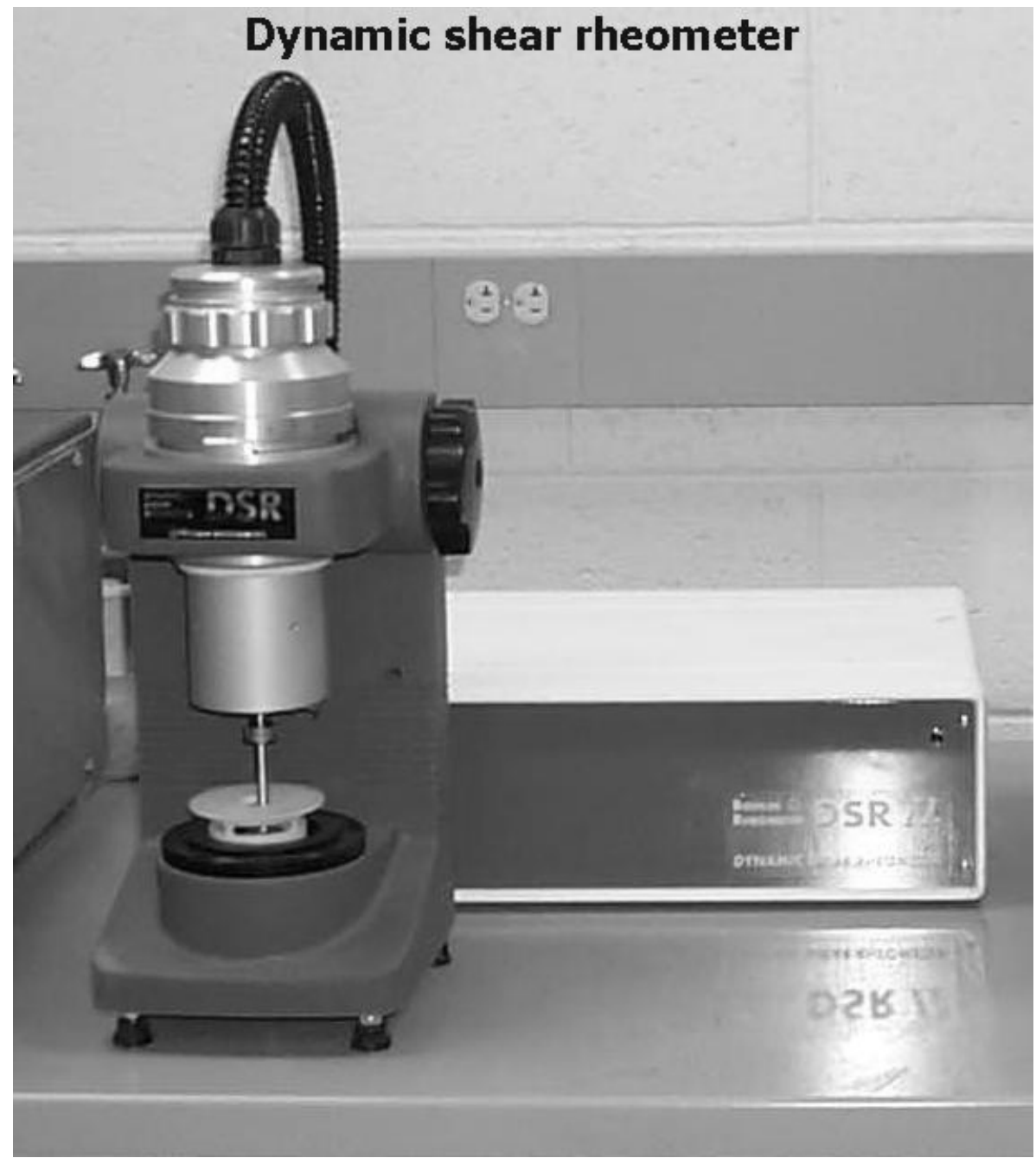

Figure 2.6 Bohlin Instruments DSR II Mechanical Dynamic Shear Rheometer

\section{Data Collection}

The data acquisition unit records the test temperature, applied torque, loading frequency and deflection angle during the 10 cycles of the test and sends the test data to the personal computer. The computer software reduces the data and calculates the shear stress, shear strain, complex modulus and phase angle. 


\section{Testing Protocol}

\section{Test Specification}

AASHTO TP5-98 Standard Test Method for Determining the Rheological Properties of Asphalt Binder Using a Dynamic Shear Rheometer (DSR) defines the protocol for conducting the dynamic shear rheometer test.

\section{Test Sample and Equipment Preparation}

The system software is initialized and the test parameters entered. The test temperature is set, as per AASHTO MP1 criteria. The test temperature selected depends on the asphalt type and the performance parameter. For rutting resistance evaluation, neat and RTFO-aged asphalt binders are tested at high service temperatures. Test temperatures range, depending on the grade of asphalt, from $46{ }^{\circ} \mathrm{C}$ to $82{ }^{\circ} \mathrm{C}$. PAV-aged asphalt is tested at intermediate service temperatures for fatigue life evaluation. Test temperatures range from $4{ }^{\circ} \mathrm{C}$ to $40{ }^{\circ} \mathrm{C}$.

The loading angular frequency is set to 10 radians per second. The shear stress amplitude or shear strain amplitude, for controlled stress mode testing or controlled strain mode testing, respectively, is set. The input target values for the shear stress amplitude and the shear strain amplitude depend on the type of asphalt being tested, as shown in Table 2.5.

\begin{tabular}{|lcc|}
\cline { 1 - 2 } Asphalt Tested & Target Shear Strain, \% & Target Shear Stress, kPa \\
\cline { 2 - 3 } Neat & 12 & 0.12 \\
RTFO-Aged & 10 & 0.22 \\
PAV-Aged & 1 & 50 \\
\hline
\end{tabular}

\section{Table 2.5 Target Shear Stress and Strain Values}

The appropriate plate size is selected, 25-mm diameter plates for neat and RTFOaged asphalt and 8-mm diameter plates for PAV-aged asphalt, and mounted on the rheometer. The gap between the upper and lower plates is then set. The gap between the 
plates is a critical test parameter. The accuracy of the gap measurement is directly related to the accuracy of the asphalt specimen evaluation. A micrometer wheel is used to measure the gap between plates (i.e. when the micrometer wheel is set on $1 \mathrm{~mm}$ and the upper plate is fully lowered, a 1-mm gap will be maintained between the plates). A gap measurement verification procedure, called setting the zero gap, must be conducted to ensure that the micrometer reading and the actual gap between the plates is the same. The zero gap is set by lowering the upper plate in small increments until the upper and lower plates just touch, or reach zero gap. The micrometer wheel is then set to zero when zero gap between the plates have been achieved. Before setting the zero gap, the temperature controller is turned on and the environmental chamber is preheated, or cooled, to the test temperature. The zero gap is set after the medium surrounding the plates stabilizes at the test temperature.

A covered container of the asphalt is placed in an oven and heated until fluid to pour. Two methods are accepted for fabricating an asphalt test specimen. One technique employs the use of the silicone mold. The heated asphalt is poured into the mold and allowed to cool. The asphalt specimen is then demolded and placed on the bottom loading plate. The other technique is to pour the heated asphalt directly onto one of the loading plates.

\section{Test Procedure Summary}

With the asphalt specimen properly placed on one of the loading plates, the upper plate is lowered to squeeze the asphalt specimen between the plates. The upper plate is lowered such that the gap between the plates is $0.05 \mathrm{~mm}$ greater than the test gap. The test gap for the $25-\mathrm{mm}$ plates and the $8-\mathrm{mm}$ plates is $1 \mathrm{~mm}$ and $2 \mathrm{~mm}$, respectively. The excess asphalt that is squeezed from between the plates is removed by trimming around the periphery of the plates. The upper plate is then lowered to the test gap. The asphalt specimen should slightly bulge around the periphery of the plates.

The trimmed specimen is brought to the test temperature, via the fluid or air medium, and allowed to equilibrate for 10 minutes. At the conclusion of the 10 -minute 
equilibrium period, the specimen is conditioned by applying the required stress or strain at the $10 \mathrm{rad} / \mathrm{sec}$ frequency for 10 cycles. The test is then run by applying the same stress or strain for an additional 10 cycles. The data from the second set of cycles are reduced and used to calculate the complex shear modulus and phase angle.

\section{Unit Calibration}

The calibration procedures for the dynamic shear rheometer include a resistance thermal detector (RTD) calibration/verification and overall verification calibration. The fluid bath RTD is initially calibrated by using a reference thermister that is inserted into a silicone wafer the size of an asphalt testing specimen. The thermister/wafer apparatus is inserted between the DSR testing plates in the fluid bath. The resistance created by the thermister is read by an ohms resistance meter and then converted to a temperature reading. An appropriate temperature correction to the DSR RTD temperature measurement is applied if the measurement is not within $\pm 0.1{ }^{\circ} \mathrm{C}$ of the reference thermister measurement. The RTD measurement must be periodically verified using the reference thermister.

The overall calibration of the DSR is checked by performing a test on a specimen made from a standard viscosity fluid. The complex modulus measurement and the test frequency are used to determine the viscosity of the reference fluid. The resulting viscosity value must be within an allowable range or recalibration of the DSR by the manufacturer is required.

\section{Theory of Analysis}

Since the dynamic shear rheometer evaluates asphalt binder properties at intermediate to high service temperatures, the binder responses are in the viscoelastic range (Bahia and Anderson, 1995a). Therefore, viscosity measurements alone are not sufficient to characterize the asphalt behavior. Properties need to be established which characterize the resistance to deformation and the relative distribution of the resistance between the elastic component and the viscous component. Dynamic, or oscillatory, testing is a common technique for evaluating viscoelastic behavior. 
The DSR evaluates the behavior of an asphalt specimen by subjecting it to oscillatory (sinusoidal) stresses. A thin asphalt specimen is sandwiched between two parallel metal plates held in a constant temperature medium. One plate remains fixed while the other oscillates, at an angular frequency $(\omega)$ of 10 radians per second for 10 cycles, with respect to the other. A complete DSR loading cycle is shown in Figure 2.7 (Roberts, et al., 1996). When torque from the DSR motor is applied, the oscillating plate moves from point A to point B. The plate then passes back through point A to point $\mathrm{C}$. The cycle of oscillation is completed as the plate passes back through point A again.

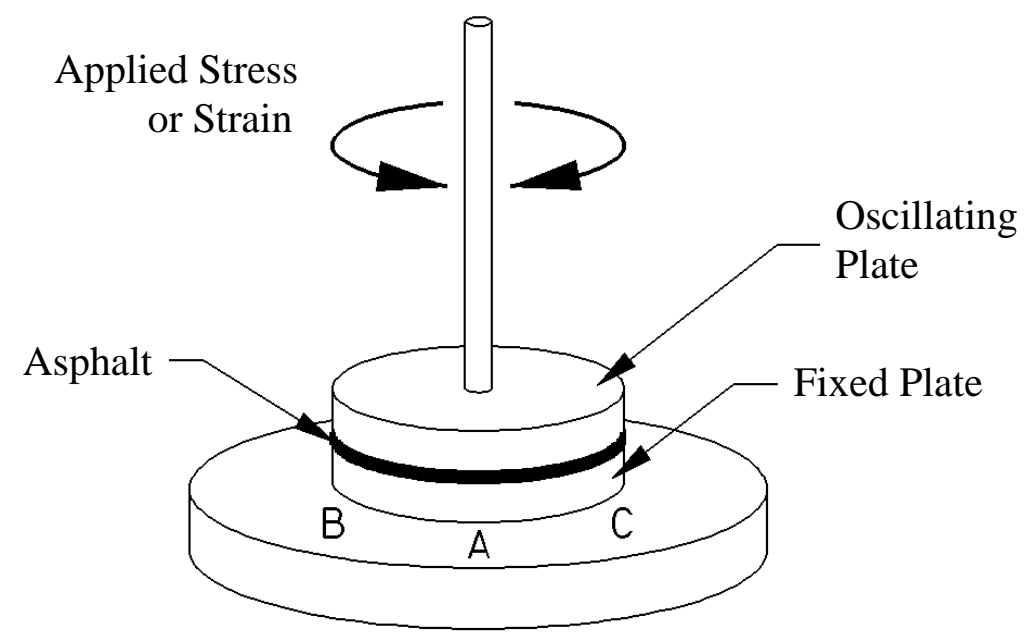

Figure 2.7 Configuration and Load Cycle of Dynamic Shear Rheometer

The $10 \mathrm{rad} / \mathrm{sec}$ angular frequency is equivalent to a frequency $(f)$ of 1.59 cycles per second $(1.59 \mathrm{~Hz})$, as per the relationship $\omega=2 \pi f$ (Petersen, et al., 1994a). The $10 \mathrm{rad} / \mathrm{sec}$ angular frequency corresponds, with sinusoidal loading, to a 0.1 second loading time, where loading time $(\mathrm{t})$ is determined from the relationship $\mathrm{t}=(2 \pi f)^{-1}$. The 0.1 -second loading time represents the loading time within a pavement structure resulting from the pass of a truck tire traveling at $50 \mathrm{mph}$. 
The basis for the 0.1-second loading time was not found in the literature.

However, the relationship between tire contact area and load duration presented in Huang (1993) may serve as the basis for this loading time. For example, the load per tire of a dual tire, 18-kip axle, is 4500 pounds. A tire pressure of 70 psi yields a tire contact area, Ac, of $64.29 \mathrm{in}^{2}$.

$$
\text { where, } \quad \text { Ac }=\frac{\text { Tire Load }}{\text { Tire Pressure }}
$$

For a combined contact area of the dual tires, the combined contact radius, Rc, of dual tires is $0.53 \mathrm{ft}$.

$$
\text { where, } \quad \mathrm{Rc}=\sqrt{2\left(\frac{\mathrm{Ac}}{\mathrm{p}}\right)}
$$

For a speed, $\mathrm{S}$, of $50 \mathrm{mph}$, the load duration, $\mathrm{D}$, is 0.087 seconds.

$$
\text { where, } \quad \mathrm{D}=\frac{12 \mathrm{Rc}}{\mathrm{S}}
$$

By comparison, tire pressures of 75 psi and 110 psi yield load durations of 0.084 seconds and 0.070 seconds, respectively. These times represent the duration of the tire patch contact. The actual load duration is greater than these values, however, due to the "stress wave" surrounding the tire. Thus, the above relationships yield loading times that are reasonably close to the 0.1 -second loading times documented in the literature.

Dynamic testing provides an indication of a binder's resistance to deformation and the elastic/viscous component distribution by determining the binder's complex modulus and phase angle (Roberts, et al., 1996). The complex modulus, $\mathrm{G}^{*}$, commonly referred to as $\mathrm{G}$ star, represents the total deformation resistance when loaded or sheared. 
The complex modulus is defined as the ratio of the absolute value of the peak-topeak shear stress to the absolute value of the peak-to-peak shear strain:

$$
\text { Complex Modulus (Pa) } \quad \mathrm{G}^{*}=\frac{\left|\tau_{\text {Max }}-\tau_{\text {Min }}\right|}{\left|\gamma_{\text {Max }}-\gamma_{\text {Min }}\right|}
$$

The following relationships are used calculate $\tau_{\max }$ and $\gamma_{\max }$ :

$$
\begin{aligned}
\tau_{\max } & =\frac{2 \mathrm{~T}_{\max }}{\pi \mathrm{r}^{3}} \\
\gamma_{\max } & =\frac{\theta_{\max } \mathrm{r}}{\mathrm{h}}
\end{aligned}
$$

where: $\tau_{\max }=$ absolute value of the peak-to-peak shear stress $(\mathrm{Pa})$

$$
\begin{aligned}
& \gamma_{\max }=\text { absolute value of the peak-to-peak shear strain }(\%) \\
& \mathrm{T}_{\max }=\text { maximum applied torque }(\mathrm{N}-\mathrm{m}) \\
& \mathrm{r}=\text { radius of specimen plate }(\mathrm{mm}) \\
& \theta_{\max }=\text { maximum deflection angle }(\mathrm{rad}) \\
& \mathrm{h}=\text { specimen height }(\mathrm{mm})
\end{aligned}
$$

The phase angle, $\delta$, represents the relative distribution between the elastic response and the viscous response to loading (Roberts, et al., 1996). The phase angle indicates the delayed strain response, or lag, of the binder to the applied shear stress, during steady state conditions.

A graphical description of the phase angle with respect to time, applied shear stress, and strain is shown in Figure 2.8. For the elastic response, Figure 2.8 (a), the shear strain sinusoidal curve mirrors the applied shear stress sinusoidal curve at all points from (O) to (B) (Roberts, et al., 1996). For the viscous response, Figure 2.8 (b), there is a gradual strain response to the applied shear strain from point $(\mathrm{O})$ to point $(\mathrm{A})$. The strain response has not yet reached steady state conditions. However, at all points from (B) to (D) the strain response has a consistent time lag of equal duration between the 
applied shear stress and the strain response. Thus, the strain response has reached steady state conditions and is "out-of-phase" with the applied shear stress.

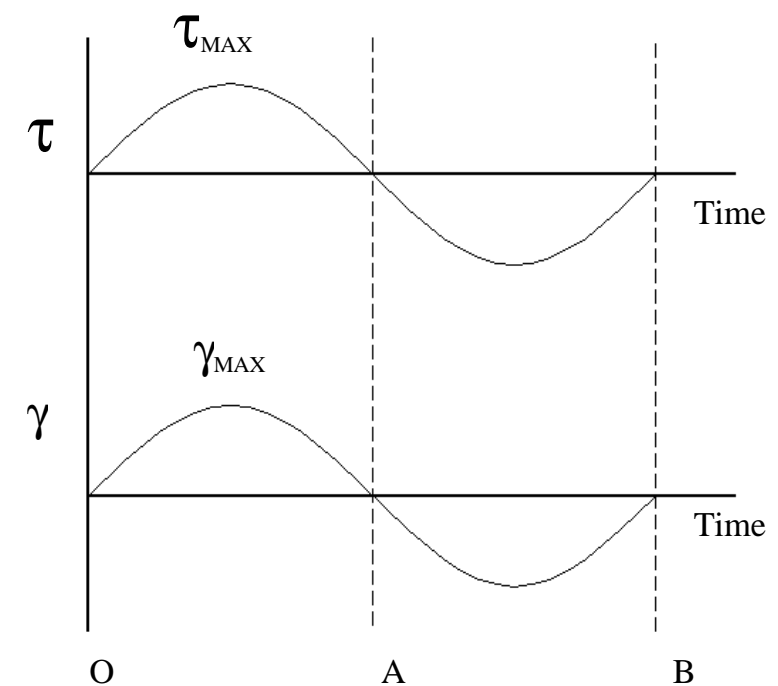

(a) Elastic Response $\delta=0^{\circ}$

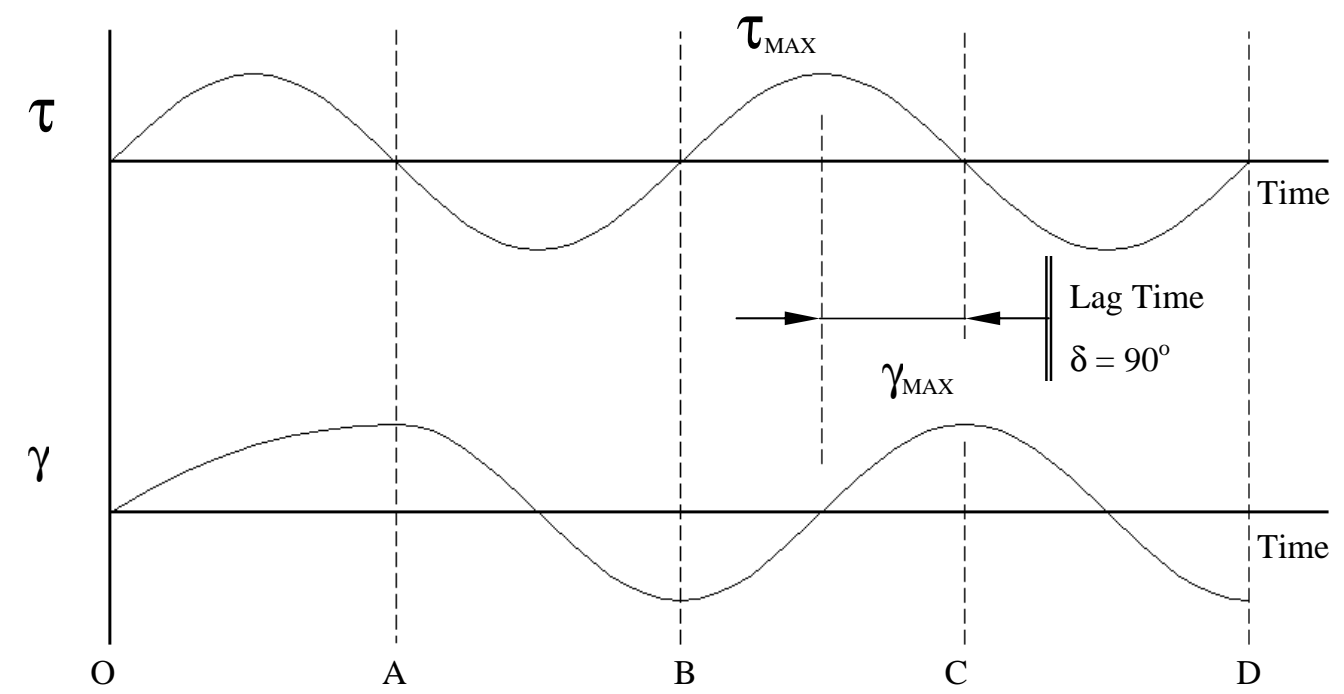

Steady State Response

(b) Viscous Response $\delta=90^{\circ}$

Figure 2.8 Phase Angles for Elastic and Viscous Materials 
The time lag at steady state conditions can be represented graphically as a $90^{\circ}$ shift between the maximum applied shear stress and the maximum shear strain. Thus, the strain response of a viscous material is $90^{\circ}$ "out-of-phase" with the applied shear stress, Figure 2.8 (b); therefore, the phase angle is 90 degrees. Conversely, there is no time lag between the applied shear stress and resultant strain response of an elastic material, Figure 2.8 (a); therefore, the strain response is "in-phase" with the applied shear stress and the phase angle is zero degrees.

The phase angle is the product of the time lag and the frequency, as shown in Equation 2-11.

Phase Angle, $\delta=(\Delta \mathrm{t} \times \omega)$

where: $\Delta \mathrm{t}=$ time lag $(\mathrm{sec})$

$\omega=$ angular frequency $(\mathrm{rad} / \mathrm{sec})$

The time lag between the maximum applied shear stress and the maximum shear strain response can be determined from Equation 2-11. For example, the phase angle for a totally viscous response at the test angular frequency of $10 \mathrm{rad} / \mathrm{sec}$ is 90 degrees. Substituting the phase angle and angular frequency into Equation 2-11 and solving for time lag:

$$
90^{\circ}=\frac{\pi}{2}=(\Delta \mathrm{t})(10 \mathrm{rad} / \mathrm{sec}) \quad \Delta \mathrm{t}=0.157 \mathrm{sec} .
$$

Thus, a 0.157 second lag exists between the maximum applied shear stress and the maximum shear strain response of a material having a totally viscous response. The time lag can be verified using the relationship between frequency $(f)$ and period, or cycle time (T), T= $f^{-1}$. An angular frequency of $10 \mathrm{rad} / \mathrm{sec}$ is equivalent to a frequency $(f)$ of 1.59 cycles per second $(1.59 \mathrm{~Hz})$. A $1.59 \mathrm{~Hz}$ angular frequency yields a period, or cycle time $(\mathrm{T})$, of 0.629 seconds as per the relationship $\mathrm{T}=f^{-1}$. One fourth of the 0.629 -second 
cycle time, which corresponds to $\frac{\pi}{2}$, is 0.157 seconds, which is consistent with Equation 2-11 and Equation 2.12.

A graphical description of the phase angle with respect to the complex modulus is shown in Figure 2.9 (Roberts, et al., 1996). The complex modulus consists of the storage modulus, G' and the loss modulus, G'. The storage modulus, which is the elastic (recoverable) component, represents the amount of energy stored in the sample during each loading cycle. The loss modulus, which is the viscous (non-recoverable) component, represents the amount of energy lost during each loading cycle. When the phase angle is zero degrees, elastic behavior, the complex modulus consists solely of the storage modulus. Likewise, when the phase angle is 90 degrees, viscous behavior, the complex modulus consists solely of the loss modulus. It is therefore necessary to determine both the complex modulus and the phase angle within the viscoelastic range of response to adequately characterize asphalt binders.
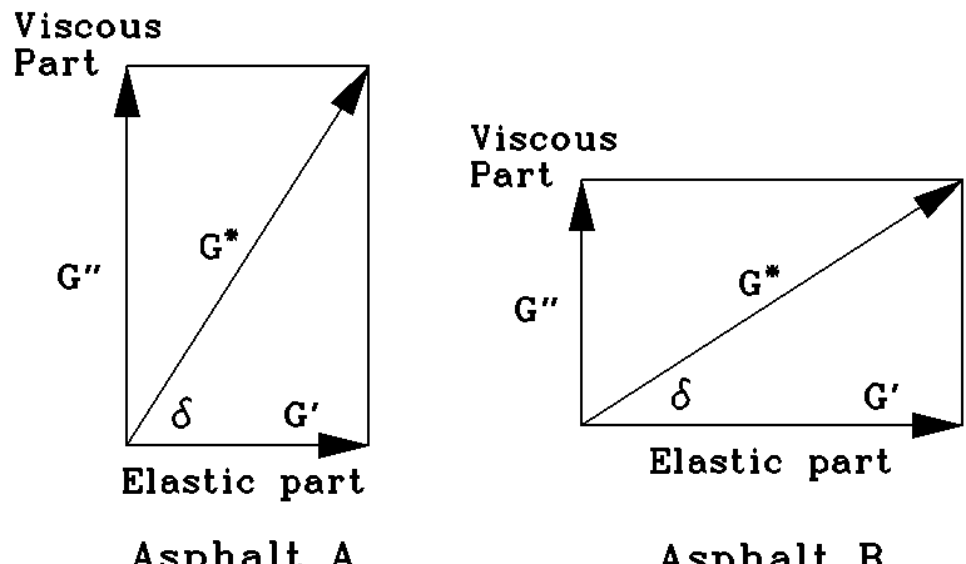

Asphalt A

\section{Asphalt B}

Figure 2.9 Relationship Between Complex Modulus and Phase Angle

The complex modulus must be measured within the linear viscoelastic range, which is the region of behavior in which the shear modulus is independent of shear stress or strain, to ensure test repeatability (Petersen, et al., 1994b). The limit of linear viscoelastic behavior is the point beyond which the complex modulus decreases to $95 \%$ 
of the measured value at zero-strain, as shown in Figure 2.10 (Petersen, et al., 1994b). The relationship derived during the SHRP research between the complex modulus and shear stress and strain within the linear viscoelastic range is shown in Equation 2-13 and Equation 2-14:

$$
\begin{array}{ll}
\text { Shear Stress }(\mathrm{kPa}) & \tau=0.12\left(\mathrm{G}^{*}\right)^{0.71} \\
\text { Shear Strain }(\%) & \gamma=12.0 /\left(\mathrm{G}^{*}\right)^{0.29}
\end{array}
$$

The target shear stress and shear strain parameters from Table 2.5 were established from Equation 2-13 and Equation 2-14, respectively. The AASHTO TP5 test method requires that the shear stress and shear strain be controlled to \pm 20 percent of the target values to ensure test repeatability.

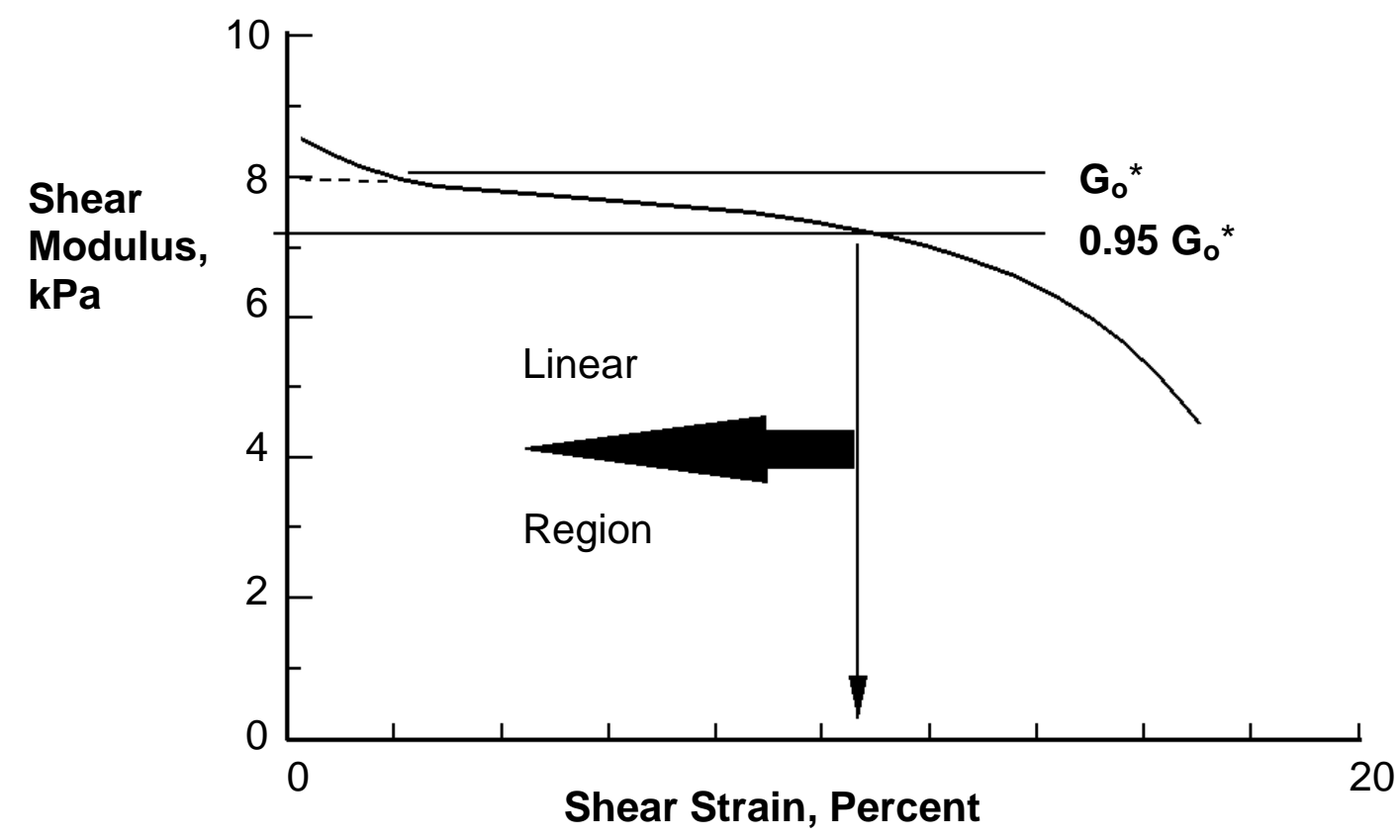

Figure 2.10 Graphical Description of Linear Viscoelastic Region

\section{Rutting Parameter}

The work of Bahia and Anderson (1995a) and Roberts, et al. (1996) served as the source of the following discussion on the rutting parameter development. The magnitude 
of the complex modulus and the degree of phase angle are required to determine the relationship between asphalt stiffness and the type of deformation: recoverable and nonrecoverable. This is especially true when considering rutting resistance at high service temperatures. A higher $\mathrm{G}^{*}$ and a lower $\delta$ are desired for rutting resistance. An asphalt with a high $\mathrm{G}^{*}$ is stiffer and provides increased resistance to deformation. An asphalt exhibiting a lower $\delta$ has a greater elastic component, thus allowing more of the total deformation to be recovered.

Rutting is assumed to be the primary result of deformations within the surface layer. Rutting is considered a stress-controlled, cyclic loading phenomenon. Work is being done to deform the surface layer with each loading cycle. A portion of the work is recovered in the elastic rebound of the surface layer, while the remaining work is dissipated through permanent deformation and heat. For a viscoelastic material, the work dissipated per loading cycle, $\mathrm{W}_{\mathrm{c}}$, is a function of stress and strain:

$$
\mathrm{W}_{\mathrm{c}}=(\pi)(\sigma)(\varepsilon)(\sin \delta)
$$

Since rutting is assumed to be a stress-controlled $\left(\sigma_{\mathrm{o}}\right)$, cyclic phenomenon, the work dissipated per loading cycle can be written as:

$$
\mathrm{W}_{\mathrm{c}}=(\pi)\left(\sigma_{\mathrm{o}}\right)(\varepsilon)(\sin \delta)
$$

By convention in the literature, the strain component in Equation 2-16 is computed as:

$$
\varepsilon=\frac{\sigma_{o}}{G^{*}}
$$

Note that Equation 2-17 does not follow conventional engineering notations as $\mathrm{G}^{*}$ is a shear modulus while e and s are traditionally used to indicate normal strains and normal stresses, respectively. 
Substituting Equation 2-17 into Equation 2-16, $\mathrm{W}_{\mathrm{c}}$ takes the final form:

$$
\mathrm{W}_{\mathrm{c}}=(\pi)\left(\sigma_{\mathrm{o}}^{2}\right)\left[\frac{1}{\mathrm{G}^{*} / \sin \delta}\right]
$$

The relationship $\mathrm{G}^{*} / \sin \delta$ was chosen as the parameter for SHRP specifications with respect to rutting. It can be seen in the preceding equation that an increase in $\mathrm{G}^{*}$ and decrease in $\sin \delta$ will both decrease the amount of work dissipated per loading cycle within a pavement's surface layer. This relationship follows the rationale that a binder with a high $\mathrm{G}^{*}$ value is stiffer, which increases its resistance to deformation, and a binder with a low $\sin \delta$ value is more elastic, whereby its ability to recover part of the deformation is increased.

\section{Fatigue Cracking Parameter}

Fatigue cracking is the primary pavement distress at intermediate service temperatures (Roberts, et al., 1996). Pavement fatigue cracking is considered a straincontrolled distress in thin pavement layers, less than 2 inches, because deformations in the asphalt layers are typically the result of poor subsurface layer support and not so much the effect of decreases in pavement stiffness (Huang, 1993). Pavement fatigue cracking is considered a stress-controlled distress in thick pavement layers, greater than 6 inches, as the pavement is the main load-carrying constituent. A combination of both stress-controlled and strain-controlled distresses exists with intermediate thickness HMA pavements.

Fatigue cracking occurs primarily in thin pavement layers; therefore, the distress is modeled as a strain-controlled phenomenon (Bahia and Anderson, 1995a and Roberts, et al., 1996). The work per cycle equation can be written for strain-controlled cyclic 
loading as follows:

$$
\mathrm{W}_{\mathrm{c}}=(\pi)(\sigma)\left(\varepsilon_{\mathrm{o}}\right)(\sin \delta)
$$

where $\varepsilon_{\mathrm{o}}$ is the applied strain amplitude. By convention in the literature, the stress component in Equation 2-19 is computed as:

$$
\sigma=\left(\varepsilon_{\mathrm{o}}\right)\left(\mathrm{G}^{*}\right)
$$

As with Equation 2-17, the relationship of Equation 2-20 does not follow conventional engineering notation. Substituting the above relationship into Equation 2-19 yields:

$$
\mathrm{W}_{\mathrm{c}}=(\pi)\left(\varepsilon_{\mathrm{o}}^{2}\right)\left[\mathrm{G}^{*} \sin \delta\right]
$$

The work done during a loading cycle can be dissipated by cracking, crack propagation, heat, and plastic flow. All of these dissipation mechanisms are damaging to pavement structures; therefore, it is necessary to limit the amount of energy dissipated. It can be seen in Equation 2-21 that strain-controlled work per cycle is directly proportional to $\mathrm{G}^{*} \sin \delta$. The $\mathrm{G}^{*} \sin \delta$ relationship was therefore selected as a parameter for the SHRP specification. By limiting the $\mathrm{G}^{*} \sin \delta$ parameter, decreasing $\mathrm{G}^{*}$ and/or $\sin \delta$, the energy dissipated per cycle is limited as well. This limiting parameter follows the rationale that a binder with a low $\mathrm{G}^{*}$ is softer, which allows it to deform without developing high stresses, and a binder with a low $\sin \delta$ will be more elastic, which enables the pavement structure to return to its original condition without dissipating energy.

\section{Parameter Specifications}

The parameter specification review by Roberts, et al. (1996) served as the basis for the following discussion. The Federal Highway Administration Asphalt Binder Expert Task Group (ETG) established the SHRP binder specifications for the DSR test parameters. The ETG is comprised of professionals from the asphalt industry, academia, 
and specification agencies. The ETG established three separate specification requirements for the DSR tests: minimum limits of $\mathrm{G} * \sin \delta$ for both unaged and RTFOaged binder tests, and a maximum limit of $\mathrm{G}^{*} \sin \delta$ for PAV-aged binder tests.

The minimum limit of $\mathrm{G}^{*} / \mathrm{sin} \delta$ for unaged asphalt testing is $1.0 \mathrm{kPa}$. The ETG selected $1.0 \mathrm{kPa}$ as the minimum limit based upon the results of performing DSR tests on AC-10 viscosity graded asphalts. Unaged AC-10 asphalts yielded $\mathrm{G}^{*} / \sin \delta$ values of approximately $1.0 \mathrm{kPa}$ when tested at the required 10 radians per second. Since AC-10 viscosity graded asphalts were providing reasonable service in moderate climates, the ETG concluded that the $1.0 \mathrm{kPa}$ value of $\mathrm{G}^{*} / \mathrm{sin} \delta$ for unaged asphalts was a reasonable specification limit.

The minimum limit of $\mathrm{G}^{*} / \mathrm{sin} \delta$ for RTFO residue is $2.2 \mathrm{kPa}$. This limit was derived from the $1.0 \mathrm{kPa}$ minimum limit for unaged binders. Following the review of RTFO test data, the average binder aging index was found to range from 2 to 2.5 , where the average binder aging index is defined as the binder viscosity after RTFO conditioning divided by the binder viscosity prior to RTFO conditioning. Thus, the binders became 2 to 2.5 times stiffer following RTFO conditioning, with 2.2 being the average of the range. The ETG, therefore, used the RTFO test data to establish the minimum $2.2 \mathrm{kPa}$ limit for RTFO-aged binders.

A maximum limit of $5000 \mathrm{kPa}$ was established for the $\mathrm{G}^{*} \sin \delta$ parameter. The ETG originally called for a maximum $3000 \mathrm{kPa}$; however, when this limit was investigated by testing 42 asphalt binders, only half would meet the initial specification. The ETG considered the initial specification too restrictive and chose to investigate a higher limit. After a review of the test data from 42 binders analyzed, it was noted that approximately $15 \%$ failed to achieve a $5000 \mathrm{kPa}$ value. The ETG considered the 5000 $\mathrm{kPa}$ value reasonable limit and established it as the maximum limit until field validation could be compiled and analyzed. 


\subsection{Bending Beam Rheometer \\ Introduction}

\section{Purpose and Scope}

The following section is based on the work of Bahia and Anderson (1995a) and Roberts, et al. (1996). Asphalt concrete pavements are susceptible to non-load associated distresses. Low-temperature cracking, commonly termed thermal cracking, is the most recognized non-load associated distress. Thermal cracking is caused by thermal shrinkage induced stresses resulting from environmental cooling. The asphalt binder in HMA pavements stiffens and shrinks during environmental cooling. The HMA layer, however, is restrained by the friction created with the underlying layers, which experience less shrinkage either because they remain warmer or because they have a smaller coefficient of thermal contraction. The friction-induced restraint creates tensile stresses within the asphalt layer that, if not relaxed by the flow of the asphalt binder, have the potential to exceed the tensile strength of the HMA layer and thus result in thermal cracking. The magnitude of stresses the HMA pavement layer experiences is dependent upon the stiffness of the asphalt binder, or its resistance to deformation, and its ability to relax those stresses by dissipating energy via permanent flow.

Thermal cracking can result from two different environmental conditions. The thermal distress can result from a single thermal cycle alone, in which a critically low temperature is encountered. This critical temperature is often referred to as the limiting stiffness temperature. Or, thermal cracking can result from thermal cycling where the temperature cycles up and down, but always remains above the limiting stiffness temperature.

The asphalt binder plays a critical role in the thermal cracking potential of HMA pavement. Hot mix asphalt pavements that have a high stiffness modulus at low temperatures are susceptible to thermal cracking. Asphalt binders become harder, or stiffer, as the ambient temperature decreases. Given that the stiffness of a HMA pavement is directly proportional to the asphalt binder stiffness, high binder stiffness at low temperatures increase the potential of thermal cracking. The bending rheometer was 
developed to determine the stiffness of asphalt binders at low service temperatures and to evaluate the binder's potential for thermal cracking.

\section{Summary of Method}

A PAV-aged asphalt beam specimen is subjected to a $980 \mathrm{mN}$ load for 240 seconds, as per the AASHTO TP1 standard test method. The beam is simply supported with the load applied at mid-span. The asphalt beam dimensions are $6.35 \mathrm{~mm}$ thick by $12.70 \mathrm{~mm}$ wide by $127 \mathrm{~mm}$ long. The asphalt beam is held at a constant low temperature throughout the test procedure. The load and deflection of the asphalt beam are measured and used to calculate the creep stiffness and the creep rate (m-value). The creep stiffness is an indicator of the specimen's ability to resist the constant creep load and the creep rate is the rate at which the creep stiffness changes with loading time (Roberts, et al., 1996).

\section{Equipment}

\section{Hardware}

The primary parts of the bending beam rheometer system consist of a loading frame, base unit, mechanical refrigeration unit, specimen molds and a personal computer. The specimen supports that the sample beam rests upon are mounted to the loading frame. A loading shaft assembly for applies the creep load. An air bearing and pneumatic piston controls the loading shaft's vertical movement. A linear variable differential transformer (LVDT) is attached to the loading shaft to measure the sample beam's deflection. A load cell is mounted with the loading shaft to measure the applied creep load. The ATS Bending Beam Rheometer evaluated during this research is shown in Figure 2.11.

The base unit houses a cooling fluid bath, system control and data acquisition electronics and air regulators that control the loading shaft. The cooling fluid bath houses the lower half of the loading frame, which includes the sample beam. The bath maintains the sample beam at a constant specified temperature during the test. The fluid bath must remain fluid and optically clear throughout the test temperature ranges. The mass density of the fluid bath is not to exceed $1.05 \mathrm{~kg} / \mathrm{m}^{3}$ at test temperatures, as per AASHTO TP1 
specifications, so that the sample beam remains neutrally buoyant during the test. A fluid mixture of 60 percent glycol, 15 percent methanol, and 25 percent water is recommended in AASHTO TP1 to achieve the required mass density. A bath agitator maintains the homogeneity of the fluid mixture and the homogeneity of the fluid temperature. The mechanical refrigeration unit cools the fluid bath. A resistance thermal detector is located in the fluid bath to monitor the temperature.

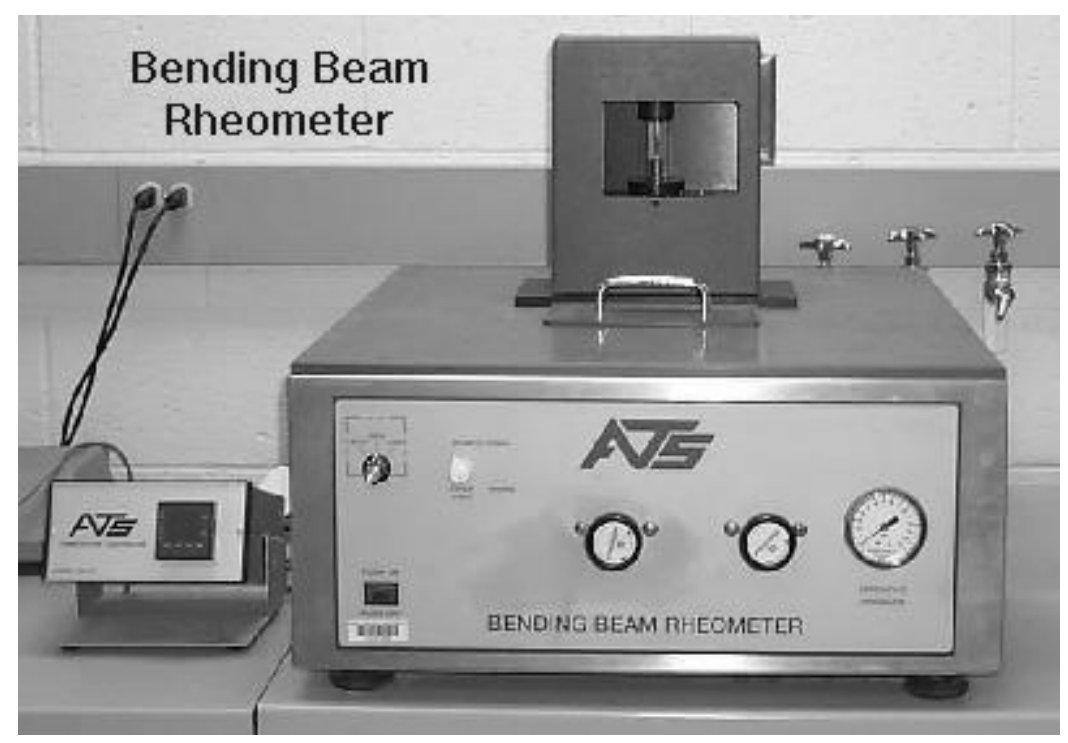

Figure 2.11 ATS Bending Beam Rheometer

The specimen molds form a $6.35 \mathrm{~mm}$ thick by $12.70 \mathrm{~mm}$ wide by $127 \mathrm{~mm}$ long asphalt beam. Aluminum specimen molds and silicone specimen molds are available for preparing the asphalt specimen beams. The specimen beam's geometry closely follows the guidelines for determining the flexural properties of elastomers established in ASTM D790.

\section{Data Collection}

The beam deflection, applied load and loading time is acquired by the data acquisition unit and sent to the personal computer during the test. Immediately following the test, the computer software automatically reduces the test data and computes the creep 
stiffness and creep rate. The creep stiffness and creep rates are reported at six loading times: $8,15,30,60,120$, and 240 seconds.

\section{Testing Protocol}

\section{Test Specification}

AASHTO TP1-98 Standard Test Method for Determining the Flexural Creep Stiffness of Asphalt Binder Using the Bending Beam Rheometer (BBR) defines the protocol used for conducting the bending beam rheometer test.

\section{Test Sample and Equipment Preparation}

The loading frame is placed in the fluid bath. The temperature controller is set to the test temperature and the fluid bath is subsequently cooled. The fluid bath is allowed to stabilize at the test temperature for at least 30 minutes prior to testing. A covered container of PAV-aged asphalt binder is placed in an oven and heated until fluid to pour. The heated binder is poured into the specimen mold and allowed to cool to room temperature.

Once the sample has cooled, the excess binder is trimmed from the top face of the mold with a hot spatula, such that the asphalt sample is flush with the top of the mold. The mold is placed in a freezer or ice bath for approximately 60 seconds so the sample beam can be easily demolded. Once the sample beam is demolded, it is immediately placed in the BBR fluid bath, which has been stabilized at the test temperature. The sample beam remains in the fluid bath for 60 minutes.

\section{Test Procedure Summary}

At the conclusion of the 60-minute conditioning period, the sample beam is placed on the loading frame specimen supports and the test is initiated. The BBR electronics and air regulators automatically control the load application and test duration. The sample beam is discarded at the conclusion of the 240-second test. 


\section{Unit Calibration}

The bending beam rheometer has many devices that need to be verified and calibrated. The load cell, linear variable differential transformer (LVDT), and resistance thermal detector (RTD) all require an initial calibration. The load cell is calibrated using a set of calibrated weights. The linear variable differential transformer (LVDT) is calibrated with a calibrated step-block. The BBR manufacturer provides both the calibrated weights and step-block. The fluid bath RTD must be initially verified via an ASTM thermometer. If the difference in temperature between the BBR display and the thermometer exceeds a specified value, the RTD requires recalibration. The recalibration is accomplished by establishing a temperature correction offset within the BBR system. The overall calibration of the BBR is accomplished by performing a confidence check. The confidence check is conducted by performing a BBR test on a calibrated beam of known modulus. The resulting stiffness values must be within an allowable range or additional calibration steps are required.

After the initial calibration, a verification of calibration procedure is conducted prior to each day of testing. The verification of calibration procedures is an abbreviated variation of the initial load cell, LVDT, and RTD calibration procedures. A confidence check is to be conducted at the beginning of each testing day as well.

\section{Theory of Analysis}

The asphalt beam dimensions were selected such that the elementary BernoulliEuler theory of bending of prismatic beams would be applicable to the analysis of the test results (Anderson, et al., 1994). From the elementary bending theory, the maximum deflection of an elastic prismatic beam in three-point loading occurs at the beam midspan.

The deflection of the beam at midspan, $\delta$, is calculated as:

$$
\text { Midspan Deflection }(\mathrm{mm}) \quad \delta=\frac{\mathrm{PL}^{3}}{48 \mathrm{EI}}
$$


where: $\mathrm{P}=$ applied load $(\mathrm{N})$

$$
\begin{aligned}
& \mathrm{L}=\text { span length }(\mathrm{mm}) \\
& \mathrm{E}=\text { modulus of elasticity }(\mathrm{Pa}) \\
& \mathrm{I}=\text { moment of inertia of section }\left(\mathrm{mm}^{4}\right)
\end{aligned}
$$

The modulus of elasticity, E, can also be defined by the following relationship, where $\mathrm{D}(\mathrm{t})$ is the extensional creep compliance:

$$
\text { Modulus of Elasticity }(\mathrm{Pa}) \quad \mathrm{E}=\frac{1}{\mathrm{D}(\mathrm{t})}
$$

In addition, the inverse of the extensional creep compliance is equivalent to the time dependent creep stiffness, $\mathrm{S}(\mathrm{t})$, of a viscoelastic material. Based upon the elasticviscoelastic correspondence theory, the assumption can be made that the stress distribution in a viscoelastic beam is the same as that in an elastic beam under the same applied load. Thus, the time-dependant strains and displacements of an asphalt specimen can be derived from the elementary bending theory for elastic materials by substituting $\mathrm{S}(\mathrm{t})$ for E (Anderson, et al., 1994):

$$
\text { Midspan Deflection }(\mathrm{mm}) \quad \delta(\mathrm{t})=\frac{\mathrm{PL}^{3}}{48 \mathrm{~S}(\mathrm{t}) \mathrm{I}}
$$

Considering that the moment of inertia, I, is defined as $\mathrm{bh}^{3} / 12$, where (b) and (h) are the width and height of a prismatic beam, respectively, and rearranging to solve for $\mathrm{S}(\mathrm{t})$, the elementary bending theory can take the following final form for evaluating an asphalt specimen:

$$
\text { Creep Stiffness }(\mathrm{Pa}) \quad \mathrm{S}(\mathrm{t})=\frac{\mathrm{PL}^{3}}{4 \mathrm{bh}^{3} \delta(\mathrm{t})}
$$




\section{Thermal Cracking Parameters}

The magnitude of stresses the HMA pavement layer experiences is dependent upon the stiffness of the asphalt binder and its ability to relax those stresses by dissipating energy via permanent flow (Bahia and Anderson, 1995a and Roberts, et al., 1996). The asphalt properties should be such that it flows under stress and have a lower elastic component of response. Thus, parameters where selected that evaluate both the stiffness and its stress relaxation potential.

The creep stiffness parameter, $\mathrm{S}(\mathrm{t})$, was selected to evaluate the extent of thermal stresses that develop in the HMA pavement during thermal contraction. The creep stiffness is characterized by measuring the creep response of asphalts with the BBR at critical temperatures. An HMA pavement composed of a binder with a high creep stiffness has the potential to develop high tensile stresses during thermal contraction.

The logarithmic creep rate, $\mathrm{m}(\mathrm{t})$, is an indicator of the asphalt's ability to relax stresses (Roberts, et al., 1996). The logarithmic creep rate, commonly called the mvalue, is a measure of the rate at which creep stiffness changes with loading time. The $\mathrm{m}$-value is graphically defined as the slope of the log creep stiffness versus log loading time on a master curve, as shown in Figure 2.12 (Roberts, et al., 1996). As the m-value decreases, the rate of relaxation decreases, thus, stresses build as the binder loses its ability to relieve those thermal-induced stresses by permanent flow. 


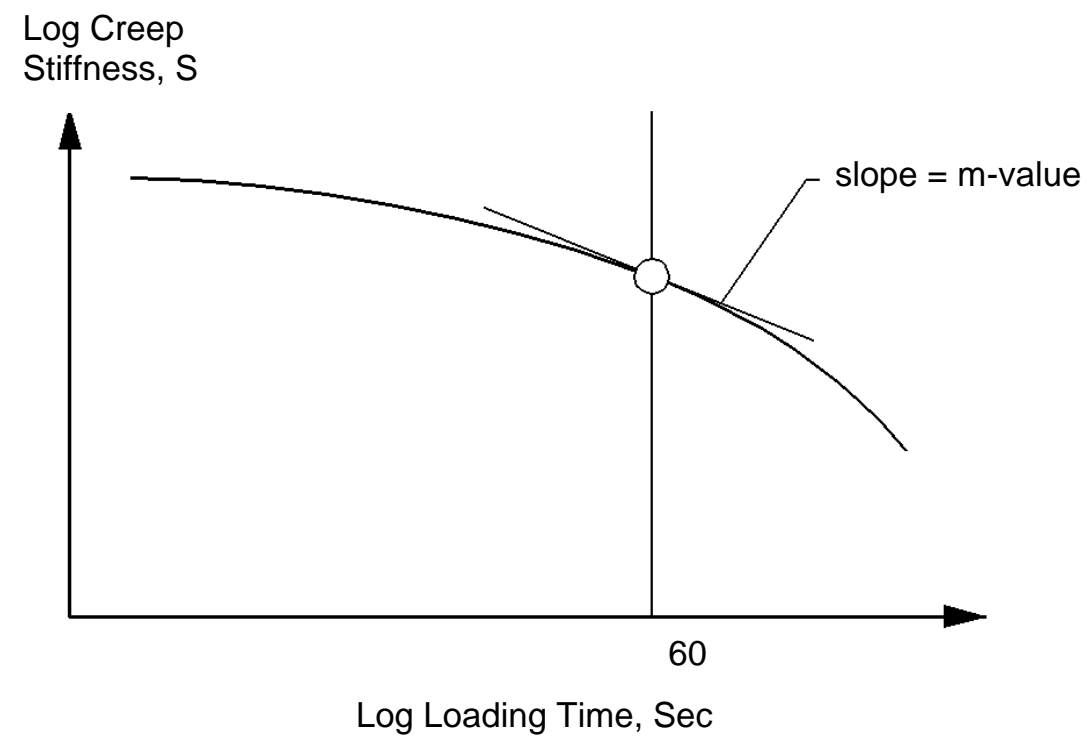

Figure 2.12 Graphical Definition of Creep Rate, m-value

\section{Parameter Specifications}

The work of Bahia and Anderson (1995b) and Roberts, et al. (1996) served as the sources for the following discussion on the BBR test parameter specifications. Both $\mathrm{S}(\mathrm{t})$ and $\mathrm{m}(\mathrm{t})$ are functions of loading time; therefore, a loading time which correlates with thermal cracking was specified. A review of asphalt literature during the SHRP research showed that loading times ranging between 3,600 and 20,000 seconds had been correlated to thermal cracking. Loading times of that duration were not practical for laboratory tests. Therefore, the SHRP researchers used the time-temperature superposition principle to shorten the loading times required to reflect thermal cracking conditions. Using the time-temperature superposition principle allows the response of long loading times to be estimated by testing at higher temperatures for a shorter duration.

To apply the time-temperature superposition principle, creep stiffness versus loading time curves for different temperatures are generated on a log-log plot. A reference temperature is then selected and the curves from the other temperatures are shifted relative to the reference temperature curve until the curves merge into a single 
function, the master curve, as shown in Figure 2.13a (Bahia and Anderson, 1995b). The $\log$ of the shift factors $\left(\mathrm{x}_{1}, \mathrm{x}_{2}\right.$, etc.) needed to overlap the logarithmic curves in Figure 2.13a are also plotted versus temperature, as shown in Figure 2.13b (Bahia and Anderson, 1995b). A temperature shift function is established from the log shift factors versus temperature plot of Figure 2.13b.

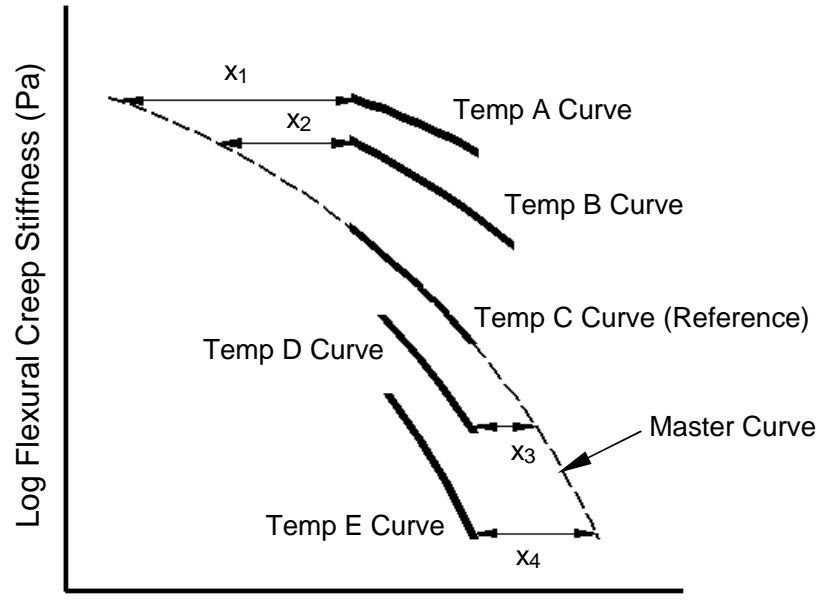

Log Loading Time (sec)

(a)

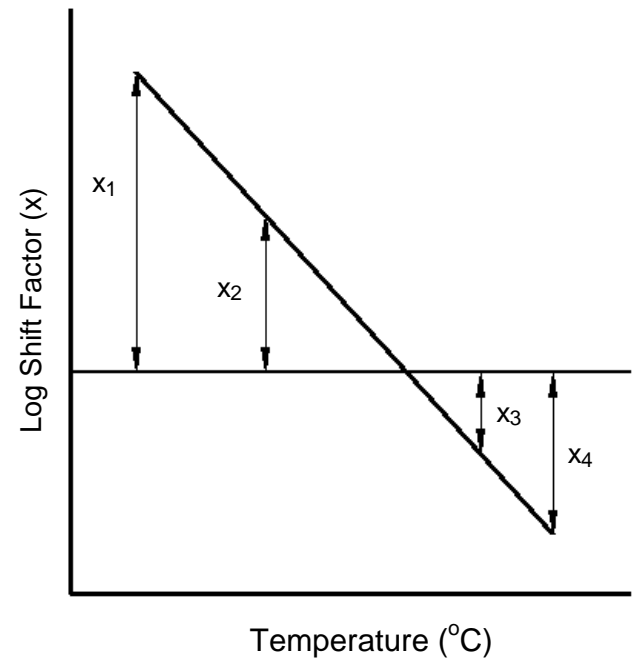

(b)

Figure 2.13 Development of Time-Temperature Shift Factors

It was observed by the SHRP researchers that the shift functions generated from their research were linear with a slope that ranged from only 0.18 to 0.2 log seconds per degree Celsius. The shift function similarities ultimately led to the agreement that a single offset temperature could be used for estimating the binder response at longer loading times from shorter duration tests conducted at higher temperatures. Upon further examination of the shift factors, it was concluded that an offset of $10{ }^{\circ} \mathrm{C}$ above the lowest specification temperature could be used to equate the BBR stiffness at 60 seconds loading time to the asphalt binder stiffness at 7,200 second loading time in the field.

During the BBR test, the creep stiffness and creep rates are determined at six loading times: $8,15,30,60,120$, and 240 seconds. The shape of the log creep stiffness 
versus log loading time master curve, Figure 2.12 and Figure 2.13a, for asphalt binders is determined from the data of the six loading times and the following relationship:

$$
\begin{aligned}
& \log S(t)=A+B \log (t)+C[\log (t)]^{2} \\
& \text { where: } S(t)=\text { asphalt binder stiffness, Pa } \\
& \qquad t=\text { time, sec. }
\end{aligned}
$$

$$
\mathrm{A}, \mathrm{B} \text {, and } \mathrm{C}=\text { constants }
$$

Equation 2-26 and the resultant master creep stiffness curve are used to determine the specification parameters. A maximum limit specification is required for the creep stiffness parameter and a minimum limit specification is required for the $\mathrm{m}$ value parameter. Placing a maximum limit on the creep stiffness restricts the level of stresses that will develop in the HMA pavement. The specification criterion established for creep stiffness at 60 seconds, $\mathrm{S}(60)$, is $300 \mathrm{MPa}$ maximum. The stiffness limit was established based on previous studies that correlated thermal cracking with creep stiffness, as well as additional evaluation tests conducted with the bending beam rheometer by the SHRP researchers.

Placing a minimum limit on the m-value maintains the rate of relaxation such that stresses can be relieved through permanent flow. The specification criterion for the logarithmic rate of relaxation at 60 seconds, $\mathrm{m}(60)$, is 0.300 minimum. The specification was established based on the experience of the Expert Task Group and on data collected from a large number of asphalts tested during SHRP research.

During the SHRP testing, a new hardening behavior was observed in asphalts tested at low temperatures. This phenomenon, termed physical hardening, is a timedependant increase in $\mathrm{S}(\mathrm{t})$ and the decrease in $\mathrm{m}(\mathrm{t})$ which is caused by time-delayed asphalt volume shrinkage. The asphalt hardening was found to increase at a rapid rate within the initial period of isothermal conditioning. Asphalt hardening was observed to increase up to 50 to 100 percent within 24 hours. The physical hardening phenomenon 
was found to be highly asphalt specific and its consequences on asphalt mixtures were observed to be difficult to evaluate. A standard isothermal conditioning time of 60 minutes was observed to be outside the range of rapid hardening, while still being practical for laboratory testing. Therefore, the SHRP researchers established the specification requiring the measurement and reporting of $S(60)$ and $\mathrm{m}(60)$ following 60 minutes of isothermal conditioning in the BBR fluid bath. It was also decided to include the requirement of testing after 24 hours of isothermal conditioning to provide an indication of the potential of the binder for physical hardening; however, the report of $\mathrm{S}(60)$ and $\mathrm{m}(60)$ following 24 hours of isothermal conditioning is for information purposes only.

\subsection{Summary}

The performance grading system is a definite improvement over the previous asphalt binder grading systems. Engineering principles are now used to relate measured physical properties to field performance. A complete range of temperatures is now considered. A constant criterion is used for all PG grades, with the temperature at which the asphalt properties changing in consideration of the asphalt grade for the climate, traffic loadings and speed.

New and revised conditioning procedures enable the performance of asphalt binders to be evaluated all three critical stages: the transporting and handling of original asphalt binder before mixing, the short-term aging of binders following HMA production and construction, and the continued long-term aging during the pavement service life.

Performance parameters are in place to evaluate a binder's resistance to aging during construction and during its service life. The parameters ensure that the binder is workable during the mixing and placement of HMA pavements. Performance parameters also control rutting, fatigue cracking and thermal cracking pavement distresses. 


\section{Chapter 3}

\section{Research Approach}

\subsection{Introduction}

The objective of this research was a straightforward evaluation of the performance grading equipment at the Asphalt Technology Laboratory and the related testing protocol. However, due to the newness of both the test protocol and equipment, accomplishing this objective was fraught with difficulties and complication. The test protocols and specifications are under constant scrutiny, review, and occasional revision. To make this problem manageable, it was decided to use the test procedures in force when the research was initiated. Training in the proper methods required a considerable effort. Calibration and verification of the equipment was time consuming. In some cases, supplemental equipment and tools were developed to assist with test performance. Once confidence was developed in conducting the tests and in the equipment performance, a test program was developed. Since the emphasis of the research was on the equipment and testing protocol, a single grade and source of asphalt was used for all tests.

\subsection{Testing Program Preparation}

\section{Operator Training}

A thorough review of the equipment manufacturer's documentation, related SHRP documentation and the AASHTO standard specifications test methods was first conducted to establish a full understanding of the equipment's scope of use, limitations, and testing protocol and procedures. A trial period of testing was conducted to learn the testing protocol and procedures and to become proficient in asphalt sample preparation and handling, unit calibration and operation, and testing technique via hands-on experience. The trial testing period was also intended to provide the opportunity to identify and document potential sources of equipment and user error. 
A one-day training workshop provided by Applied Test Systems, Inc. (ATS) for the pressure aging vessel and bending beam rheometer was attended. The workshop provided instruction on equipment calibration, operation, maintenance, and sample specimen preparation. As a result of difficulties experienced with the DSR during the trial testing period, Dennis Burke, a senior technician for the Citgo Asphalt Refining Company and an experienced operator of the Bohlin DSR and Brookfield RV, graciously visited the WVU Asphalt Technology Laboratory to evaluate the performance of the DSR. Mr. Burke provided instruction on sample specimen preparation and equipment calibration/operation of the RV and DSR.

\section{Equipment Preparation}

The majority of the hardware and accessories required for the operation of each unit (i.e. sample specimen molds, standard viscosity calibration fluid, etc.) was available from the equipment manufacturer or by another asphalt industry supplier. There were some accessories that were not available from either of the above and therefore had to be fabricated.

A tool for scraping RTFO-aged asphalt residue from the RTFO specimen bottles was fabricated. Two different styles of tools were developed in an effort to achieve the AASHTO T240 requirement of 90 percent residue removal from the specimen bottles.

One tool was designed such that the residue was removed by scraping down the longitudinal length of the specimen bottle. The other style of tool removed the residue by scraping around the periphery of the bottle. The later tool design was more efficient than the former; however, the required 90 percent removal criterion could never be achieved with either style of scraping tool.

A DSR specimen trimming tool was not available from a commercial supplier; therefore, a trimming tool was fabricated. The tool was designed such that the asphalt specimen was trimmed by dragging the edge of the tool around the periphery of the parallel plates. The trimming tool design was evaluated via multiple trial tests and worked well. Therefore, the tool was used for the testing program. 
The calibration of each unit was verified and recalibrated as necessary. The flow rate of the RTFO was verified with a flow meter and the oven temperature was verified with a NIST traceable ASTM Loss of Heat Thermometer $13{ }^{\circ} \mathrm{C}$. The pressure and temperature calibration of the pressure aging vessel is to be conducted by a commercial calibration service provider. The PAV calibration was not verified prior to the testing program. Mr. Burke conducted the RV temperature controller and rotary transducer calibration verification. The temperature controller was verified using an ohmmeter and a NIST traceable thermister. The rotary transducer was verified using Brookfield Viscosity Standard Fluid, No. HT100000. Mr. Burke also verified the calibration of the dynamic shear rheometer. The temperature controller was verified using a Fluke 77III multimeter and a Cannon wafer thermister, No. 9728-V95. The overall calibration of the unit was verified using Cannon Viscosity Standard Fluid, No. N2700000SP. The bending beam rheometer LVDT and load cell was calibrated using the standard weights, thickness gauge, and confidence beam supplied by ATS. The ATS technical representative stated that the resistance thermal detector on their units do not require calibration; therefore, it was not calibrated.

The equipment calibration of each unit was checked multiple times at random during the trial period in order to maintain a level of confidence in the stability of the equipment. Multiple trial tests were conducted until consistency in results was established.

\section{Testing Protocol}

The AASHTO standard specifications were the testing protocol source for each unit. The protocol for the selection of the rotational viscometer spindle type and spindle speed, however, was not straightforward. The AASHTO TP48 test method requires that a test first be conducted at 20 RPM. If the resultant torque is out of range for the selected spindle and speed, then the operator is to change the spindle or speed as per the manufacturer's recommendations. The specifications, however, are silent regarding the spindle types. In addition, the precision and bias criterion of the AASHTO test method is established only for $135{ }^{\circ} \mathrm{C}$ test temperatures; no criterion is stated for higher test 
temperatures that are encountered when performing equiviscosity tests.

The AASHTO TP48 protocol was compared to AASHTO MP1, and SHRP and Asphalt Institute literature for additional direction with regard to spindle type selection. The ASTM D4402 protocol was referenced in AASHTO MP1. The ASTM D4402 states that testing with more than one spindle might be required to properly select the appropriate spindle type. The ASTM standard also states that testing should start at 20 RPM for the Brookfield RV, HA, and HB series and 12 RPM for the LV series. The standard states that changes to spindle speed or spindle type may be required if viscosity readings are not within a specified range. No recommendations are presented in the specifications for a particular spindle type. A SC4-27 spindle is recommended in the SHRP literature (Petersen, et al., 1994b). A 20-RPM operating speed is also recommended by the SHRP researchers in a different volume of the final report (Anderson, et al., 1994). However, neither volume of the SHRP final report contains documentation to which Brookfield series (LV, RV, HA, or HB) their spindle and speed recommendations apply.

The Asphalt Institute recommends conducting viscosity tests at $135{ }^{\circ} \mathrm{C}$ and $165{ }^{\circ} \mathrm{C}$ when establishing temperature-viscosity curves (Asphalt Institute, 2001). However, the Asphalt Institute literature provided no direction on what spindle types or speeds to use when conducting the tests.

The rotational viscometer testing protocol seemed ambiguous when comparing the direction provided by AASHTO, ASTM, SHRP, and the Asphalt Institute. Therefore, the decision was made to follow the AASHTO TP48 protocol, with the exception that the SC4-27 spindle was used with an operating speed of 20 RPM, as per the SHRP literature. Additionally, the testing program would include tests to temperatures of $165^{\circ} \mathrm{C}$, per Asphalt Institute methodology. The AASHTO standard specifications were, however, strictly followed for every other test. 


\subsection{Testing Program}

A graduate student was employed for conducting the testing program after the testing preparation procedures described in the previous section were completed. The student was trained on the equipment operation and sample preparation. The student also performed multiple trial tests with each unit until a consistency in test results was established.

Once the objectives of the trial testing period were accomplished, the actual testing program was initiated to evaluate the equipment precision and repeatability. Rotational viscometer, dynamic shear rheometer, and bending beam rheometer tests were conducted on PG 70-22 asphalt. The tests and conditioning procedures are summarized in Table 3.1.

The PG 70-22 neat asphalt underwent rolling thin film oven and pressure aging vessel conditioning procedures, where required. Asphalt sample sets were created. The sample sets consisted of neat, RTFO-aged, and PAV-aged asphalt stored in three-ounce tins. Each three-ounce tin from a sample set was used to make a test specimen for a specific test. One test specimen, either a rotational viscometer, dynamic shear rheometer, or bending beam rheometer test specimen, was made from each three-ounce tin. A summary of the testing program is provided in Figure 3.1 and Figure 3.2. 


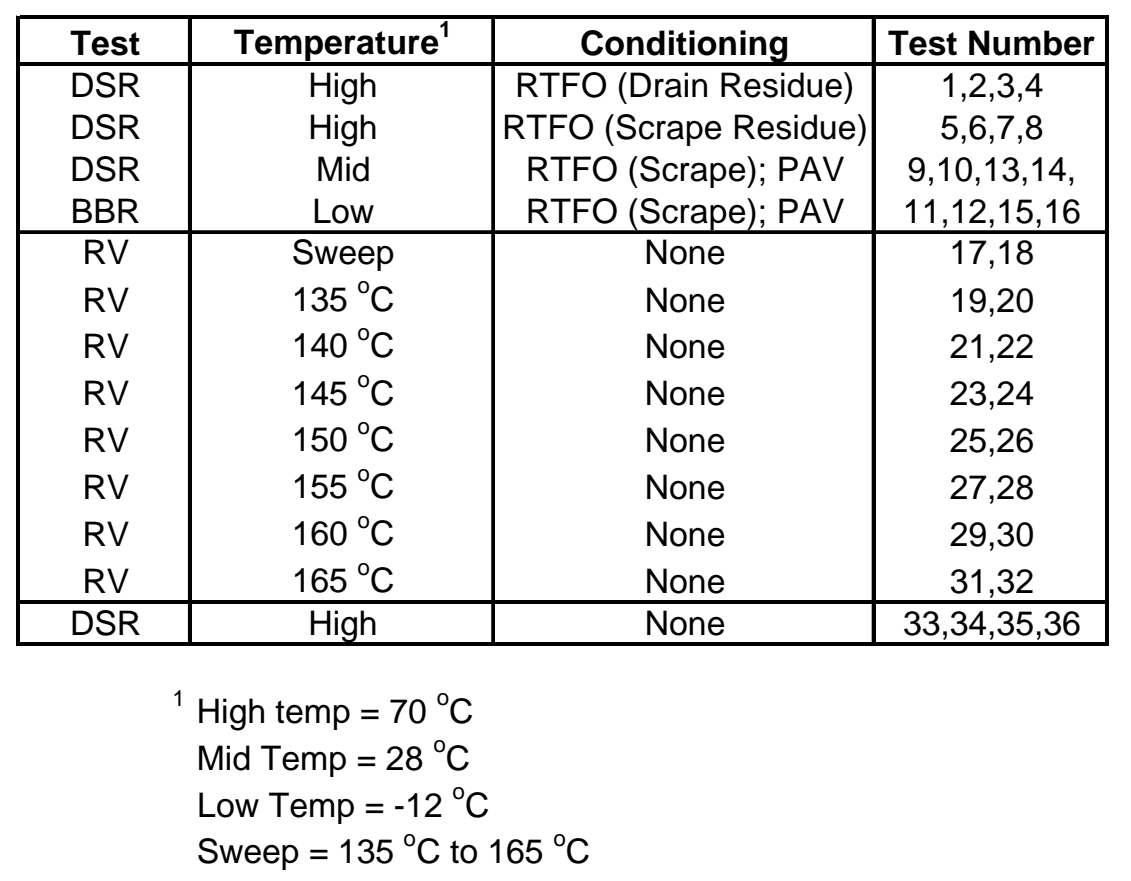

Table 3.1 Testing and Conditioning Sequence 


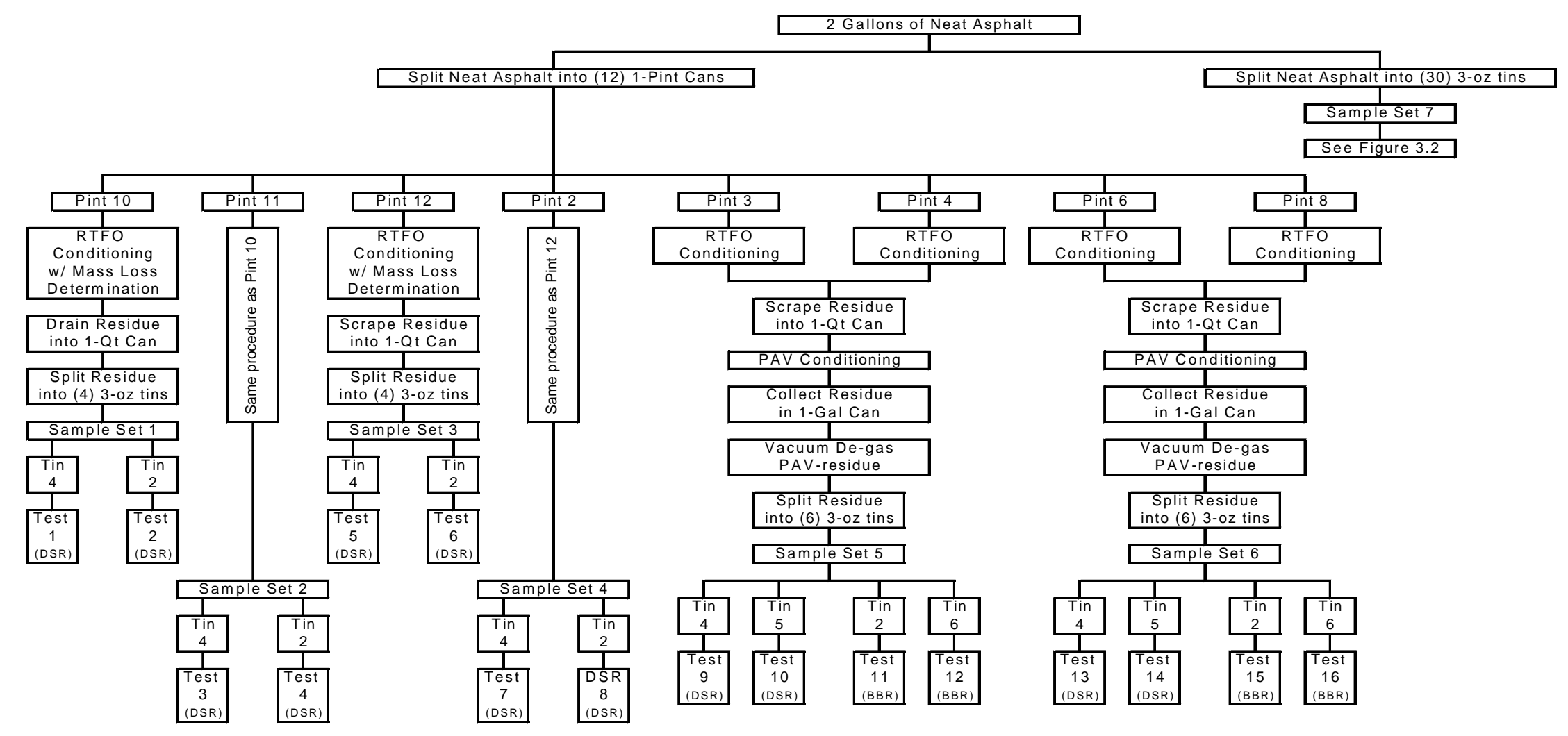

Figure 3.1 Testing Program Summary 


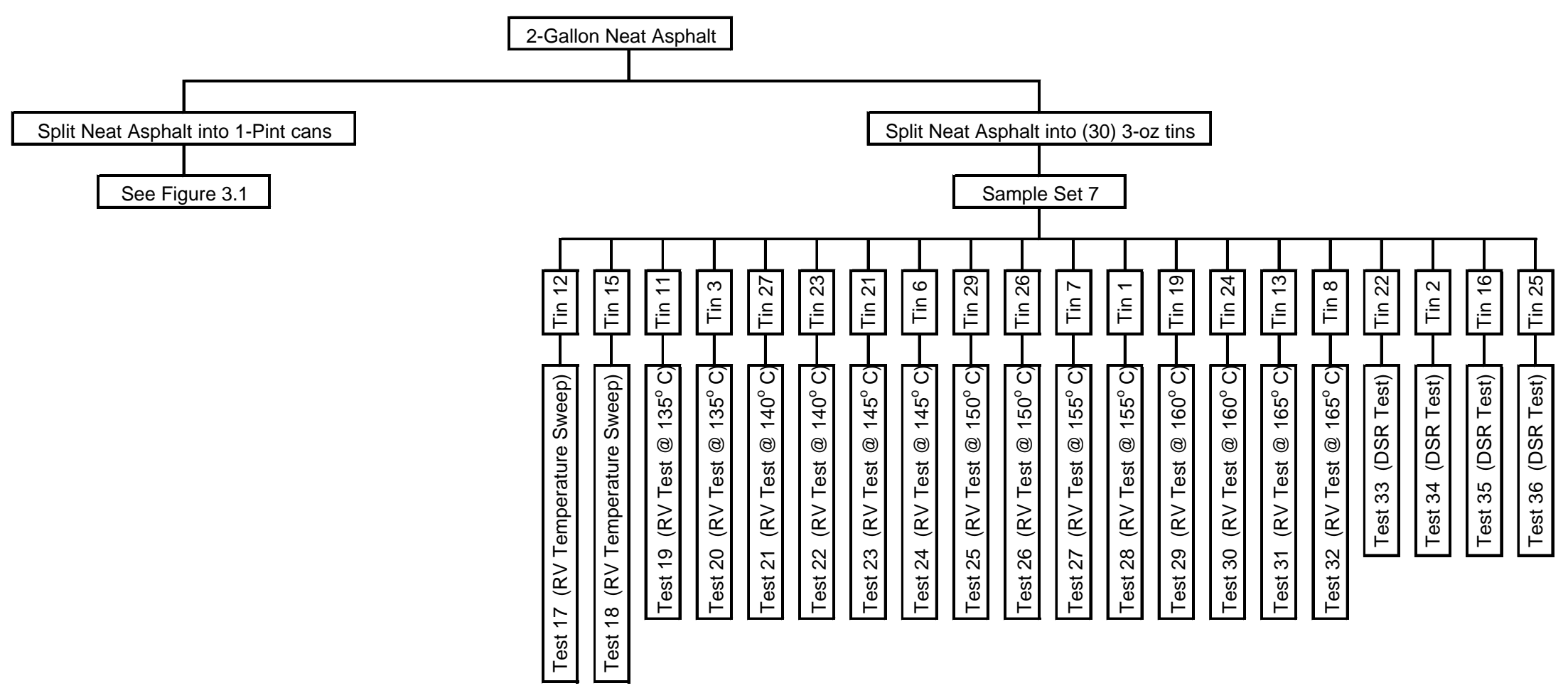

Figure 3.2 Testing Program Summary - continued 
The testing program required seven sample sets:

\begin{tabular}{|c|c|c|}
\hline Sample Set & $\underline{\text { Test }}$ & Conditioning \\
\hline 1 & DSR & RTFO (Drain) \\
\hline 2 & Replicate of Sample Set 1 & \\
\hline 3 & DSR & RTFO (Scrape) \\
\hline 4 & Replicate of Sample Set 3 & \\
\hline 5 & DSR; BBR & RTFO (Scrape); PAV \\
\hline 6 & Replicate of Sample Set 5 & \\
\hline 7 & RV; DSR & None \\
\hline
\end{tabular}

Sample Sets 1 and 2 are replicates. Each set required one-pint of binder that was conditioned in the RTFO and then split into sample tins for testing with the DSR. The RTFO conditioning bottles were drained, rather than scraping, to remove the binder following conditioning.

Sample Sets 3 and 4 are replicates. The laboratory protocol was similar to Sets 1 and 2, with the exception of scraping the RTFO-aged material following conditioning rather than draining.

Sample Sets 5 and 6 are replicates. Each set required two pints of binder. The samples were conditioned in the RTFO. The RTFO-aged material was scraped from the bottles following conditioning. The RTFO-aged material was then conditioned in the PAV and vacuum degassed. The PAV-aged material was split into sample tins for testing with the DSR and BBR.

Sample Set 7 was used for RV testing and DSR tests on neat asphalt; therefore, no conditioning procedures were conducted prior to testing. Two replicate tests were performed using a RV temperature sweep procedure. Seven pairs of test tins were used for replicate single temperature tests. The remaining tins were used for DSR tests. 
Two gallons of PG 70-22 neat asphalt binder was required for the testing program. The gallon containers of neat asphalt were heated in a $135^{\circ} \mathrm{C}$ oven once and immediately split into 6 one-pint cans and 15 three-ounce tins. Approximately 350 grams of the neat asphalt was poured into each one-pint can and approximately 40 grams of the neat asphalt was poured into each three-ounce tin. The filled cans and tins were covered and stored at room temperature. Thus, 12 one-pint cans and 30 three-ounce tins were available for further conditioning and testing.

As shown in Figures 3.1 and 3.2, only 8 one-pint cans and 20 three-ounce tins of neat asphalt were required for the testing program; the additional 4 one-pint cans and 10 three-ounce tins served as a reserve supply in case problems were encountered during the conditioning and testing procedures. The pints and tins used for testing program and for sample reserves were randomly selected.

The neat asphalt from each of the 8 one-pint cans was conditioned with the RTFO, as per AASHTO T240. Each of the one-pint cans was heated in a $135^{\circ} \mathrm{C}$ oven until sufficiently fluid to pour into the RTFO bottles. A mass loss test was conducted with the RTFO conditioning procedures for Sample Sets 1 through 4. The asphalt residue from each RTFO conditioning procedure was collected in a one-quart can. The residue from the RTFO bottles was collected by two different methods. One method was to allow the residue to drain from the RTFO bottles. The residue from the RTFO conditioning procedures for Sample Sets 1 and 2 was collected in this manner. The residue from the remaining RTFO conditioning procedures was collected by scraping the residue from the RTFO bottles. The one-pint cans containing the RTFO residue was covered and stored at room temperature for further conditioning and testing. DSR tests were later conducted on both the drained RTFO residue and scraped RTFO residue to determine if scraping yields significant differences in binder properties.

The residue collected from the RTFO conditioning procedures for Sample Sets 5 and 6 underwent PAV conditioning, as per AASHTO PP1. The PAV residue for each sample set was collected in a one-gallon can and then degassed in a vacuum oven as 
specified in AASHTO PP1. Each can of PAV-aged residue was covered and stored at room temperature for future testing.

Each of Sample Sets 1 through 4 consisted of 4 three-ounce tins containing approximately 40 grams of the RTFO aged asphalt residue. The one-quart cans of RTFO residue were heated in a $135{ }^{\circ} \mathrm{C}$ oven until sufficiently fluid to pour into the tins. The three-ounce tins were covered and stored at room temperature. Only 2 of the 4 tins in each sample set were used for testing. The remaining 2 tins served as a spare tins in case problems were encountered during testing. As shown in Figure 3.1, the residue stored in the three-ounce tins was used for DSR testing, as per AASHTO TP5. Only one DSR test specimen was made from each three-ounce tin. Each DSR specimen was made using a silicone specimen mold. The RTFO residue from each three-ounce tin was heated in a $135{ }^{\circ} \mathrm{C}$ oven until sufficiently fluid to pour into the silicone specimen mold.

Each of Sample Sets 5 and 6 consisted of 6 three-ounce tins containing approximately 40 grams of the PAV-aged asphalt residue. The one-gallon cans of PAV residue were heated in a $163{ }^{\circ} \mathrm{C}$ oven until sufficiently fluid to pour into the tins. The three-ounce tins were covered and stored at room temperature. Only 4 of the 6 tins in each sample set were used for testing. The remaining 2 tins served as a spare samples. The PAV residue stored in the three-ounce tins were used for DSR testing, as per AASHTO TP5, and BBR testing, as per AASHTO TP1. Only one DSR or BBR test specimen was made from each three-ounce tin. The DSR specimens were made using a silicone mold and the BBR specimens were made using aluminum bar molds.

Sample Set 7 was made during the initial splitting of the 2 gallons of asphalt. Approximately 40 grams of neat asphalt was poured into 30 three-ounce tins. Only 20 of the 30 tins were used for testing; the remaining 10 tins served as spares.

As shown in Figure 3.2, the neat asphalt in each three-ounce tin was used for RV and DSR testing. RV tests were conducted with 16 of the specimen tins in Sample Set 7 while DSR tests were conducted on the remaining tins. Only one RV specimen or DSR 
specimen was made from each three-ounce tin. Each tin was heated in a $135^{\circ} \mathrm{C}$ oven until sufficiently fluid to pour into the RV sample holder or the DSR silicone specimen mold. The DSR and RV tests were conducted as per AASHTO TP5 and AASHTO TP48, respectively.

As shown in Figure 3.2, two duplicate RV temperature sweep tests were conducted. Each temperature sweep test was performed on a single test specimen. Viscosity measurements were taken in $5{ }^{\circ} \mathrm{C}$ increments beginning at $135{ }^{\circ} \mathrm{C}$ up to $165{ }^{\circ} \mathrm{C}$. A 10-minute equilibrium period at each test temperature was allowed before the viscosity measurements were taken. Three viscosity measurements were taken at 1-minute intervals at each $5{ }^{\circ} \mathrm{C}$ incremental temperature. Duplicate single temperature RV tests were also performed at temperatures ranging from $135{ }^{\circ} \mathrm{C}$ to $165^{\circ} \mathrm{C}$, at $5{ }^{\circ} \mathrm{C}$ intervals, as shown in Figure 3.2.

The sample set conditioning sequence is shown is Table 3.2. The sample set conditioning sequence, the pints conditioned for each sample set, the selection order of replacement tins, the selection order of tins for testing, the selection order of replacement tins, and the tests conducted from each sample set were randomly selected. The testing sequence is shown in Table 3.3. The testing order was randomly selected as well.

\begin{tabular}{|c|c|c|c|c|c|c|}
\hline $\begin{array}{c}\text { Sample } \\
\text { Set }^{1}\end{array}$ & $\begin{array}{c}\text { Pint No.'s } \\
\text { Conditioned } \\
\text { for Sample } \\
\text { Set }\end{array}$ & $\begin{array}{c}\text { Selection } \\
\text { Order of } \\
\text { Replacement } \\
\text { Pints }\end{array}$ & $\begin{array}{c}\text { No. of } \\
\text { Tins in } \\
\text { Sample } \\
\text { Set }\end{array}$ & $\begin{array}{c}\text { Selection } \\
\text { Order of } \\
\text { Tins for } \\
\text { Testing }\end{array}$ & $\begin{array}{c}\text { Selection } \\
\text { Order of } \\
\text { Replacement } \\
\text { Tins }\end{array}$ & $\begin{array}{c}\text { Tests } \\
\text { Conducted } \\
\text { from Sample } \\
\text { Set }\end{array}$ \\
\hline 4 & 2 & $9-5-7-1$ & 4 & $4-2$ & $1-3$ & 7,8 \\
\hline 5 & 3,4 & $9-5-7-1$ & 4 & $4-5-2-6$ & $1-3$ & $9,10,11,12$ \\
\hline 1 & 10 & $9-5-7-1$ & 4 & $4-2$ & $1-3$ & 1,2 \\
\hline 2 & 11 & $9-5-7-1$ & 4 & $4-2$ & $1-3$ & 3,4 \\
\hline 6 & 6,8 & $9-5-7-1$ & 6 & $4-5-2-6$ & $1-3$ & $13,14,15,16$ \\
\hline 3 & 12 & $9-5-7-1$ & 6 & $4-2$ & $1-3$ & 5,6 \\
\hline
\end{tabular}

Listed in order of conditioning sequence

Table 3.2 Sample Set Conditioning Sequence 


\begin{tabular}{|c|c|c|c|c|}
\hline Test No. ${ }^{1}$ & Test Description & Sample Set & Tin No. & $\begin{array}{l}\text { Selection Order of } \\
\text { Replacement Tins }\end{array}$ \\
\hline 33 & DSR (Neat) & 7 & 22 & $10-18-9-5-30-14-20-28-17-4$ \\
\hline 34 & DSR (Neat) & 7 & 2 & $10-18-9-5-30-14-20-28-17-4$ \\
\hline 27 & $\mathrm{RV}-155^{\circ} \mathrm{C}$ & 7 & 7 & $10-18-9-5-30-14-20-28-17-4$ \\
\hline 12 & BBR & 5 & 6 & $1-3$ \\
\hline 29 & $\mathrm{RV}-160^{\circ} \mathrm{C}$ & 7 & 19 & $10-18-9-5-30-14-20-28-17-4$ \\
\hline 28 & $\mathrm{RV}-155^{\circ} \mathrm{C}$ & 7 & 1 & $10-18-9-5-30-14-20-28-17-4$ \\
\hline 8 & DSR (RTFO) & 4 & 2 & $1-3$ \\
\hline 7 & DSR (RTFO) & 4 & 4 & $1-3$ \\
\hline 32 & $\mathrm{RV}-165^{\circ} \mathrm{C}$ & 7 & 8 & $10-18-9-5-30-14-20-28-17-4$ \\
\hline 23 & $\mathrm{RV}-145^{\circ} \mathrm{C}$ & 7 & 21 & $10-18-9-5-30-14-20-28-17-4$ \\
\hline 24 & $\mathrm{RV}-145^{\circ} \mathrm{C}$ & 7 & 6 & $10-18-9-5-30-14-20-28-17-4$ \\
\hline 19 & $\mathrm{RV}-135^{\circ} \mathrm{C}$ & 7 & 11 & $10-18-9-5-30-14-20-28-17-4$ \\
\hline 18 & RV - Temp. Sweep & 7 & 15 & 10-18-9-5-30-14-20-28-17-4 \\
\hline 26 & $\mathrm{RV}-150^{\circ} \mathrm{C}$ & 7 & 26 & $10-18-9-5-30-14-20-28-17-4$ \\
\hline 20 & $\mathrm{RV}-135^{\circ} \mathrm{C}$ & 7 & 3 & $10-18-9-5-30-14-20-28-17-4$ \\
\hline 36 & DSR (Neat) & 7 & 25 & 10-18-9-5-30-14-20-28-17-4 \\
\hline 10 & DSR (PAV) & 5 & 5 & $1-3$ \\
\hline 6 & DSR (RTFO) & 3 & 2 & $1-3$ \\
\hline 14 & DSR (PAV) & 6 & 5 & $1-3$ \\
\hline 35 & DSR (Neat) & 7 & 16 & $10-18-9-5-30-14-20-28-17-4$ \\
\hline 15 & BBR & 6 & 2 & $1-3$ \\
\hline 2 & DSR (RTFO) & 1 & 2 & $1-3$ \\
\hline 3 & DSR (RTFO) & 2 & 4 & $1-3$ \\
\hline 5 & DSR (RTFO) & 3 & 4 & $1-3$ \\
\hline 21 & $\mathrm{RV}-140^{\circ} \mathrm{C}$ & 7 & 27 & $10-18-9-5-30-14-20-28-17-4$ \\
\hline 11 & BBR & 5 & 2 & $1-3$ \\
\hline 30 & $\mathrm{RV}-160^{\circ} \mathrm{C}$ & 7 & 24 & $10-18-9-5-30-14-20-28-17-4$ \\
\hline 31 & $\mathrm{RV}-165^{\circ} \mathrm{C}$ & 7 & 13 & $10-18-9-5-30-14-20-28-17-4$ \\
\hline 1 & DSR (RTFO) & 1 & 4 & $1-3$ \\
\hline 16 & BBR & 6 & 6 & $1-3$ \\
\hline 4 & DSR (RTFO) & 2 & 2 & $1-3$ \\
\hline 22 & $\mathrm{RV}-140^{\circ} \mathrm{C}$ & 7 & 23 & $10-18-9-5-30-14-20-28-17-4$ \\
\hline 9 & DSR (PAV) & 5 & 4 & $1-3$ \\
\hline 17 & RV - Temp. Sweep & 7 & 12 & $10-18-9-5-30-14-20-28-17-4$ \\
\hline 25 & $\mathrm{RV}-150^{\circ} \mathrm{C}$ & 7 & 29 & $10-18-9-5-30-14-20-28-17-4$ \\
\hline 13 & DSR (PAV) & 6 & 4 & $1-3$ \\
\hline
\end{tabular}

${ }^{1}$ Listed in order of testing sequence. The test number is defined in Figures 3.1 and 3.2.

Table 3.3 Testing Sequence 
Chapter 4

\section{Test Results Analysis and Equipment Evaluation}

\subsection{Introduction}

The purpose of this chapter is to provide an analysis of the test results. The test results are used to draw conclusions on the performance of the SHRP testing equipment. The test data were compiled and summarized to characterize the equipment's ability to meet the SHRP performance test specifications and the AASHTO precision criteria. A discussion of potential causes for test errors was prepared. This chapter also includes a discussion on the time and effort that was required to set up and calibrate the equipment and to perform trial tests such that a confidence in the equipment operation was achieved.

\subsection{Rolling Thin Film Oven}

\section{Testing Results Analysis}

The average mass loss for each RTFO test is presented in Table 4.1. Precision of mass loss test criteria was not available in AASHTO T240 at the time of this research; therefore, no analysis of the results was conducted.

\begin{tabular}{|c|c|c|c|c|c|c|}
\hline $\begin{array}{l}\text { Sample } \\
\text { Set }\end{array}$ & Pint No. & $\begin{array}{c}\text { RTFO } \\
\text { Bottle No. }\end{array}$ & $\begin{array}{c}\text { Pre- } \\
\text { Conditioned } \\
\text { Mass } \\
\text { (grams) } \\
\end{array}$ & $\begin{array}{c}\text { Post- } \\
\text { Conditioned } \\
\text { Mass } \\
\text { (grams) } \\
\end{array}$ & $\begin{array}{c}\text { Mass Loss } \\
\text { (grams) }\end{array}$ & $\begin{array}{c}\text { Average } \\
\text { Mass Loss } \\
(\%)\end{array}$ \\
\hline \multirow{2}{*}{1} & \multirow{2}{*}{10} & Bottle 1 & 34.992 & 34.821 & 0.00489 & \multirow{2}{*}{0.3069} \\
\hline & & Bottle 2 & 35.056 & 35.012 & 0.00126 & \\
\hline \multirow{2}{*}{2} & \multirow{2}{*}{11} & Bottle 1 & 35.399 & 35.387 & 0.00034 & \multirow{2}{*}{0.0411} \\
\hline & & Bottle 2 & 35.211 & 35.194 & 0.00048 & \\
\hline \multirow{2}{*}{3} & \multirow{2}{*}{12} & Bottle 1 & 34.826 & 34.796 & 0.00086 & \multirow{2}{*}{0.1064} \\
\hline & & Bottle 2 & 34.713 & 34.669 & 0.00127 & \\
\hline \multirow[b]{2}{*}{4} & \multirow[b]{2}{*}{$y$} & Bottle 1 & 34.852 & 34.800 & 0.00149 & \multirow{2}{*}{0.0933} \\
\hline & & Bottle 2 & 34.785 & 34.772 & 0.00037 & \\
\hline
\end{tabular}

Table 4.1 RTFO Mass Loss Results 


\section{Equipment Evaluation}

The Despatch RTFO was simple in operation and required minimal time to become proficient in its use. The procedures outlined in the AASHTO T240 test method for the neat asphalt heating and handling during the preparation of the sample specimens are very basic. There is little potential for user error when conducting the conditioning procedure as per the test method.

The only notable difficulty experienced during the testing was collecting the RTFO residue from the bottles. The AASHTO specifications require that if the RTFO residue is removed from the bottles via scraping, rather than allowing the residue to drain, at least $90 \%$ of the residue must be collected from each bottle. Each of the eight RTFO bottles are filled with 35 grams of neat asphalt prior to conditioning; therefore, 31.5 grams of the RTFO residue must be collected from each bottle when scraping, as per AASHTO specifications. No minimum amount to be collected is specified when allowing the RTFO-aged asphalt to free drain.

Collecting $90 \%$ of the RTFO residue by scraping proved to be very difficult. Several different scraping tools and techniques were tried prior to the start of the testing program; however, none of the attempts were successful in achieving the required amount of residue from each bottle. The average amount of RTFO residue that was able to be collected from each bottle by draining was approximately 24 grams, or 69 percent. The average amount of RTFO residue collected from each bottle by scraping was approximately 28 grams, or 80 percent.

The decision was made to proceed with the testing program and to note any differences observed between the DSR tests conducted from drained RTFO residue and from scraped RTFO residue. No significant differences were observed in the DSR test results on residue collected by the different methods. 


\subsection{Pressure Aging Vessel}

\section{Testing Results Analysis}

The pressure aging vessel conditioning procedure yields no data to be evaluated.

\section{Equipment Evaluation}

The ATS pressure aging vessel and the NAPCO vacuum oven operation requires minimal user input. Once the asphalt specimens are placed in the PAV, the unit software controls the conditioning procedure. The operator must follow the AASHTO PP1 procedures for the RTFO-aged asphalt sample preparation as well as the vacuum oven degassing procedures following the PAV conditioning. When these procedures are followed, the potential for user error is minimal.

The ATS PAV consistently maintained the temperature and pressure range requirements during conditioning, as per AASHTO criteria. Likewise, the NAPCO vacuum oven functioned properly during the testing program. The vacuum oven maintained the temperature and pressure during degassing, as per AASHTO precision criteria.

\subsection{Rotational Viscometer}

\section{Testing Results Analysis}

Summaries of the test results for duplicate rotational viscometer temperature sweep Tests 17 and 18 are shown in Table 4.2 and Table 4.3. The test results for single temperature rotational viscometer Tests 19 through 25 are summarized in Table 4.4. The test results in these tables are shown in SI units, with viscosity and shear stress expressed as milliPascal-seconds (mPa.s) and Newtons per square meter $\left(\mathrm{N} / \mathrm{m}^{2}\right)$, respectively. 


\begin{tabular}{|c|c|c|c|c|c|c|c|}
\hline $\begin{array}{c}\text { Test } \\
\text { Temperature } \\
\left({ }^{\circ} \mathrm{C}\right)\end{array}$ & $\begin{array}{c}\text { Actual } \\
\text { Temperature } \\
\left({ }^{\circ} \mathrm{C}\right)\end{array}$ & $\begin{array}{l}\text { Speed } \\
\text { (RPM) }\end{array}$ & $\begin{array}{c}\text { Torque } \\
\text { (\%) }\end{array}$ & $\begin{array}{c}\text { Shear Stress } \\
\qquad\left(\mathrm{N} / \mathrm{m}^{2}\right)\end{array}$ & $\begin{array}{c}\text { Shear Rate } \\
\left(\sec ^{-1}\right)\end{array}$ & $\begin{array}{c}\text { Viscosity } \\
\text { (mPa.s) }\end{array}$ & $\begin{array}{c}\text { Average } \\
\text { Viscosity } \\
\text { (mPa.s) }\end{array}$ \\
\hline 135 & 135.10 & 20.00 & 3.86 & 33.15 & 6.80 & 487.50 & \\
\hline 135 & 135.10 & 20.00 & 3.83 & 32.30 & 6.80 & 475.00 & 479.2 \\
\hline 135 & 134.90 & 20.00 & 3.83 & 32.30 & 6.80 & 475.00 & \\
\hline 140 & 140.10 & 20.00 & 2.68 & 22.95 & 6.80 & 337.50 & \\
\hline 140 & 140.10 & 20.00 & 2.66 & 22.95 & 6.80 & 337.50 & 337.5 \\
\hline 140 & 139.90 & 20.00 & 2.69 & 22.95 & 6.80 & 337.50 & \\
\hline 145 & 145.20 & 20.00 & 1.92 & 16.15 & 6.80 & 237.50 & \\
\hline 145 & 145.10 & 20.00 & 1.92 & 16.15 & 6.80 & 237.50 & 237.5 \\
\hline 145 & 144.80 & 20.00 & 1.91 & 16.15 & 6.80 & 237.50 & \\
\hline 150 & 150.20 & 20.00 & 1.37 & 11.90 & 6.80 & 175.00 & \\
\hline 150 & 149.90 & 20.00 & 1.34 & 11.05 & 6.80 & 162.50 & 166.7 \\
\hline 150 & 149.80 & 20.00 & 1.32 & 11.05 & 6.80 & 162.50 & \\
\hline 155 & 155.20 & 20.00 & 0.92 & 7.65 & 6.80 & 112.50 & \\
\hline 155 & 155.20 & 20.00 & 0.90 & 7.65 & 6.80 & 112.50 & 112.5 \\
\hline 155 & 155.10 & 20.00 & 0.89 & 7.65 & 6.80 & 112.50 & \\
\hline 160 & 160.10 & 20.00 & 0.58 & 5.10 & 6.80 & 75.00 & \\
\hline 160 & 159.90 & 20.00 & 0.55 & 5.10 & 6.80 & 75.00 & 75.00 \\
\hline 160 & 159.80 & 20.00 & 0.55 & 5.10 & 6.80 & 75.00 & \\
\hline 165 & 165.00 & 20.00 & 0.31 & 2.55 & 6.80 & 37.50 & \\
\hline 165 & 164.80 & 20.00 & 0.33 & 2.55 & 6.80 & 37.50 & 37.50 \\
\hline 165 & 165.10 & 20.00 & 0.31 & 2.55 & 6.80 & 37.50 & \\
\hline
\end{tabular}

Table 4.2 RV Temperature Sweep Test 17 Results

\begin{tabular}{|c|c|c|c|c|c|c|c|}
\hline $\begin{array}{c}\text { Test } \\
\text { Temperature } \\
\left({ }^{\circ} \mathrm{C}\right)\end{array}$ & $\begin{array}{c}\text { Actual } \\
\text { Temperature } \\
\left({ }^{\circ} \mathrm{C}\right)\end{array}$ & $\begin{array}{l}\text { Speed } \\
\text { (RPM) }\end{array}$ & $\begin{array}{c}\text { Torque } \\
\text { (\%) }\end{array}$ & $\begin{array}{c}\text { Shear Stress } \\
\qquad\left(\mathrm{N} / \mathrm{m}^{2}\right)\end{array}$ & $\begin{array}{c}\text { Shear Rate } \\
\left(\sec ^{-1}\right)\end{array}$ & $\begin{array}{c}\text { Viscosity } \\
\text { (mPa.s) }\end{array}$ & $\begin{array}{c}\text { Average } \\
\text { Viscosity } \\
\text { (mPa.s) }\end{array}$ \\
\hline 135 & 135.10 & 20.00 & 4.16 & 35.70 & 6.80 & 525.00 & \\
\hline 135 & 135.10 & 20.00 & 4.09 & 34.85 & 6.80 & 512.50 & 512.5 \\
\hline 135 & 135.10 & 20.00 & 3.98 & 34.00 & 6.80 & 500.00 & \\
\hline 140 & 140.10 & 20.00 & 2.76 & 23.80 & 6.80 & 350.00 & \\
\hline 140 & 140.20 & 20.00 & 2.73 & 22.95 & 6.80 & 337.50 & 341.7 \\
\hline 140 & 140.10 & 20.00 & 2.74 & 22.95 & 6.80 & 337.50 & \\
\hline 145 & 144.90 & 20.00 & 2.00 & 17.00 & 6.80 & 250.00 & \\
\hline 145 & 145.20 & 20.00 & 1.99 & 17.00 & 6.80 & 250.00 & 250.0 \\
\hline 145 & 145.10 & 20.00 & 1.99 & 17.00 & 6.80 & 250.00 & \\
\hline 150 & 150.00 & 20.00 & 1.45 & 12.75 & 6.80 & 187.50 & \\
\hline 150 & 150.10 & 20.00 & 1.44 & 11.90 & 6.80 & 175.00 & 179.2 \\
\hline 150 & 150.20 & 20.00 & 1.41 & 11.90 & 6.80 & 175.00 & \\
\hline 155 & 155.20 & 20.00 & 1.01 & 8.50 & 6.80 & 125.00 & \\
\hline 155 & 154.80 & 20.00 & 1.00 & 8.50 & 6.80 & 125.00 & 125.0 \\
\hline 155 & 154.80 & 20.00 & 0.98 & 8.50 & 6.80 & 125.00 & \\
\hline 160 & 160.00 & 20.00 & 0.69 & 5.95 & 6.80 & 87.50 & \\
\hline 160 & 159.90 & 20.00 & 0.69 & 5.95 & 6.80 & 87.50 & 87.50 \\
\hline 160 & 159.90 & 20.00 & 0.67 & 5.95 & 6.80 & 87.50 & \\
\hline 165 & 165.10 & 20.00 & 0.43 & 3.40 & 6.80 & 50.00 & \\
\hline 165 & 164.80 & 20.00 & 0.41 & 3.40 & 6.80 & 50.00 & 50.00 \\
\hline 165 & 164.80 & 20.00 & 0.42 & 3.40 & 6.80 & 50.00 & \\
\hline
\end{tabular}

Table 4.3 RV Temperature Sweep Test 18 Results 


\begin{tabular}{|c|c|c|c|c|c|c|c|c|}
\hline $\begin{array}{c}\text { Test } \\
\text { Number }\end{array}$ & $\begin{array}{c}\text { Test Temp. } \\
\left({ }^{\circ} \mathrm{C}\right)\end{array}$ & $\begin{array}{c}\text { Actual Temp. } \\
\left({ }^{\circ} \mathrm{C}\right)\end{array}$ & $\begin{array}{l}\text { Speed } \\
\text { (RPM) }\end{array}$ & $\begin{array}{c}\text { Torque } \\
\text { (\%) }\end{array}$ & $\begin{array}{l}\text { Shear } \\
\text { Stress } \\
\left(\mathrm{N} / \mathrm{m}^{2}\right)\end{array}$ & $\begin{array}{c}\text { Shear } \\
\text { Rate } \\
\left(\sec ^{-1}\right)\end{array}$ & $\begin{array}{c}\text { Viscosity } \\
\text { (mPa.s) }\end{array}$ & $\begin{array}{c}\text { Average } \\
\text { Viscosity } \\
\text { (mPa.s) }\end{array}$ \\
\hline \multirow[t]{3}{*}{19} & 135 & 135.00 & 20.00 & 4.01 & 34.00 & 6.80 & 500.00 & \\
\hline & 135 & 135.10 & 20.00 & 3.94 & 33.15 & 6.80 & 487.50 & 491.67 \\
\hline & 135 & 135.10 & 20.00 & 3.92 & 33.15 & 6.80 & 487.50 & \\
\hline \multirow[t]{3}{*}{20} & 135 & 135.10 & 20.00 & 4.06 & 34.85 & 6.80 & 512.50 & \\
\hline & 135 & 135.10 & 20.00 & 4.02 & 34.00 & 6.80 & 500.00 & 504.17 \\
\hline & 135 & 135.00 & 20.00 & 3.98 & 34.00 & 6.80 & 500.00 & \\
\hline \multirow[t]{3}{*}{21} & 140 & 140.10 & 20.00 & 2.83 & 23.80 & 6.80 & 350.00 & \\
\hline & 140 & 139.80 & 20.00 & 2.81 & 23.80 & 6.80 & 350.00 & 350.00 \\
\hline & 140 & 139.90 & 20.00 & 2.78 & 23.80 & 6.80 & 350.00 & \\
\hline \multirow[t]{3}{*}{22} & 140 & 139.90 & 20.00 & 2.71 & 22.95 & 6.80 & 337.50 & \\
\hline & 140 & 139.80 & 20.00 & 2.70 & 22.95 & 6.80 & 337.50 & 337.50 \\
\hline & 140 & 140.00 & 20.00 & 2.67 & 22.95 & 6.80 & 337.50 & \\
\hline \multirow[t]{3}{*}{23} & 145 & 145.00 & 20.00 & 2.03 & 17.00 & 6.80 & 250.00 & \\
\hline & 145 & 145.30 & 20.00 & 1.98 & 17.00 & 6.80 & 250.00 & 250.00 \\
\hline & 145 & 145.10 & 20.00 & 1.97 & 17.00 & 6.80 & 250.00 & \\
\hline \multirow[t]{3}{*}{24} & 145 & 145.10 & 20.00 & 2.10 & 17.85 & 6.80 & 262.50 & \\
\hline & 145 & 145.00 & 20.00 & 2.06 & 17.85 & 6.80 & 262.50 & 262.50 \\
\hline & 145 & 144.80 & 20.00 & 2.06 & 17.85 & 6.80 & 262.50 & \\
\hline \multirow[t]{3}{*}{25} & 150 & 149.80 & 20.00 & 1.95 & 17.00 & 6.80 & 250.00 & \\
\hline & 150 & 149.90 & 20.00 & 1.96 & 17.00 & 6.80 & 250.00 & 245.83 \\
\hline & 150 & 150.10 & 20.00 & 1.94 & 16.15 & 6.80 & 237.50 & \\
\hline \multirow[t]{3}{*}{26} & 150 & 150.30 & 20.00 & 1.45 & 12.75 & 6.80 & 187.50 & \\
\hline & 150 & 150.20 & 20.00 & 1.43 & 11.90 & 6.80 & 175.00 & 179.17 \\
\hline & 150 & 150.00 & 20.00 & 1.40 & 11.90 & 6.80 & 175.00 & \\
\hline \multirow[t]{3}{*}{27} & 155 & 155.20 & 20.00 & 1.63 & 13.60 & 6.80 & 200.00 & \\
\hline & 155 & 154.80 & 20.00 & 1.61 & 13.60 & 6.80 & 200.00 & 200.00 \\
\hline & 155 & 154.70 & 20.00 & 1.62 & 13.60 & 6.80 & 200.00 & \\
\hline \multirow[t]{3}{*}{28} & 155 & 154.90 & 20.00 & 1.76 & 15.30 & 6.80 & 225.00 & \\
\hline & 155 & 155.30 & 20.00 & 1.74 & 14.45 & 6.80 & 212.50 & 216.67 \\
\hline & 155 & 155.30 & 20.00 & 1.71 & 14.45 & 6.80 & 212.50 & \\
\hline \multirow[t]{3}{*}{29} & 160 & 160.10 & 20.00 & 1.37 & 11.90 & 6.80 & 175.00 & \\
\hline & 160 & 159.80 & 20.00 & 1.33 & 11.05 & 6.80 & 162.50 & 166.67 \\
\hline & 160 & 160.10 & 20.00 & 1.33 & 11.05 & 6.80 & 162.50 & \\
\hline \multirow[t]{3}{*}{30} & 160 & 160.00 & 20.00 & 0.57 & 5.10 & 6.80 & 75.00 & \\
\hline & 160 & 159.90 & 20.00 & 0.57 & 5.10 & 6.80 & 75.00 & 75.00 \\
\hline & 160 & 159.90 & 20.00 & 0.55 & 5.10 & 6.80 & 75.00 & \\
\hline \multirow[t]{3}{*}{31} & 165 & 165.00 & 20.00 & 0.27 & 2.55 & 6.80 & 37.50 & \\
\hline & 165 & 164.80 & 20.00 & 0.26 & 2.55 & 6.80 & 37.50 & 37.50 \\
\hline & 165 & 164.90 & 20.00 & 0.26 & 2.55 & 6.80 & 37.50 & \\
\hline \multirow[t]{3}{*}{32} & 165 & 165.10 & 20.00 & 0.42 & 3.40 & 6.80 & 50.00 & \\
\hline & 165 & 164.80 & 20.00 & 0.45 & 3.40 & 6.80 & 50.00 & 50.00 \\
\hline & 165 & 164.80 & 20.00 & 0.42 & 3.40 & 6.80 & 50.00 & \\
\hline
\end{tabular}

Table 4.4 RV Single Temperature Tests 19 through 32 Results 
The equivalent centimeter gram second (CGS) units are as follows:

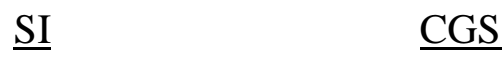

$\begin{array}{llll}\text { Viscosity } & 1 \mathrm{mPa} \cdot \mathrm{s} & = & 1 \mathrm{cP} \text { (centiPoise) } \\ \text { Shear Stress } & 1 \mathrm{~N} / \mathrm{m}^{2} & = & 10 \mathrm{dyne} / \mathrm{cm}^{2}\end{array}$

where: $1000 \mathrm{cP}=\left(\frac{10 \text { dyne } \cdot \mathrm{s}}{\mathrm{cm}^{2}}\right)=10 \mathrm{P}=1 \mathrm{~Pa} \cdot \mathrm{s}$

The SHRP binder specifications for pumpability require a maximum binder viscosity limit of $3 \mathrm{~Pa} \cdot \mathrm{s}$ when tested at $135^{\circ} \mathrm{C}$. As shown in Tables 4.2 through Table 4.4, each viscosity reading at $135^{\circ} \mathrm{C}$ is well below the $3 \mathrm{~Pa} \cdot \mathrm{s}$ limit, thus meeting the SHRP binder performance specifications.

There are no specification criteria for tests at temperatures greater than $135^{\circ} \mathrm{C}$. However, higher temperature tests are required to establish equiviscosity curves. As shown in Table 3.1, higher temperature testing was performed using both a single temperature per sample and temperature sweep methodologies.

Review of the data in Tables 4.2 through Table 4.4 demonstrates the sweep technique produces a decreasing viscosity with increasing temperature, as expected. However, some of the results for the tests conducted at single temperatures do not display expected behavior.

Test 25 , which was conducted at $150{ }^{\circ} \mathrm{C}$, appears to be erroneous since its average viscosity is significantly higher than the average viscosity of the duplicate Test 26 . The Test 25 average viscosity is also much greater than the average viscosity at $150{ }^{\circ} \mathrm{C}$ of duplicate temperature sweep Tests 17 and 18.

The average viscosities of duplicate Tests 27 and 28 are expectedly high as well. The average viscosities of Tests 27 and 28 , which were conducted at $155^{\circ} \mathrm{C}$, are significantly higher than those from duplicate temperature sweep Tests 17 and 18 at 
$155^{\circ} \mathrm{C}$. Furthermore, the viscosity of asphalt at $155{ }^{\circ} \mathrm{C}$ should be less than the viscosity at $150{ }^{\circ} \mathrm{C}$; however, the average viscosity results of duplicate Tests 27 and 28 are greater than the average viscosity of duplicate temperature sweep Tests 17 and 18 at $150{ }^{\circ} \mathrm{C}$. The average viscosity results of duplicate Tests 27 and 28 are also greater than the average viscosity of Test 26 , which was conducted at $150{ }^{\circ} \mathrm{C}$.

Test 29 appears to be invalid as well. Its average viscosity is much greater than the average viscosity of duplicate Test 30 . In addition, the average viscosity of Test 29 , is significantly greater than the average viscosity of duplicate temperature sweep Tests 17 and 18 conducted at $160{ }^{\circ} \mathrm{C}$.

A graphical display of the above observations is provided in Figure 4.1. The average viscosities of temperature sweep Test 17 are plotted and connected to create a viscosity-temperature curve. A viscosity-temperature curve for temperature sweep Test 18 is plotted on Figure 4.1 as well. As shown, both viscosity-temperature curves closely follow each other. The average viscosity of single temperature Tests 19 through 32 are plotted as data points only. Notice that Tests 19 through 24 and Test 31 and 32 produced average viscosities which are consistent with the average viscosities produced by temperature sweep Tests 17 and 18. Tests 25, 27, 28, and 29, however, produced unexpectedly high average viscosities, as previously discussed.

Tests 27, 29, and 28, were the first three RV tests conducted, as shown in Table 3.3. Therefore, it would be reasonable to assume that user error was a contributing factor to the erroreous results. However, Test 25 was the last test conducted. Therefore, user error with Test 25 is questionable.

A linear relationship between viscosity and temperature is desired for equiviscosity curves. To achieve this linear relationship, viscosity is plotted, by convention, on a log-log scale and temperature on a log scale. A log-log viscosity vs. log temperature plot of the Test 17 results is shown in Figure 4.2. The results of Test 17, however, do not exhibit the expected linear relationship. Thus, the results are suspect. 


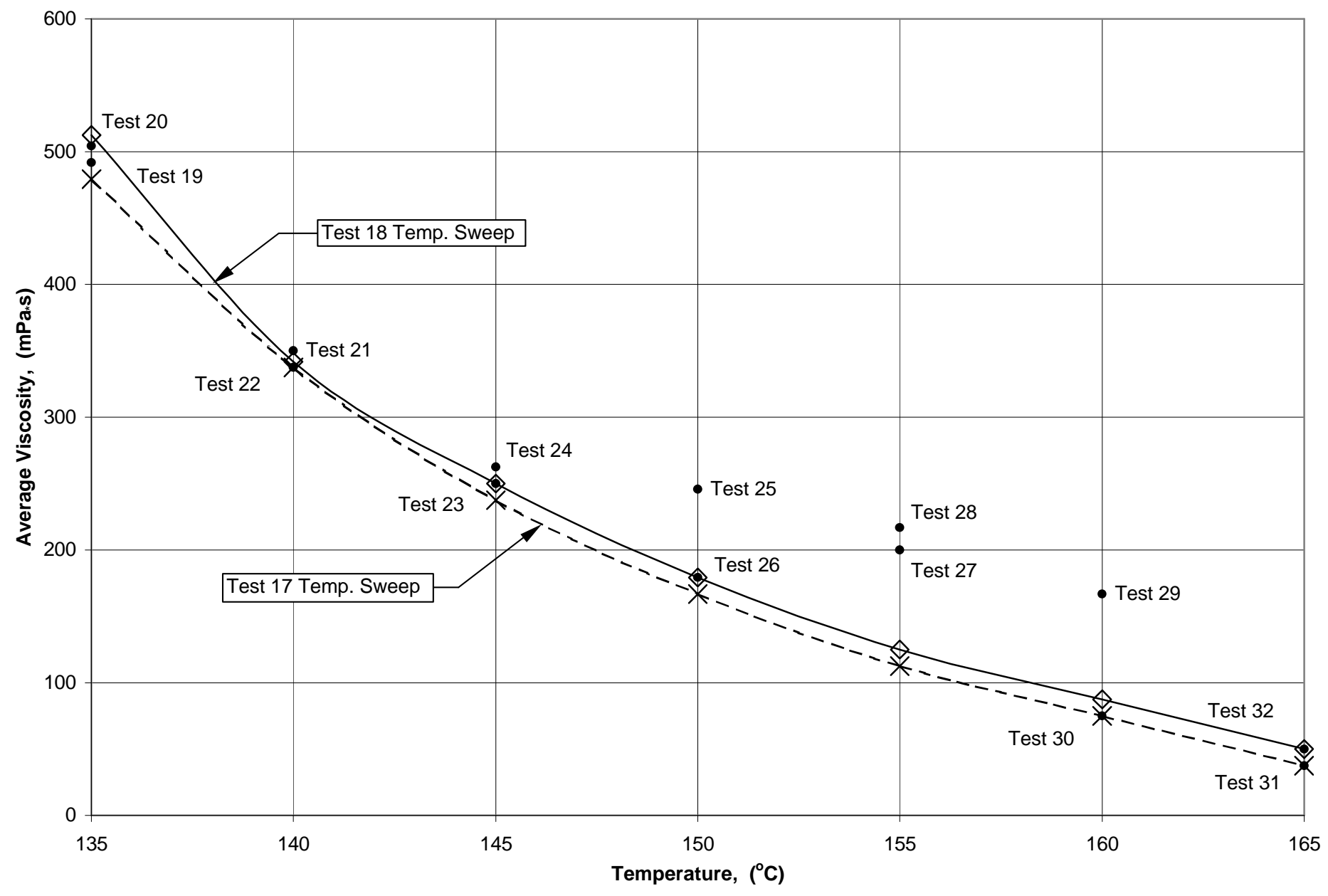

Figure 4.1 RV Viscosity vs. Temperature Plot 


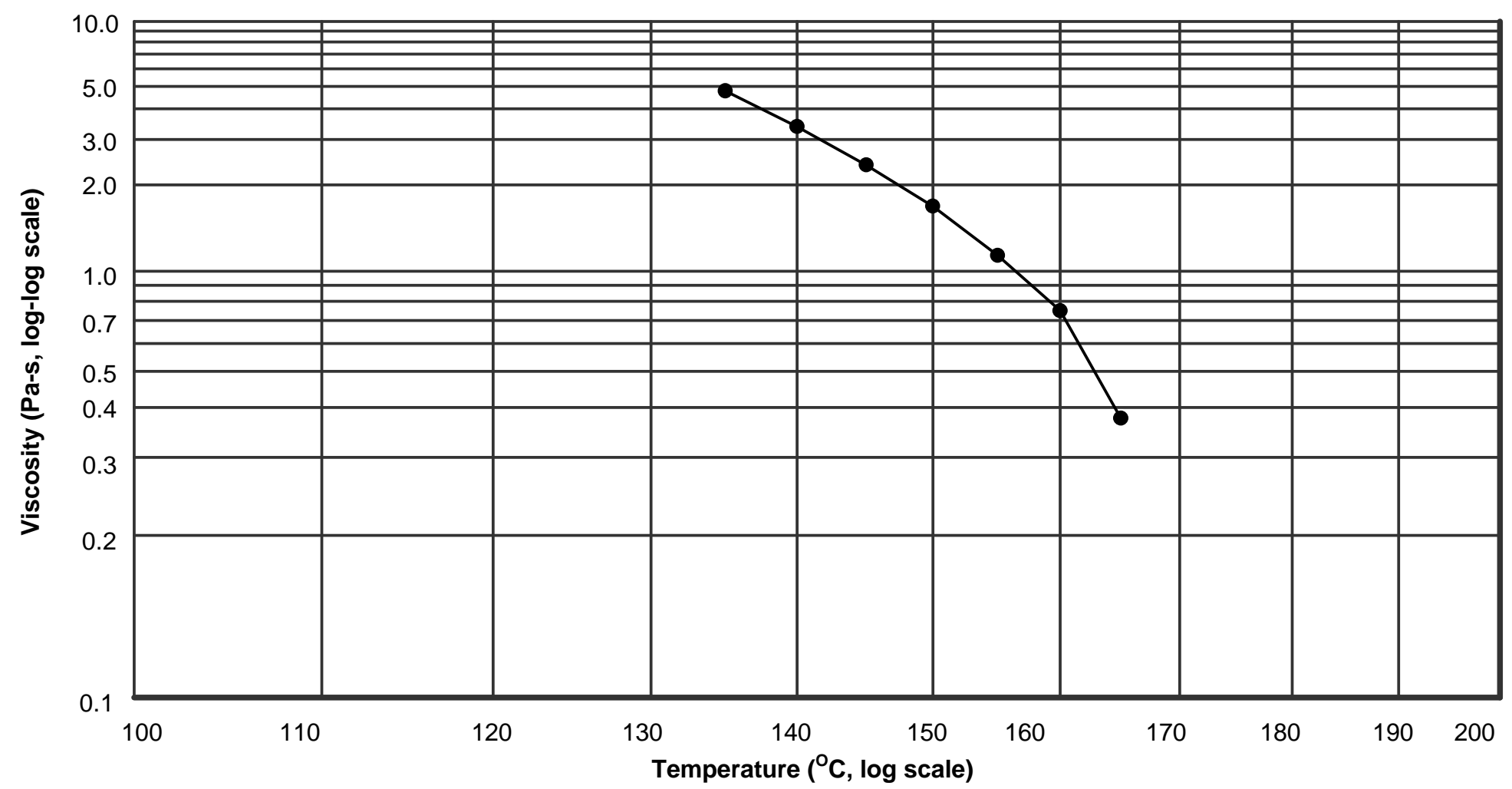

Figure 4.2 RV Test 17 Log-Log Viscosity vs. Log Temperature Plot 
The precision criteria of AASHTO TP48 requires that the difference in duplicate RV test results for a single operator in a single lab, expressed as a percent of their mean, shall not exceed 7.3 percent. A precision comparison of the average viscosity results at each test temperature for the duplicate RV temperature sweep Tests 17 and 18 is shown in Table 4.5. The average viscosity results from tests taken at and above $155^{\circ} \mathrm{C}$ failed the AASHTO precision criteria. As shown in Table 4.6, duplicate single temperature RV Tests 25 and 26, Tests 27 and 28, Tests 29 and 30, and Tests 31 and 32, of which test temperatures range from $150{ }^{\circ} \mathrm{C}$ and up, failed to meet the AASHTO precision criteria.

\begin{tabular}{|c|c|c|c|c|c|}
\hline $\begin{array}{c}\text { Test Temp. } \\
\left({ }^{\circ} \mathbf{C}\right)\end{array}$ & $\begin{array}{c}\text { Test 17 } \\
\text { Average } \\
\text { Viscosity } \\
\text { (mPa.s) }\end{array}$ & $\begin{array}{c}\text { Test 18 } \\
\text { Average } \\
\text { Viscosity } \\
\text { (mPa-s) }\end{array}$ & $\begin{array}{c}\text { Average } \\
\text { Viscosity } \\
\text { \% Difference }\end{array}$ & $\begin{array}{c}\text { Maximum } \\
\text { Allowable } \\
\text { \% Difference }\end{array}$ & Results \\
\hline 135 & 479.17 & 512.50 & 6.7 & 7.3 & PASSED \\
\hline 140 & 337.50 & 341.67 & 1.2 & 7.3 & PASSED \\
\hline 145 & 237.50 & 250.00 & 5.1 & 7.3 & PASSED \\
\hline 150 & 166.67 & 179.17 & 7.2 & 7.3 & PASSED \\
\hline 155 & 112.50 & 125.00 & 10.5 & 7.3 & FAlLED \\
\hline 160 & 75.00 & 87.50 & 15.4 & 7.3 & FAILED \\
\hline 165 & 37.50 & 50.00 & 28.6 & 7.3 & FAILED \\
\hline
\end{tabular}

${ }^{1}$ Per AASHTO TP48 Precision Criteria

Table 4.5 RV Temperature Sweep Tests Precision Results 


\begin{tabular}{|c|c|c|c|c|c|}
\hline Test Number & $\begin{array}{l}\text { Test Temp. } \\
\quad\left({ }^{\circ} \mathrm{C}\right)\end{array}$ & $\begin{array}{l}\text { Average } \\
\text { Viscosity } \\
\text { (mPa.s) }\end{array}$ & $\begin{array}{c}\text { Average } \\
\text { Viscosity } \\
\% \text { Difference }\end{array}$ & $\begin{array}{c}\text { Maximum } \\
\text { Allowable } \\
\% \text { Difference }\end{array}$ & Results \\
\hline $\begin{array}{l}19 \\
20\end{array}$ & $\begin{array}{l}135 \\
135\end{array}$ & $\begin{array}{l}491.67 \\
504.17\end{array}$ & 2.5 & 7.3 & PASSED \\
\hline $\begin{array}{l}21 \\
22\end{array}$ & $\begin{array}{l}140 \\
140\end{array}$ & $\begin{array}{l}350.00 \\
337.50\end{array}$ & 3.6 & 7.3 & PASSED \\
\hline $\begin{array}{l}23 \\
24\end{array}$ & $\begin{array}{l}145 \\
145\end{array}$ & $\begin{array}{l}250.00 \\
262.50\end{array}$ & 4.9 & 7.3 & PASSED \\
\hline $\begin{array}{l}25 \\
26\end{array}$ & $\begin{array}{l}150 \\
150\end{array}$ & $\begin{array}{l}245.83 \\
179.17\end{array}$ & 31.4 & 7.3 & FAILED \\
\hline $\begin{array}{l}27 \\
28\end{array}$ & $\begin{array}{l}155 \\
155\end{array}$ & $\begin{array}{l}200.00 \\
216.67\end{array}$ & 8.0 & 7.3 & FAILED \\
\hline $\begin{array}{l}29 \\
30\end{array}$ & $\begin{array}{l}160 \\
160\end{array}$ & $\begin{array}{l}166.67 \\
75.00\end{array}$ & 75.9 & 7.3 & FAILED \\
\hline $\begin{array}{l}31 \\
32\end{array}$ & $\begin{array}{l}165 \\
165\end{array}$ & $\begin{array}{l}37.50 \\
50.00\end{array}$ & 28.6 & 7.3 & FAILED \\
\hline
\end{tabular}

${ }^{1}$ Per AASHTO TP48 Precision Criteria

Table 4.6 RV Single Temperature Tests Precision Results

The observation that the average viscosity results failed the AASHTO precision criteria from test temperatures greater than $145^{\circ} \mathrm{C}$ and $150{ }^{\circ} \mathrm{C}$, for the individual tests and temperature sweep tests, respectively, raised questions regarding the precision of the Brookfield DV-III rotational viscometer. Therefore, the Brookfield Operating Instructions manual was reviewed to determine the manner in which the DV-III determines viscosity. The Brookfield DV-III viscometer determines the viscosity by first measuring the percent torque required to rotate the spindle at a constant speed. The torque is applied through a calibrated spring. The degree to which the spring is wound is used to calculate the applied torque. The percent torque value is rounded to one tenth of a percent and used in Equation 4-1 to calculate the viscosity. 


$$
\text { Viscosity }=\left(\frac{10,000}{\mathrm{RPM}} * \mathrm{TK} * \mathrm{SMC}\right)(\% \text { Torque })
$$

where: $\mathrm{RPM}=$ Spindle speed

$\mathrm{TK}=$ Model spring constant

$\mathrm{SMC}=$ Spindle multiplier constant

$\%$ Torque $=$ Percent torque expressed in decimal form

The spindle multiplier constant, SMC, of Equation 4-1 is based on the spindle type. The model spring constant, TK, of Equation 4-1 is based on the calibrated spring of the unit. There are four different model series of the Brookfield DV-III: LV, RV, HA, and HB model series. Each model series uses a different calibrated spring. An RV model series Brookfield DV-III, RVDV-III, was used during this research.

The product of $\left(\frac{10,000}{\mathrm{RPM}} * \mathrm{TK} * \mathrm{SMC}\right)$ in Equation $4-1$ is referred to as the fullscale viscosity range. The full-scale viscosity range for a RVDV-III equipped with a SC4-27 spindle and a 20-RPM operating speed is $12,500 \mathrm{mPa} \cdot \mathrm{s}$.

The shear rate is calculated as:

Shear Rate $=\mathrm{RPM} * \mathrm{SRC}$

where: SRC $=$ Spindle shear rate constant

Shear stress is calculated as:

Shear Stress $=100(\mathrm{TK} * \mathrm{SMC} * \mathrm{SRC} * \%$ Torque $)$

where: \% Torque is expressed in decimal form. 
The Brookfield DV-III viscometer determines the percent torque and then uses that value, rounded to the nearest tenth of a percent, to calculate the viscosity with Equation 4-1. Based on Equation 4-1 and the measuring capabilities of the Brookfield DV-III, a tenth of a percent change in torque yields a $12.5 \mathrm{mPa} \cdot \mathrm{s}$ change in viscosity when using an RV series viscometer with an SC4-27 spindle at 20 RPM. Thus, the smallest change in torque, a tenth of a percent, will result in $12.5 \mathrm{mPa} \cdot \mathrm{s}$ incremental changes in viscosity. AASHTO TP48 requires that the difference in duplicate test results for a single operator, expressed, as a percent of their mean, shall not exceed 7.3 percent. Since viscosity changes in increments of $12.5 \mathrm{mPa} \cdot \mathrm{s}$ and the maximum difference between test results shall not exceed $7.3 \%$, the minimum viscosity difference between two duplicate tests can be approximated as follows:

$$
\begin{aligned}
\text { Max. Allowable } \% \text { Difference } & =\left(\frac{\text { Viscosity Difference }}{\text { Average Viscosity }}\right) \\
7.3 \% & =\left(\frac{12.5 \mathrm{mPa} \cdot \mathrm{s}}{\text { Average } \mathrm{Viscosity}}\right)
\end{aligned}
$$

It follows from Equation 4-4 that duplicate tests, conducted with the Brookfield RVDV-III using a SC4-27 spindle at a 20-RPM operating speed, that produce average viscosities of less than $171 \mathrm{mPa} \cdot \mathrm{s}$ will fail the precision and bias criteria of AASHTO TP48. As shown in Tables 4.5 and 4.6, the duplicate tests on the neat PG 70-22 binder at temperatures above $150{ }^{\circ} \mathrm{C}$ yielded average viscosities less than $171 \mathrm{mPa} \cdot \mathrm{s}$. As a result, those tests failed the precision and bias criteria of AASHTO TP48.

Tests yielding viscosities lower than $171 \mathrm{mPa} \cdot \mathrm{s}$, which meet the precision and bias requirement of AASHTO TP48, can be conducted using the SHRP recommended SC4-27 spindle by reducing the full-scale viscosity range indicated by Equation 4-1. Reducing the full-scale viscosity range results in a smaller incremental change in viscosity per each tenth of a percent change in torque, which, in turn, yields a lower 
average viscosity that can be tested while meeting the precision and bias criteria of AASHTO TP48, as per Equation 4-4.

The full-scale viscosity range can be reduced via two different means. Increasing the spindle speed is one method for reducing the full-scale viscosity range. For example, by increasing the spindle speed to 30 RPM, the full-scale viscosity range of an RV series viscometer with a SC4-27 spindle is reduced to 8,333. Thus, a tenth of a percent change in torque will result in an 8.333 incremental change in viscosity. The minimum average viscosity that would meet the AASHTO precision and bias criteria for two duplicate test results using a SC4-27 spindle at $30 \mathrm{RPM}$ would be approximately $114 \mathrm{mPa} \cdot \mathrm{s}$, which in turn would allow tests to be conducted at higher temperatures.

Different spindles can also be used to reduce the full-scale viscosity range. An RV series viscometer with SC4-21 spindle operating at 20 RPM, for example, has a fullscale viscosity range of 2,500. The viscosity will change by $2.5 \mathrm{mPa} \cdot \mathrm{s}$ with each tenth of a percent change in torque. As a result, the minimum average viscosity, which would meet AASHTO precision and bias criteria, between two duplicate test results for a SC421 spindle at $20 \mathrm{RPM}$ would be approximately $68.5 \mathrm{mPa} \cdot \mathrm{s}$.

A different model series DV-III viscometer must be used if the required full-scale viscosity range cannot be achieved by changing the spindle type and/or operating speed. The viscometer must be selected with the appropriate spring constant to work in conjunction with the spindle type and operating speed combination to achieve the desired full-scale viscosity range.

As a result of the viscosity measuring method and capabilities of the Brookfield DV-III, operators must be aware of the minimum viscosity limits, which in turn limit the maximum test temperatures, in order to achieve the precision and bias criteria of AASHTO TP48. These limits are dependant upon the asphalt binder grade, viscometer spring constant, spindle type, and operating speed. 
In addition to the DV-III viscosity measuring method and capabilities observed during the review of the Brookfield Operating Instructions manual, it was also noted that the range of viscosity measurements that should be taken from the Brookfield DV-III viscometer extends from a minimum viscosity of $10 \%$ of the full-scale range to a maximum viscosity of $100 \%$ of the full-scale range. The accuracy of tests producing viscosities outside of the $10 \%$ to $100 \%$ full-scale range cannot be guaranteed. Thus, the minimum viscosity that can be reliably measured when using an RVDV-III with a SC427 spindle at $20 \mathrm{RPM}$ is $1250 \mathrm{mPa} \cdot \mathrm{s}$. The asphalt viscosities measured during the testing at $135{ }^{\circ} \mathrm{C}$ ranged from $475 \mathrm{mPa} \cdot \mathrm{s}$ to $525 \mathrm{mPa} \cdot \mathrm{s}$. The viscosities yielded by these tests are well below the $10 \%$ of full-scale range minimum allowable viscosity criterion of the Brookfield Operating Instructions manual. Thus, the accuracy of all the rotational tests is suspect.

An asphalt supplier was contacted to determine typical viscosity ranges of PG 70-22 asphalt and with what type of spindle and speed their tests were conducted. Typical viscosities of $\mathrm{PG} 70-22$ at $135{ }^{\circ} \mathrm{C}$ range from $555 \mathrm{mPa} \cdot \mathrm{s}$ to $600 \mathrm{mPa} \cdot \mathrm{s}$, as per the supplier's quality control data. The supplier uses a Brookfield RVDV-II viscometer; the typical viscosities provided by the supplier were obtained using a SC4-21 spindle and a 20-RPM operating speed. A Brookfield technician was contacted to determine if the Brookfield DV-II models used by the supplier have the same measuring and precision capabilities as the DV-III model used for this research. The Brookfield technician verified that the DV-II and DV-III viscometer models share the same measuring and precision capabilities. In addition, the spring constant for the RV model series is the same for both the DV-II and DV-III models (Thibodeau, 2003).

The allowable viscosity range using a SC4-21 spindle operating at 20 RPM is 250 to $2,500 \mathrm{mPa} \cdot \mathrm{s}$ (i.e. $10 \%$ to $100 \%$ of the 2,500 full-scale viscosity range); therefore, the viscosities at $135{ }^{\circ} \mathrm{C}$ obtained by the supplier are within the allowable limits of the Brookfield RVDV-II. Although the results obtained during this research are close to the typical viscosity range provided by the asphalt supplier, they are out of the accuracy range of the RVDV-III with a SC4-27 spindle at the 20-RPM operating speed. Therefore, 
using an SC4-27 spindle at 20 RPM with RV model series Brookfield viscometers should be avoided when testing PG binders at temperatures that produce viscosities less than $1250 \mathrm{mPa} \cdot \mathrm{s}$. When using an RVDV-II or RVDV-III to perform tests at $135{ }^{\circ} \mathrm{C}$ to verify the pumping ability of PG 70-22 asphalt binder, spindle speeds of at least 45 RPM would be needed in combination with the SC4-27 spindle in order to achieve viscosities above the $10 \%$ full-scale range limit, as per the manufacturer's criterion.

Based upon the above information, the combination of a SC4-27 spindle at 20 RPM is not applicable to all tests. The selection of spindle type and speed is dependant upon the viscometer spring constant, asphalt grade and test temperatures. Unfortunately, ambiguity on this detail exists in asphalt industry protocol, as discussed in Chapter 3. A SC4-27 spindle is recommended in the SHRP literature (Petersen, et al., 1994b). A 20RPM operating speed is also recommended by the SHRP researchers in a different volume of the final report (Anderson, et al., 1994). No recommendation was found that united the spindle selection and operating speed. The SHRP researchers used a Brookfield DV-II viscometer for their testing; however, the viscometer series (LV, RV, HA, or HB) was not documented (Anderson, et al., 1994). A plot of viscositytemperature curves generated using capillary and Brookfield DV-II viscometers during the SHRP research is shown in Figure 4.3. The Brookfield tests used to construct the viscosity-temperature curve of Figure 4.3 were conducted using an SC4-27 spindle at 20 RPM. The test results are less than the minimum viscosity that can be reliably measured using that spindle-operating speed combination with an RVDV-II viscometer, as shown graphically in Figure 4.3 with the addition of the reliability limit. If the SHRP researchers used an RVDV-II viscometer, then SHRP test results such as those in Figure 4.3 are suspect.

The use of incorrect spindle and operating speed combinations can lead to erroneous equiviscosity curves. A linear relationship is expected creating log-log viscosity vs. log temperature plots. This linear relationship allows for the development of equiviscosity curves by conducting tests at only two temperatures, as per both the SHRP and Asphalt Institute methods. When developing equiviscosity curves at only two 
temperatures; however, this linear relationship is assumed. For example, consider Figure 4.4, the log-log viscosity vs. log temperature plot of the temperature sweep Test 17 data, which is considered suspect.since the tests were conducted outside the measuring capabilities of the Brookfield RVDV-III due to the spindle and operating speed combinations used. If an operator was unaware of the importance of proper spindle-operating speed selection and only plotted the Test 17 data points at $135{ }^{\circ} \mathrm{C}$ and $165^{\circ} \mathrm{C}$, as per the Asphalt Institute recommendations, the result would be an equiviscosity curve that does not reflect the actual test data curve. In this case, the assumption that the a linear relationship exists would be incorrect, thus leading to an erroneous equiviscosity curve. Therefore, it is critical that the tests are conducted within the measuring capabilities of the rotational viscometer to produce accurate equiviscosity curves when testing at only two temperatures. Furthermore, testing at three or more temperatures would also help verify the accuracy of the equiviscosity curves.

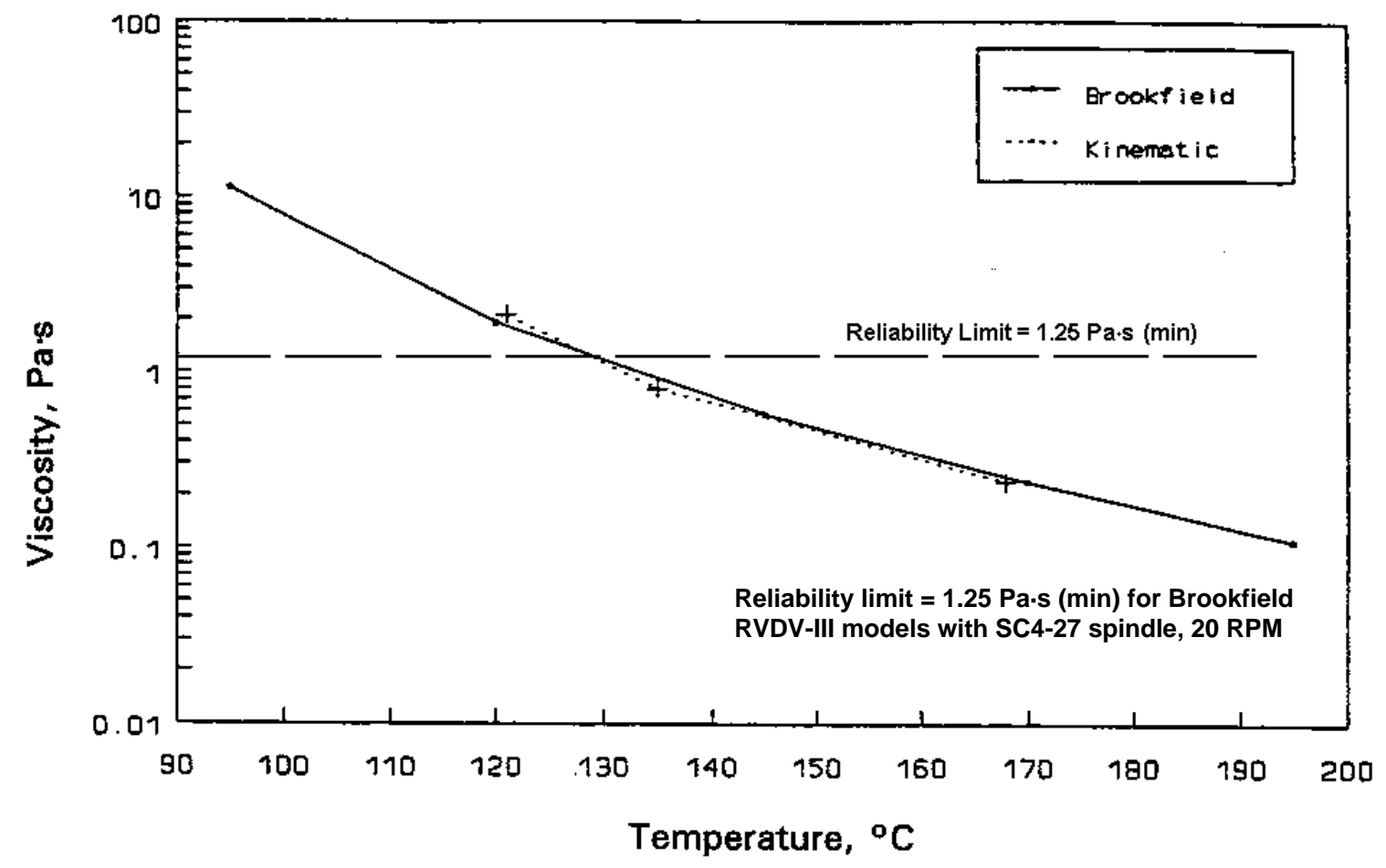

Figure 4.3 Viscosity Measured by SHRP Researchers with Capillary and Brookfield Viscometers, SC4-27 Spindle at 20 RPM 


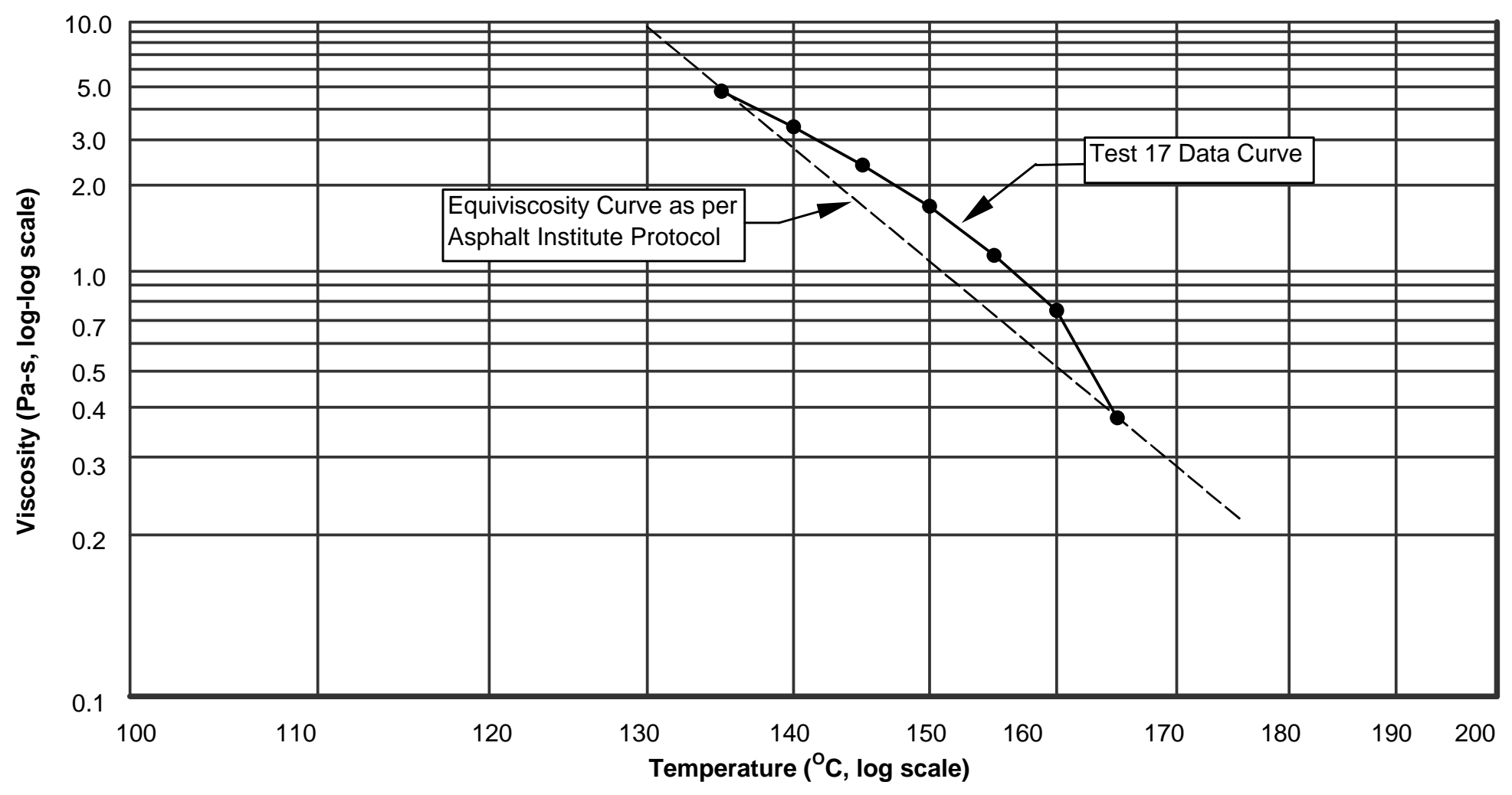

Figure 4.4 RV Temperature Sweep Test 17 Equiviscosity Curve 
The concern that other research facilities and the asphalt industry could misinterpret the existing testing protocol and other reference sources (i.e. AASHTO standard specifications, Asphalt Institute publications, etc.) was justified following the inquiry with the aforementioned asphalt supplier for their typical viscosities of PG 70-22 asphalt at $165{ }^{\circ} \mathrm{C}$. The range of viscosities for $\mathrm{PG} 70-22$ asphalt at $165{ }^{\circ} \mathrm{C}$ varied from $150 \mathrm{mPa} \cdot \mathrm{s}$ to $160 \mathrm{mPa} \cdot \mathrm{s}$, as per the supplier's quality control data. These results were from tests conducted with an RVDV-II using a SC4-21 spindle operating at 20 RPM. As described above, the minimum viscosity that can be reliably measured with an RVDV-II viscometer using a SC4-21 spindle at $20 \mathrm{RPM}$ is $250 \mathrm{mPa} \cdot \mathrm{s}$. Thus, the viscosity range cited by the asphalt supplier is suspect since it is below the allowable limits of the Brookfield RVDV-II viscometer.

\section{Equipment Evaluation}

The rotational viscometer test required minimal user input. The unit was simple to calibrate and operate. Once the basic sample handling and preparation steps where completed, the rotational viscometer software controlled the remainder of the test. Therefore, user proficiency was quickly developed.

Problems with test temperature recording were experienced early in the trial testing period. The test temperature recorded on the Thermosel unit did not match the temperature displayed on the computer monitor, as determined by the Brookfield software. The AASHTO specifications require that the temperature of the test specimen be maintained to within $\pm 0.1{ }^{\circ} \mathrm{C}$ of the test temperature. Temperatures differences between the Thermosel and the Brookfield software, however, were observed to be greater than the $\pm 0.1{ }^{\circ} \mathrm{C}$ allowable difference. A Brookfield technician was contacted to obtain guidance on the observed temperature discrepancy. The technician advised that the computer display was correct; therefore, the Brookfield software temperature display was used as the standard calibration and testing throughout the testing program.

As previously described, $1250 \mathrm{mPa} \cdot \mathrm{s}$ is the minimum viscosity that can be reliably measured by the Brookfield RVDV-III when using an SC4-27 spindle at 20 
RPM. The viscosity results obtained from the testing program; however, were much lower than $1250 \mathrm{mPa} \cdot \mathrm{s}$, ranging from $525 \mathrm{mPa} \cdot \mathrm{s}$ to $37.50 \mathrm{mPa} \cdot \mathrm{s}$, as shown in Tables 4.2 through Table 4.4. Therefore, the erroneous results of Tests 25, 27, 28, and 29 are expected since their viscosities could not be reliably measured. Furthermore, none of the tests results can be considered reliable.

\section{Test Protocol and Specifications}

The performance grading specification for pumpability of AASHTO MP1 requires that the viscosity at $135{ }^{\circ} \mathrm{C}$ must not exceed $3 \mathrm{~Pa} \cdot \mathrm{s}$. It is noteworthy that a material specification for viscosity-grading system exists for the viscosity at $135{ }^{\circ} \mathrm{C}$ as well. The viscosity-grading system criterion, which is provided in ASTM 3381, established minimum viscosities ranging from $0.08 \mathrm{~Pa} \cdot \mathrm{s}$ to $0.3 \mathrm{~Pa} \cdot \mathrm{s}$ at $135{ }^{\circ} \mathrm{C}$, depending on the asphalt cement grade. Presumably, this specification was established to control asphalt drain-down during storage and transportation. A minimum specification is not provided in the PG system; it only requires a maximum viscosity for pumpability. No documentation was found in the literature to justify this fundamental change in specifications.

Equiviscous temperature ranges have been used for the past 20 years for determining laboratory mixing and compaction temperatures (Asphalt Institute, 2001). The capillary tube viscometer was used with the Marshall mix design methodology for determining equiviscous temperatures. The capillary tube viscometers tests were conducted at $60{ }^{\circ} \mathrm{C}$ and $135{ }^{\circ} \mathrm{C}$, which, in turn, required extrapolation to estimate mixing and compaction temperatures that can exceed $150{ }^{\circ} \mathrm{C}$. The AASHTO TP48 test procedure for rotational viscometer testing recognizes the need for determining asphalt equiviscous temperatures for mixing and compaction. However, the AASHTO test method does not provide test temperature specifications for creating viscositytemperature profiles. The Asphalt Institute (2001) recommends determining asphalt viscosities at $135{ }^{\circ} \mathrm{C}$ and $165{ }^{\circ} \mathrm{C}$ test temperatures, which allows for the determination of equiviscous temperatures via interpolation. The Asphalt Institute recommendation is appealing since it avoids extrapolation issues. However, the very low viscosity of asphalt 
at $165{ }^{\circ} \mathrm{C}$ requires appropriate selection of the rotational viscometer spindle and test speed.

\subsection{Dynamic Shear Rheometer}

\section{Testing Results Analysis}

A summary of the dynamic shear rheometer test results are shown in

Table 4.7. The results of the test are checked against SHRP performance specifications $\mathrm{G}^{*} / \sin \delta$ and $\mathrm{G}^{*} \sin \delta$, which were discussed in Chapter 2 . Test 6 was the only test that failed to meet the SHRP specifications; it did not achieve the minimum $G^{*} / \sin \delta$ requirement of $2.2 \mathrm{kPa}$ for RTFO-aged binder. A second test, Test $6 \mathrm{~b}$, was conducted from a reserve tin in Sample Set 3. It failed to achieve the minimum $\mathrm{G}^{*} / \sin \delta$ requirement as well. Test 6 was the $7^{\text {th }}$ of 16 DSR tests conducted, as shown in Table 3.3. As shown in Table 3.2, the sample set from which Test 6 was conducted, Sample Set 3, was the last sample set to undergo RTFO conditioning. Thus, user error with the RTFO as a contributing factor to the failed test result is minimal; however, user error with the DSR is possible.

A comparative precision analysis between the duplicate test results is provided in Table 4.8. The single-operator precision criteria of AASHTO TP5 requires that the difference in duplicate DSR test results for a single operator in the same lab, expressed as a percent of their mean, shall not exceed 9.5 percent when testing neat asphalt, 11.0 percent when testing RTFO-aged asphalt, and 22.4 percent when testing PAV-aged asphalt. A comparison between Tests 5 and 6 was not applicable since Test 6 was not valid. Duplicate Tests 35 and 36 were the only tests to fail the precision requirements of AASHTO TP5. Test 36 is an outlier when compared to the results of Tests 33, 34, and 35 ; of which all were conducted on neat asphalt. Test 36 was the $5^{\text {th }}$ DSR test conducted; therefore, user error is a possible factor in the unexpected results. 


\begin{tabular}{|c|c|c|c|c|c|c|c|c|}
\hline \multicolumn{7}{|c|}{ HIGH TEMPERATURE RANGE TEST RESULTS: RTFO-AGED BINDER } \\
\hline $\begin{array}{c}\text { Test } \\
\text { Number }\end{array}$ & $\begin{array}{c}\text { Frequency } \\
(\text { Rad/sec) }\end{array}$ & $\begin{array}{c}\text { Final } \\
\text { Temp. } \\
\left({ }^{\circ} \mathbf{C}\right)\end{array}$ & $\begin{array}{c}\text { Strain } \\
\text { Amplitude } \\
\text { (Percent) }\end{array}$ & $\begin{array}{c}\text { Modulus, G* } \\
(\mathbf{k P a})\end{array}$ & $\begin{array}{c}\text { Phase } \\
\text { Angle, } \delta \\
(\text { Degrees) }\end{array}$ & $\begin{array}{c}\text { Minimum } / \mathbf{s i n} \delta \\
\mathbf{( k P a )}\end{array}$ & $\begin{array}{c}\text { Allowable } \\
\mathbf{G}^{*} / \mathbf{s i n} \delta \\
(\mathbf{k P a})\end{array}$ & $\begin{array}{c}\text { Test } \\
\text { Status }\end{array}$ \\
\hline 1 & 10.08 & 70 & 10.01 & 2.546 & 83.2 & 2.564 & 2.2 & Passed \\
\hline 2 & 10.08 & 70 & 9.89 & 2.564 & 83.6 & 2.580 & 2.2 & Passed \\
\hline 3 & 10.08 & 70 & 10.01 & 2.636 & 83.1 & 2.656 & 2.2 & Passed \\
\hline 4 & 10.08 & 70 & 10.02 & 2.714 & 82.7 & 2.737 & 2.2 & Passed \\
\hline 5 & 10.08 & 70 & 10.29 & 2.463 & 83.1 & 2.481 & 2.2 & Passed \\
\hline 6 & 10.08 & 70 & 9.93 & 2.120 & 83.4 & $\mathbf{2 . 1 3 4}$ & $\mathbf{2 . 2}$ & Failed \\
\hline $6 \mathrm{~b}$ & 10.08 & 70 & 9.98 & 2.019 & 84.3 & $\mathbf{2 . 0 2 9}$ & $\mathbf{2 . 2}$ & Failed \\
\hline 7 & 10.08 & 70 & 10.06 & 2.410 & 83.3 & 2.427 & 2.2 & Passed \\
\hline 8 & 10.08 & 70 & 10.06 & 2.548 & 83.0 & 2.567 & 2.2 & Passed \\
\hline
\end{tabular}

\begin{tabular}{|c|c|c|c|c|c|c|c|c|}
\hline \multicolumn{7}{|c|}{ INTERMEDIATE TEMPERATURE RANGE TEST RESULTS: PAV-AGED BINDER } \\
\hline $\begin{array}{c}\text { Test } \\
\text { Number }\end{array}$ & $\begin{array}{c}\text { Frequency } \\
(\mathbf{R a d} / \mathbf{s e c})\end{array}$ & $\begin{array}{c}\text { Final } \\
\text { Temp. } \\
\left({ }^{\circ} \mathbf{C}\right)\end{array}$ & $\begin{array}{c}\text { Strain } \\
\text { Amplitude } \\
\text { (Percent) }\end{array}$ & $\begin{array}{c}\text { Modulus, G** } \\
\mathbf{( k P a )}\end{array}$ & $\begin{array}{c}\text { Phase } \\
\text { Angle, } \delta \\
(\text { Degrees) }\end{array}$ & $\begin{array}{c}\text { G*sin } \delta \\
\mathbf{( k P a )}\end{array}$ & $\begin{array}{c}\text { Maximum } \\
\text { Allowable } \\
\mathbf{G}^{*} \mathbf{s i n} \delta \\
\mathbf{( k P a )}\end{array}$ & $\begin{array}{c}\text { Test } \\
\text { Status }\end{array}$ \\
\hline 9 & 10.08 & 28 & 1.03 & 3879 & 50.6 & 2999 & 5000 & Passed \\
\hline 10 & 10.08 & 28 & 1.01 & 3722 & 50.5 & 2873 & 5000 & Passed \\
\hline 13 & 10.08 & 28 & 1.01 & 3641 & 50.4 & 2804 & 5000 & Passed \\
\hline 14 & 10.08 & 28 & 1.00 & 3226 & 50.4 & 2486 & 5000 & Passed \\
\hline
\end{tabular}

\begin{tabular}{|c|c|c|c|c|c|c|c|c|}
\hline \multicolumn{8}{|c|}{ HIGH TEMPERATURE RANGE TEST RESULTS: UNAGED BINDER } \\
\hline $\begin{array}{c}\text { Test } \\
\text { Number }\end{array}$ & $\begin{array}{c}\text { Frequency } \\
(\text { Rad/sec) }\end{array}$ & $\begin{array}{c}\text { Final } \\
\text { Temp. } \\
\left({ }^{\circ} \mathbf{C}\right)\end{array}$ & $\begin{array}{c}\text { Strain } \\
\text { Amplitude } \\
\text { (Percent) }\end{array}$ & $\begin{array}{c}\text { Modulus, G** } \\
\mathbf{( k P a )}\end{array}$ & $\begin{array}{c}\text { Phase } \\
\text { Angle, } \delta \\
(\text { Degrees) }\end{array}$ & $\begin{array}{c}\text { G*/sin } \delta \\
\mathbf{( k P a )}\end{array}$ & $\begin{array}{c}\text { Minimum } \\
\text { Allowable } \\
\mathbf{G} / \mathbf{s i n} \delta \\
(\mathbf{k P a})\end{array}$ & $\begin{array}{c}\text { Test } \\
\text { Status }\end{array}$ \\
\hline 33 & 10.08 & 70 & 12.06 & 1.207 & 84.4 & 1.213 & 1.0 & Passed \\
\hline 34 & 10.08 & 70 & 12.07 & 1.200 & 84.6 & 1.205 & 1.0 & Passed \\
\hline 35 & 10.08 & 70 & 11.99 & 1.224 & 85.1 & 1.229 & 1.0 & Passed \\
\hline 36 & 10.08 & 70 & 11.83 & 1.074 & 85.1 & 1.078 & 1.0 & Passed \\
\hline
\end{tabular}

Table 4.7 DSR Test Results 


\begin{tabular}{|c|c|c|c|c|}
\hline \multicolumn{5}{|c|}{$\begin{array}{l}\text { HIGH TEMPERATURE RANGE TEST RESULTS ANALYSIS: } \\
\text { RTFO-AGED BINDER }\end{array}$} \\
\hline $\begin{array}{c}\text { Test } \\
\text { Number }\end{array}$ & $\begin{array}{c}\mathrm{G}^{*} / \sin \delta \\
(\mathrm{kPa})\end{array}$ & $\begin{array}{c}\text { Average } \\
\mathbf{G}^{\star} / \sin \delta \\
\% \text { Difference }\end{array}$ & $\begin{array}{c}\text { Maximum } \\
\text { Allowable } \\
\% \text { Difference }\end{array}$ & Results \\
\hline 1 & $\begin{array}{l}2.564 \\
2.580 \\
\end{array}$ & 0.62 & 11 & Passed \\
\hline $\begin{array}{l}3 \\
4\end{array}$ & $\begin{array}{l}2.656 \\
2.737 \\
\end{array}$ & 3.00 & 11 & Passed \\
\hline 5 & $\begin{array}{r}2.481 \\
\text { *Failed the }\end{array}$ & minimum $\mathbf{G}^{*} / \mathrm{s}$ & in $\delta$ criteria* & N/A \\
\hline $\begin{array}{l}7 \\
8\end{array}$ & $\begin{array}{l}2.427 \\
2.567\end{array}$ & 5.61 & 11 & Passed \\
\hline
\end{tabular}

\begin{tabular}{|c|c|c|c|c|}
\hline \multicolumn{5}{|c|}{$\begin{array}{l}\text { INTERMEDIATE TEMPERATURE RANGE TEST RESULTS } \\
\text { ANALYSIS: PAV-AGED BINDER }\end{array}$} \\
\hline $\begin{array}{c}\text { Test } \\
\text { Number }\end{array}$ & $\begin{array}{l}\mathbf{G}^{*} \sin \delta \\
(\mathrm{kPa})\end{array}$ & $\begin{array}{c}\text { Average } \\
\mathbf{G}^{*} \sin \delta \\
\% \text { Difference }\end{array}$ & $\begin{array}{c}\text { Maximum } \\
\text { Allowable } \\
\% \text { Difference }\end{array}$ & Results \\
\hline $\begin{array}{r}9 \\
10 \\
\end{array}$ & $\begin{array}{l}2999 \\
2873 \\
\end{array}$ & 4.29 & 22.4 & Passed \\
\hline $\begin{array}{l}13 \\
14 \\
\end{array}$ & $\begin{array}{l}2804 \\
2486 \\
\end{array}$ & 12.02 & 22.4 & Passed \\
\hline
\end{tabular}

${ }^{1}$ Per AASHTO TP5 Precision Criteria

\begin{tabular}{|c|c|c|c|c|}
\hline \multicolumn{5}{|c|}{ HIGH TEMPERATURE RANGE TEST RESULTS ANALYSIS: } \\
UNAGED BINDER \\
\hline $\begin{array}{c}\text { Test } \\
\text { Number }\end{array}$ & $\begin{array}{c}\text { G*/sin } \delta \\
(\mathbf{k P a})\end{array}$ & $\begin{array}{c}\text { Average } \\
\text { G*/sin } \delta \\
\% \text { Difference }\end{array}$ & $\begin{array}{c}\text { Maximum } \\
\text { Allowable } \\
\% \text { Difference }\end{array}$ & Results \\
\hline 33 & 1.213 & 0.66 & 9.5 & Passed \\
34 & 1.205 & 13.09 & 9.5 & Failed \\
\hline 35 & 1.229 & 1.078 & &
\end{tabular}

${ }^{1}$ Per AASHTO TP5 Precision Criteria

Table 4.8 DSR Tests Precision Results 


\section{Equipment Evaluation}

The Bohlin Dynamic Shear Rheometer required significant training time to become proficient in its use. The sample specimen preparation and operation of the rheometer allowed for potential sources of user error. Trimming the asphalt specimen required great care. The sample must consistently be trimmed such that the vertical sides of the asphalt specimen are flush with edges of the parallel plates. The edges of the asphalt specimen should slightly bulge out when the plates are set to the test gap position if the specimen was trimmed correctly. Poor trimming technique can produce test specimens with either excessive material or insufficient material resulting in erroneous tests. Heating the trimming tool is a common technique to aid in the trimming of the asphalt specimen. Heating the tool, however, requires careful attention. If the trimming tool is heated too much, the asphalt specimen will bleed and flow from between the plates while trimming, thus leaving an inadequate sized specimen for testing. Conversely, heating the trimming tool too little could cause the asphalt specimen to stick to the trimming tool, resulting in the asphalt specimen being pulled from between the plates, reducing the asphalt specimen size as well. Both scenarios would produce erroneous test results.

Setting the zero gap is a very critical procedure as well. As described in Chapter 2 , the zero gap is achieved when the gap between the upper and lower plates is reduced to the point when the plates just touch. The actual test gap obtained, $1 \mathrm{~mm}$ or $2 \mathrm{~mm}$, depending upon the type of asphalt tested, is relative to the zero gap. For example, if the zero gap is incorrectly set, such that a $20 \mu \mathrm{m}$ gap actually exists between the plates, then the actual test gap will likewise be $20 \mu \mathrm{m}$ greater than the target test gap. Given that the target gap is a first-order term in the calculation of the complex modulus, a gap error can produce erroneous test results. For example, if the target test gap is $2.00 \mathrm{~mm}$ and a $20 \mu \mathrm{m}$ gap error is experienced, then a $1 \%$ error in the complex modulus will result.

There was initial concern with the craftsmanship of the Bohlin Mechanical DSR II. The upper and lower plates could never be aligned perfectly such that the periphery of both the plates were flush. The upper and lower plates always maintained a 
fraction of a millimeter offset even after repeated attempts to align the plates. There was also concern regarding what appeared to be significant amount of play observed in the upper spindle. The upper spindle can be slightly moved horizontally when light pressure is applied by the operator's fingers. Horizontal movement of the spindle, which was exaggerated by the misalignment of the upper and lower plates, was also observed when the trimming tool was dragged along the parallel plate's periphery. There was concern that this undesired movement would adversely affect the precision of the trimming procedure, which in turn could lead to erroneous tests.

Consistent test repeatability could not be achieved during the trial testing period. Therefore, Dennis Burke of Citgo visited the lab to evaluate the equipment performance and user technique. Mr. Burke did not express concern over the spindle movement and plate misalignment. Mr. Burke recalibrated the temperature controller and overall calibration with the standard viscosity fluid.

The repeatability problems persisted during Mr. Burke's evaluation. Therefore, a service technician was contacted to check the DSR. The technician evaluated the DSR and reported that the play in the upper spindle was typical of the mechanical bearing type Bohlin dynamic shear rheometers. The technician did state that the play in the upper spindle was eliminated with the newer air bearing type rheometers. The technician concluded that the play in the upper spindle and the misalignment of the plates were minimal and would not affect the operation of the DSR. The technician checked the electronic circuitry unit and made adjustments. The DSR performance greatly improved following the Bohlin technician's service. Consistent test repeatability was finally achieved. Based on the results of the testing program, the DSR appears to be operating correctly.

\section{Effect of RTFO Sample Retrieval}

RTFO samples may be collected from the conditioning bottles by draining or scraping. Based on the data in Table 4.8 , the average $\mathrm{G}^{*} / \mathrm{sin} \delta$ value of the drained samples (Tests 1-4) and scraped samples (Tests 7 and 8) were $2.634 \mathrm{kPa}$ and $2.497 \mathrm{kPa}$, 
respectively. These results are within the precision requirements for the tests. Hence, it appears from these data that the method used to retrieve the samples from the RTFO bottles does not impact subsequent test results.

\section{Test Protocol and Specifications}

Precision and repeatability of the DSR tests are dependent on proper trimming of the asphalt specimen. The heating of the trimming tool is critical to a properly trimmed asphalt specimen. Therefore, the addition of a standard protocol for trimming tool heating to the test method would improve test consistency. The development of a standard trimming tool would be beneficial as well.

The AASHTO TP5 test standards provide single-operator precision statements for the DSR tests on original binder, RTFO-aged binder, and RTFO/PAV-aged binder. The precision statements are established for "duplicate" tests. With regard to the tests on conditioned asphalt, whether RTFO-aged or PAV-aged, the standard is silent on when the split of the asphalt material for the creating the test specimens is to be performed. The material for the test specimens could be split from the neat tank material, conditioned separately, and then tested. Or, the material for the test specimens could be split from the same container of conditioned material. The addition of a standard sequence to the test method would eliminate the existing ambiguity.

\subsection{Bending Beam Rheometer}

\section{Testing Results Analysis}

A summary of the bending beam rheometer test results is presented in Table 4.9. The results of each test are checked against SHRP performance specifications for creep stiffness, $\mathrm{S}(\mathrm{t})$, and the $\mathrm{m}$-value, $\mathrm{m}(\mathrm{t})$. All of the BBR tests passed the SHRP specification for the m-value. Tests 11, 15, and 16 each failed the SHRP specification for creep stiffness by exceeding the maximum allowable $300 \mathrm{MPa}$ limit. Test 12 was first and only test that met the SHRP specification for creep stiffness. However, the test temperature at 60 seconds was $-11.2{ }^{\circ} \mathrm{C}$, which differed from $-12{ }^{\circ} \mathrm{C}$ the target temperature by more than the allowable $\pm 0.1{ }^{\circ} \mathrm{C}$ difference, as per AASHTO TP1. The creep stiffness produced by 
Test 12 was only $246.4 \mathrm{MPa}$ while Tests 11,15 , and 16 produced creep stiffness results ranging from 301.2 MPa to 322.3 MPa. It is doubtful that a temperature difference of $+1.0{ }^{\circ} \mathrm{C}$ would result in a stiffness reduction of over $54 \mathrm{MPa}$, which is the case when comparing Test 12 results to Tests 11, 15 and 16. Therefore, the BBR results for creep stiffness are suspect.

\begin{tabular}{|c|c|c|c|c|c|c|c|c|c|}
\hline $\begin{array}{c}\text { Test } \\
\text { No. }\end{array}$ & $\begin{array}{c}\text { Temp. } \\
\left({ }^{\circ} \mathbf{C}\right)\end{array}$ & $\begin{array}{c}\text { Load, } \\
\mathbf{P} \\
(\mathbf{m N})\end{array}$ & $\begin{array}{c}\text { Deflection, } \\
\mathbf{\delta} \\
(\mathbf{m m})\end{array}$ & $\begin{array}{c}\text { Creep } \\
\text { Stiffness, } \\
\mathbf{S}(\mathbf{6 0}) \\
(\mathbf{M P a})\end{array}$ & $\begin{array}{c}\text { Max. } \\
\text { Allow. } \\
\mathbf{S}(\mathbf{6 0})^{2} \\
(\mathbf{M P a})\end{array}$ & $\begin{array}{c}\mathbf{S} \mathbf{6 0}) \\
\text { Test } \\
\text { Results }\end{array}$ & $\begin{array}{c}\text { m-value, } \\
\mathbf{m}(\mathbf{6 0})\end{array}$ & $\begin{array}{c}\text { Min. } \\
\text { Allow. } \\
\mathbf{m}(\mathbf{6 0})^{1}\end{array}$ & $\begin{array}{c}\mathbf{m}(\mathbf{6 0}) \\
\text { Test } \\
\text { Results }\end{array}$ \\
\hline 11 & -12.4 & 10.01 & 2.546 & 322.2572 & 300 & Failed & 0.311 & 0.300 & Passed \\
\hline 12 & -11.2 & 9.89 & 2.564 & 246.3924 & 300 & Passed & 0.329 & 0.300 & Passed \\
\hline 15 & -12.2 & 10.01 & 2.636 & 301.2026 & 300 & Failed & 0.308 & 0.300 & Passed \\
\hline 16 & -12.5 & 10.02 & 2.714 & 302.951 & 300 & Failed & 0.301 & 0.300 & Passed \\
\hline
\end{tabular}

${ }^{1}$ Fluid bath temperature at 60 seconds; Target test temperature $=-12{ }^{\circ} \mathrm{C}$

2 Per AASHTO MP1 Specifications

\section{Table 4.9 BBR Test Results}

A comparative precision analysis between the duplicate test results is provided in Table 4.10. The standard specification AASHTO TP5 for single-operator precision requires that the difference in $\mathrm{S}(60)$ and $\mathrm{m}(60)$ for duplicate BBR test results for a single operator in the same lab, expressed as a percent of their mean, shall not exceed 9.1 percent and 4.0 percent, respectively. The $\mathrm{S}(60)$ and $\mathrm{m}(60)$ results for duplicate Tests 11 and 12 each failed the AASHTO precision criteria. The comparisons, however, are not valid due to the test temperature problems encountered with Test 12. Duplicate Tests 15 and 16, however, passed the AASHTO precision criteria for both $\mathrm{S}(60)$ and $\mathrm{m}(60)$.

It is not certain why the BBR tests failed to meet the SHRP performance specifications; several possibilities do exist however. One issue to consider is the preconditioning procedures the asphalt specimens must undergo before testing. It is possible that errors in the RTFO or PAV conditioning would be reflected in the BBR test results. As discussed previously, the RTFO and PAV conditioning procedures are relatively easy to conduct with minimal sources of error. Furthermore, the DSR tests on PAV-aged specimens all passed the AASHTO criteria. Still, although unlikely, the possibility for 
user error with the conditioning procedures cannot be discounted. There are definitely potential sources of user error and equipment error associated with the BBR unit to consider. These sources are discussed in detail in the following section.

\begin{tabular}{|c|c|c|c|c|}
\hline $\begin{array}{c}\text { Test } \\
\text { Number }\end{array}$ & $\begin{array}{c}\text { Creep } \\
\text { Stiffness,S(60) } \\
\text { (MPa) }\end{array}$ & $\begin{array}{c}\text { Average S(60) } \\
\text { \% Difference }\end{array}$ & $\begin{array}{c}\text { Maximum } \\
\text { Allowable } \\
\text { \% Difference }\end{array}$ & Test Results \\
\hline 11 & 322.2572 & 26.68 & 9.1 & Failed \\
12 & 246.3924 & 0.58 & 9.1 & Passed \\
\hline 15 & 301.2026 & 302.951 & & \\
16 & & & & \\
\hline
\end{tabular}

\begin{tabular}{|c|c|c|c|c|}
\hline $\begin{array}{l}\text { Test } \\
\text { Number }\end{array}$ & m-value, $m(60)$ & $\begin{array}{l}\text { Average } \mathrm{m}(60) \\
\% \text { Difference }\end{array}$ & $\begin{array}{c}\text { Maximum } \\
\text { Allowable } \\
\% \text { Difference }\end{array}$ & Test Results \\
\hline $\begin{array}{l}11 \\
12\end{array}$ & $\begin{array}{l}0.311 \\
0.329\end{array}$ & 5.63 & 4 & Failed \\
\hline $\begin{array}{l}15 \\
16\end{array}$ & $\begin{array}{l}0.308 \\
0.301\end{array}$ & 2.30 & 4 & Passed \\
\hline
\end{tabular}

${ }^{1}$ Per AASHTO TP1 Precision Criteria

Table 4.10 BBR Tests Precision Results

\section{Equipment Evaluation}

The ATS bending beam rheometer operation and sample specimen preparation and handling required a significant amount of time to develop user technique. The nature of specimen beam preparation and handling provides several opportunities for user error. The calculated stiffness of the beam is proportional to the thickness, raised to the third power (Petersen, et al., 1994b). Therefore, it is critical that the prepared specimen beams be to the exact dimension required and that the shape of the beam is not distorted during demolding. Care must be taken to ensure that air pockets do not form while pouring the PAV-aged residue into the specimen molds or erroneous tests may result. The operator must ensure that the fluid bath remain clean. The specimen beams become very brittle when submerged in the BBR fluid bath, which often results in fragments being collected in the fluid bath. These fragments can collect on the beam supports and loading head and 
lead to erroneous deflection results.

Significant difficulty was experienced when trying to calibrate linear variable differential transformer (LVDT) of the BBR. The LVDT is calibrated via a factorycalibrated stepped thickness gage. The stepped thickness gage is a disk with 4 slots; each slot has a different calibrated thickness. Prior to BBR testing, a calibration verification procedure is conducted by placing the stepped thickness gage in the BBR loading frame and a thickness reading is taken at each of the 4 slots. The deviation between the measured thickness and the actual gage thickness at each slot is recorded. The total deviation is calculated and checked against the manufacturer's LVDT precision specifications. If the total thickness deviation is greater than the precision specification, a calibration procedure, that is very similar to the calibration verification, is conducted using the same stepped thickness gage.

The slots in the disk are approximately $12 \mathrm{~mm}$ wide. It was observed that the thickness varied across the each slot. It is recommended by the ATS to always take thickness readings at the same location on the disk: either at the center or the same edge of the slot. The manufacturer's direction was followed; however, the calibration of the LVDT could never be achieved. Therefore, the BBR loading unit and the stepped thickness gage were sent back to the ATS for investigation. No defect was found with the loading unit and LVDT. The stepped thickness gage, however, was observed to be out of specification and required additional milling.

The steps taken by ATS did not eliminate the problem; the LVDT calibration still could not be verified consistently. Therefore, the location of thickness readings in each slot was selected as necessary to "force" a successful LVDT calibration during testing. While this procedure is not the correct calibration methodology, it was necessary to proceed with the testing program. It is possible that the problems encountered with the BBR test results are a consequence of the LVDT calibration difficulties. 


\section{Test Protocol and Specifications}

As with the AASHTO test method for the DSR tests, the single-operator precision statement for the BBR tests is silent on when the split of the asphalt material for the creating the test specimens is to be performed. A standard sequence should be provided in the test method as well. The AASHTO test standard specification for a suitable fluid bath should be more specific. A standard fluid bath specification would be beneficial. 


\section{Chapter 5}

\section{Conclusion and Recommendations}

\section{$5.1 \quad$ Conclusions}

The objective of this research, to critically evaluate the SHRP binder testing equipment in the Asphalt Technology Laboratory at WVU and the corresponding standard test methods, was accomplished. The equipment calibration, operation, and testing procedures were evaluated and the results documented. An evaluation of the equipment precision and repeatability, as per the applicable AASHTO precision and bias statements, was conducted. Equipment problems were identified and corrected when possible. Potential sources of equipment and operator error were also identified and documented.

The Despatch rolling thin film oven, the Applied Test Systems, Inc. pressure aging vessel, and the NAPCO vacuum oven performed satisfactorily during this research. However, their performance is more difficult to assess since they only condition asphalt cements; no direct test data is produced to evaluate. The dynamic shear rheometer tests provided good results when testing both RTFO-aged and PAV-aged asphalt specimens. The bending beam rheometer test results on PAV-aged asphalt specimens were poor. However, the performance of the BBR is suspect; therefore, it is doubtful that the poor results are due to erroneous RTFO or PAV conditioning procedures.

No significant sources of equipment or operator error were identified with the pressure aging vessel or the vacuum oven. The scraping procedure for RTFO residue collection, however, proved to be problematic. As discussed in Section 4.2, the AASHTO T 240 requirement of obtaining at least $90 \%$ of the RTFO residue when scraping the RTFO bottles could never be achieved. No literature was found to document the need for the scraping requirement or the consequences for not obtaining this result. In addition, the AASHTO standards do not specify a minimum amount of RTFO residue that must be collected when draining the bottles in lieu of the scraping procedure. The 
DSR test results from this research showed no difference between the draining and scraping RTFO-residue collection techniques. If the $90 \%$ retrieval specification for scraping is maintained, a standard tool should be developed and specified in the AASHTO standards.

The Brookfield RVDV-III rotational viscometer was easy to operate with simple test sample preparation procedures. No significant sources of operator error were identified. The Brookfield RVDV-III provided good repeatability, with the exception of a few erroneous tests.

Problems were encountered with the test temperature display on the Brookfield. The test temperature displayed on the Thermosel temperature controller consistently differed from the temperature displayed on the computer monitor, as determined by the Brookfield software, by more than the allowable variance in the test method. As per the manufacturer's verbal instruction, this problem was "resolved" by ignoring the temperature displayed by the temperature controller.

The AASHTO precision and bias criteria were never achieved on several tests due to spindle selection and test speed. Furthermore, all the tests were conducted outside the measuring capabilities of the unit, again, due to the spindle selection and test speed used throughout the testing program. The error in spindle/speed selection was due, in large part, to the ambiguity that exists on the topic between the testing protocol and related reference sources (i.e. AASHTO standard specifications, Asphalt Institute publications, SHRP publications). The testing protocol should be altered with respect to spindle and test speed selection to ensure the equipment is operated in a manner consistent with the measurement capability of the unit and the precision required from the test.

Another problem noted with the AASHTO test method is the absence of test temperature criterion for conducting tests for equiviscous temperatures. Inclusion of standard test temperatures into the test method would be beneficial. 
The dynamic shear rheometer and bending beam rheometer tests were the most difficult to perform. Both the DSR and BBR tests required significant training time to develop the skills necessary for proper specimen preparation and unit operation. Proper sample preparation is critical due to the nature of the tests; therefore, user skill is a key component to the precision and repeatability of both tests.

Although there was an initial difficulty in producing repeatable test results, the Bohlin Instruments DSR II mechanical dynamic shear rheometer achieved satisfactory results following service from the manufacturer and continued to perform well throughout the testing program. The performance of the ATS bending beam rheometer, on the other hand, was poor. The BBR failed to produce acceptable results. The poor performance of the ATS BBR could be a result of the calibration difficulties experienced during the research. The linear variable differential transformer could never be properly calibrated even following service from the manufacturer. The calibration problems need to be resolved before a complete evaluation of the unit can be obtained.

The standard specifications and test protocol for the DSR and BBR were satisfactory for the most part; however, they do not provide a standard sequence for the splitting of conditioned asphalt for creating DSR and BBR test specimens. The addition of a standard sequence would be advantageous. The addition of standard trimming tool and a standard method for heating the trimming tool to the DSR testing protocol would be beneficial. The AASHTO test standard for a suitable fluid bath for the BBR should be more specific. A standard fluid bath composition should be specified.

\subsection{Recommendations}

A level of confidence in the performance of the Despatch rolling thin film oven, the Applied Test Systems, Inc. pressure aging vessel, the NAPCO vacuum oven, and the Bohlin Instruments DSR II mechanical dynamic shear rheometer was achieved by the conclusion of the research such that the units should be considered reliable and ready for continued research and industry testing. 
The evaluation of the Brookfield RVDV-III rotational viscometer should continue. Additional tests should be conducted on PG 70-22 asphalt binder, as well as other PG grades, using the appropriate spindle type and test speed such that the tests are done within the measuring capabilities of the unit and the precision requirements of the test method.

Exhaustive efforts were made during the research to bring the calibration of the Applied Test Systems, Inc. bending beam rheometer into compliance. However, calibration of the unit could never be achieved. The complete unit should be sent back to the manufacturer for their assessment of the calibration problems. A further evaluation of the Applied Test Systems, Inc. bending beam rheometer is not recommended until the calibration problems are resolved.

The shortcomings of the testing protocol, as summarized in the Conclusions section of this research, should be addressed. The suggested improvements to the test method would provide a more consistent and comprehensive protocol and eliminate ambiguity. 


\section{References}

Anderson, D. A., Christensen, D.W., Bahia, H.U., Dongre, R., Sharma, M.G., Antle, C.E., Button, J., "Binder Characterization and Evaluation; Volume 3: Physical Characterization," Strategic Highway Research Program Report No. SHRP-A-369, National Research Council, Washington, D.C., 1994.

Bahia, H.U., Anderson, D.A., "Strategic Highway Research Program Binder Rheological Parameters: Background and Comparison with Conventional Properties," Transportation Research Record 1488, TRB, National Research Council, Washington, DC, pp. 32-39, 1995 a.

Bahia, H.U., and Anderson, D.A., "The Development of the Bending Beam Rheometer; Basics and Critical Evaluation of the Rheometer," Physical Properties of Asphalt Cement Binders: ASTM STP 1241, John C. Hardin, Ed., American Society for Testing and Materials, Philadelphia, PA., pp. 28-50, 1995 b.

Bahia, H.U., and Anderson, D.A., "The Pressure Aging Vessel (PAV): A Test to Simulate Rheological Changes Due to Field Aging," Physical Properties of Asphalt Cement Binders: ASTM STP 1241, John C. Hardin, Ed., American Society for Testing and Materials, Philadelphia, PA., pp. 67-88, 1995c.

http:// www.asphaltinstitute.org/superpav/chart.jpg, accessed 9/23/2003.

Huang, Y.H., Pavement Analysis and Design, Prentice Hall, Englewood Cliffs, N.J., 1993.

"Laboratory Mixing and Compaction Temperatures," Asphalt Institute Technical Bulletin, http://www.asphaltinstitute.org/superpav/mixcompt.html, accessed 8/19/01.

Petersen, J.C., Robertson, R.E., Branthaver, J.F., Harnsberger, P.M., Duvall, J.J., Kim, S.S., Anderson, D. A., Christensen, D.W., Bahia, H.U., "Binder Characterization and Evaluation; Volume 1," Strategic Highway Research Program Report No. SHRP-A-367, National Research Council, Washington, D.C., 1994a.

Petersen, J.C., Robertson, R.E., Branthaver, J.F., Harnsberger, P.M., Duvall, J.J., Kim, S.S., Anderson, D. A., Christensen, D.W., Bahia, H.U., Dongre, R., Antle, C.E., Sharma, M.G., "Binder Characterization and Evaluation; Volume 4: Test Methods," Strategic Highway Research Program Report No. SHRP-A-370, National Research Council, Washington, D.C., 1994b. 
Roberts, F.L., Kandhal, P.S., Brown, E. Ray, Lee, D., Kennedy, T.W., Hot Mix Asphalt Materials, Mixture Design and Construction, NAPA Research and Education Foundation, Lanham, MD, 1996.

Thibodeau, L., Brookfield Engineering Laboratories, Inc., Verbal communication via telephone, 11/18/2003

Whorlow, R.W., Rheological Techniques, Ellis Horwood Limited, West Sussex, England 1992. 


\section{Vita}

Michael E. Pumphrey was born in Weston, West Virginia on November 27, 1969. He received his Bachelor of Science degree in Civil and Environmental Engineering from West Virginia University with honors in May 1998. He has since completed graduate level course work in the areas of pavement design, geotechnical and geoenvironmental engineering, and infrastructure management and has successfully passed the Fundamentals of Engineering exam.

Michael currently resides in Morgantown, West Virginia where he is employed by SAI Consulting Engineers, Inc. as a member of the highway engineering group. Michael is currently a candidate for the Masters of Science degree in Civil Engineering at West Virginia University, and plans to graduate in December, 2003. Michael also plans to take the Principles and Practice of Engineering exam in the spring of 2004. 